\title{
Oropharyngeal Neisseria gonorrhoeae
}

Citation for published version (APA):

van der Veer, B. M. J. W. (2021). Oropharyngeal Neisseria gonorrhoeae: the key for surveillance.

[Doctoral Thesis, Maastricht University]. Maastricht University. https://doi.org/10.26481/dis.20210706bv

Document status and date:

Published: 01/01/2021

DOI:

10.26481/dis.20210706bv

Document Version:

Publisher's PDF, also known as Version of record

\section{Please check the document version of this publication:}

- A submitted manuscript is the version of the article upon submission and before peer-review. There can be important differences between the submitted version and the official published version of record.

People interested in the research are advised to contact the author for the final version of the publication, or visit the DOI to the publisher's website.

- The final author version and the galley proof are versions of the publication after peer review.

- The final published version features the final layout of the paper including the volume, issue and page numbers.

Link to publication

\footnotetext{
General rights rights.

- You may freely distribute the URL identifying the publication in the public portal. please follow below link for the End User Agreement:

www.umlib.nl/taverne-license

Take down policy

If you believe that this document breaches copyright please contact us at:

repository@maastrichtuniversity.nl

providing details and we will investigate your claim.
}

Copyright and moral rights for the publications made accessible in the public portal are retained by the authors and/or other copyright owners and it is a condition of accessing publications that users recognise and abide by the legal requirements associated with these

- Users may download and print one copy of any publication from the public portal for the purpose of private study or research.

- You may not further distribute the material or use it for any profit-making activity or commercial gain

If the publication is distributed under the terms of Article $25 \mathrm{fa}$ of the Dutch Copyright Act, indicated by the "Taverne" license above, 


\section{Oropharyngeal Neisseria gonorrhoeae:

\author{
the key for surveillance
}

Brian M.J.W. van der Veer 
Oropharyngeal Neisseria gonorrhoeae: the key for surveillance Brian M.J.W. van der Veer

ISBN 9789464370331

Cover illustration by Mayk Lucchesi Layout by Brian M.J.W. van der Veer Printed by Grafipoint

(c) Copyright Brian M.J.W. van der Veer, Sambeek 2021

All rights reserved. No part of this thesis may be reproduced, stored or transmitted in any way or by any means without the prior permission of the author, or when applicable, of the publishers of the scientific papers.

Printing of this thesis was financially supported by the Netherlands Society of Medical Microbiology (NVMM), the Care and Public Health Research Institute (CAPHRI) of Maastricht University (UM) and Maastricht University Medical Centre+ (MUMC+). 


\title{
Oropharyngeal Neisseria gonorrhoeae: the key for surveillance
}

\author{
Proefschrift \\ ter verkrijging van de graad van doctor aan de Universiteit Maastricht, \\ op gezag van de Rector Magnificus, Prof. dr. Rianne M. Letschert \\ volgens het besluit van het College van Decanen, \\ in het openbaar te verdedigen \\ op dinsdag 6 juli 2021 om 13:00uur \\ door
}

Brian Marinus Johannus Wilhelmus van der Veer 
Promotor

Prof. dr. Christian J.P.A. Hoebe

Copromotores

Dr. Lieke B. van Alphen

Dr. ir. Petra F.G. Wolffs

Beoordelingscommissie

Prof. dr. Paul Savelkoul (voorzitter)

Prof. dr. Jochen Cals

Dr. Sylvia Bruisten (GGD Amsterdam)

Dr. Janneke Heijne (RIVM, Bilthoven) 



\section{Table of contents}

Chapter 1 General introduction

Chapter 2 Despite excellent test characteristics of the cobas ${ }^{\circledR} 4800$ CT/NG 25 assay, detection of oropharyngeal Chlamydia trachomatis and Neisseria gonorrhoeae remains challenging Julius M. van Niekerk, Brian M.J.W. van der Veer, Christian J.P.A. Hoebe, Jeroen van de Bovenkamp, Christel van Herk, Inge H.M. van Loo, Lieke B. van Alphen, Petra F.G. Wolffs. JCM. 2020 doi: 10.1128/JCM.02137-20

Chapter 3 Men and Women Have Similar Neisseria gonorrhoeae Bacterial Loads: a Comparison of Three Anatomical sites Brian M.J.W. van der Veer, Christian J.P.A. Hoebe, Nicole H.T.M. Duker-Muijrers, Lieke B. van Alphen, Petra F.G. Wolffs. JCM. 2020 doi: 10.1128/JCM.01171-20

Chapter 4 Culture-free genotyping of Neisseria gonorrhoeae revealed distinct strains at different anatomical sites in a quarter of patients, the Netherlands, 2012 to 2016 Brian M.J.W. van der Veer, Petra F.G. Wolffs, Christian J.P.A. Hoebe, Nicole H.T.M. Duker-Muijrers, Lieke B. van Alphen. Eurosurveillance. 2018 doi: 10.2807/15607917.ES.2018.23.50.1800253

Chapter 5 Metagenomic analysis shows distinct Neisseria gonorrhoeae strains at separate anatomical sites occur more commonly than mixed strain infections: implications for surveillance. Brian M.J.W. van der Veer, Petra F.G. Wolffs, Christian J.P.A. Hoebe, Nicole H.T.M. Duker-Muijrers, Lieke B. van Alphen. Submitted 
Chapter 6 Culture-free Genotyping Improves Surveillance of Neisseria gonorrhoeae, Especially in Oropharyngeal Samples, the Netherlands, 2017 to 2018.

Michiel H.C. Slaats, Brian M.J.W. van der Veer, Lieke B. van Alphen,

Christian J.P.A. Hoebe, Nicole H.T.M. Dukers-Muijrers, Petra F.G. Wolffs.

Submitted

Chapter 7 Potential Neisseria gonorrhoeae outbreak in young heterosexuals $(<25)$; culture-independent genotyping revealed three clusters, the Netherlands, October 2017 to March 2019

Brian M.J.W. van der Veer, Petra F.G. Wolffs, Christian J.P.A. Hoebe,

Juliën N.A.P. Wijers, Gènevieve A.F.S. van Liere, Marita I.L.S. Werner, Amanja Verhaegh, Nicole H.T.M. Duker-Muijrers, Lieke B. van Alphen.

STD. 2021 doi: 10.1097/OLQ.00000000000001370

Chapter 8 Despite no ceftriaxone resistance in the Netherlands many Neisseria gonorrhoeae isolates have a mosaic penA gene Brian M.J.W. van der Veer, Christian J.P.A. Hoebe, Nicole H.T.M. Duker-Muijrers, Lieke B. van Alphen, Petra F.G. Wolffs. Manuscript in preparation

Chapter 9 Summary and general discussion

$\begin{array}{lll}\text { Addendum } & \text { Samenvatting en algemene discussie } & 176\end{array}$ Impact paragraph 188 List of publications 192

Curriculum vitae 194

Dankwoord 196 


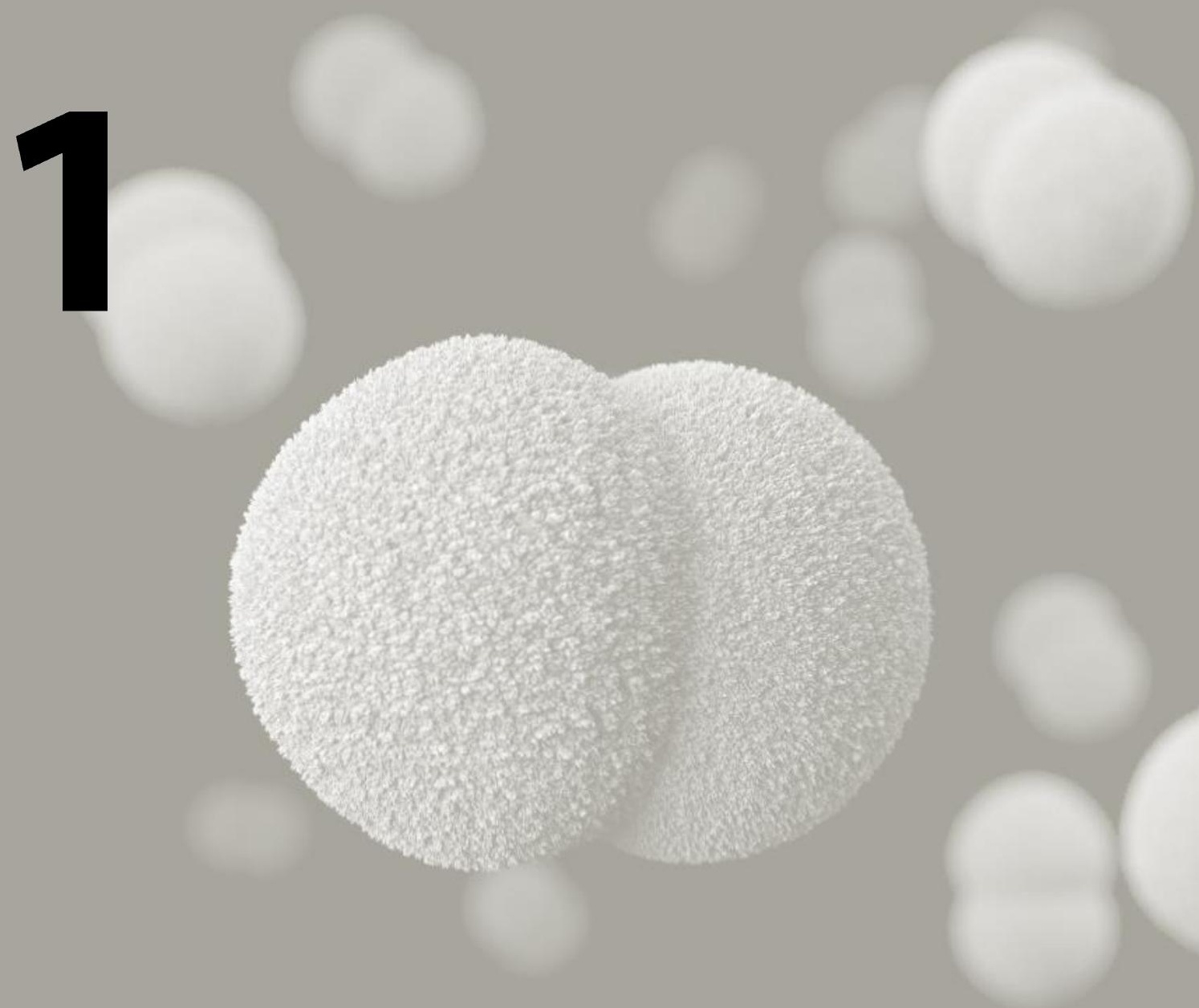


General introduction 


\section{Background}

Sexually transmitted infections (STI) are a major global public health challenge [1]. The four most common curable bacterial STls are Chlamydia trachomatis, Neisseria gonorrhoeae, Trichomonas vaginalis, and Treponema pallidum. The World Health Organization (WHO) estimated that 376 million infections of these four STI occur each year with $N$. gonorrhoeae (gonorrhea) being the second most common bacterial STI with an estimated global incidence of 64 million new cases annually [2]. Gonorrhea can infect the urogenital tract in both women and men but also the mucosa of extra-genital sites such as the rectum and oropharynx [3]. Another route of transmission is from mother to child during birth that causes an ocular infection.

Many gonorrhea infections remain hidden to care because of stigma and their asymptomatic nature, especially in women [4-7]. Therefore, these infections may remain undiagnosed and untreated that can lead to ongoing transmission and complications [8]. In women, gonorrhea can cause pelvic inflammatory disease, infertility, ectopic pregnancy, and maternal death [4]. In men, urethral infections are frequently symptomatic and can cause urethritis with pus-like discharge, dysuria, and pain or swelling in one testicle [9]. In addition, infection with gonorrhea has been associated with an increased risk of transmission and acquisition of human immunodeficiency virus (HIV) $[3,10]$.

\section{Epidemiology}

Gonorrhea is the second most common bacterial STI worldwide and this is also the case in the Netherlands $[2,11]$. There were 7,362 gonorrhea cases diagnosed at an STI clinic and an estimated 9,500 cases in general practice in the Netherlands in 2018 [11]. The reported positivity rate in STI clinics was $1.6 \%$ in women, $1.8 \%$ in heterosexual men, and $11.0 \%$ in men who have sex with men (MSM). Figure 1 shows the positivity rate and number of tests in STI clinics from 2009-2018. The positivity rates of these groups remained stable since 2015 because triage and number of tests has not changed. This is because of the implementation of the latest Dutch STI guidelines and budget cap 
(ASG-regeling) in 2015 [12]. In the guidelines several sexual risk-groups for gonorrhea infection acquisition are described such as MSM, sex workers, and young people [4, 12].

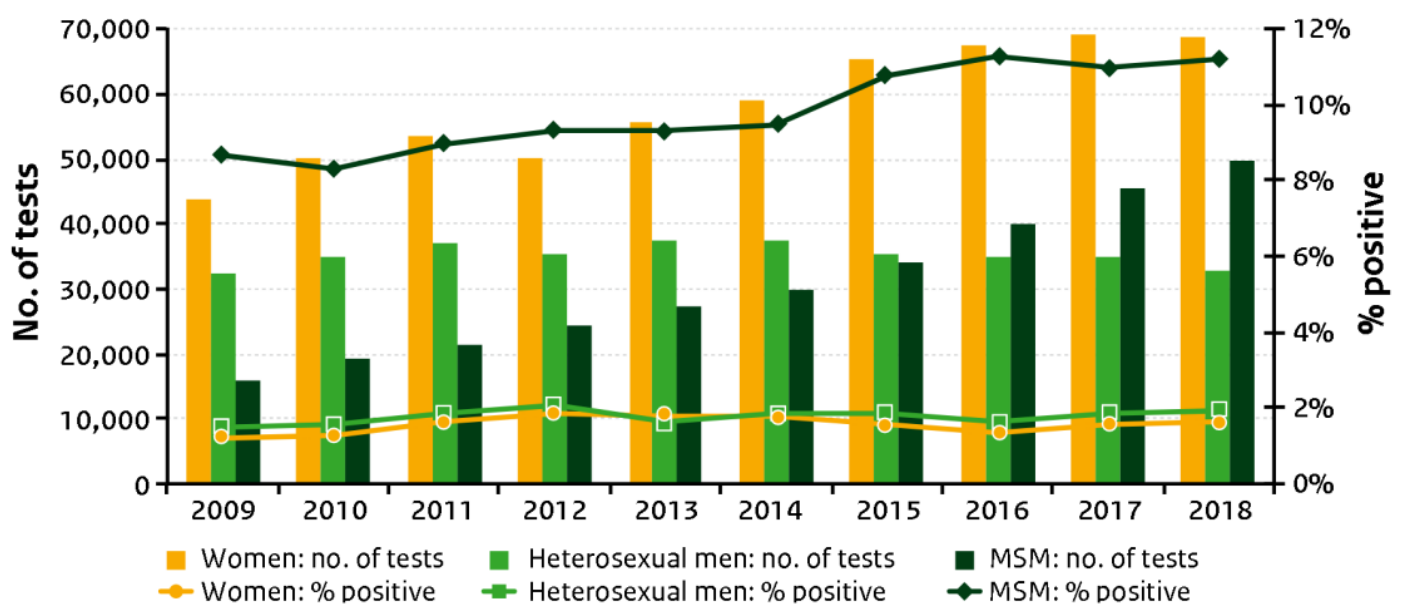

Figure 1. Total number of tests and positivity rate of gonorrhea by sexual risk-group, 20092018. Source: Slurink et al. 2019. Sexually transmitted infections in the Netherlands 2018. National Institute for Public Health and Environment.

\section{Biology of Neisseria gonorrhoeae}

Neisseria gonorrhoeae is a Gram-negative diplococcus that is part of the genus Neisseria of which most species are commensal and colonize the nasopharynx [3]. These Neisseria species are a substantial part of the human nasal and oropharyngeal microbiome. Other Neisseria species colonize a range of other hosts such as cats and dogs [13]. Neisseria gonorrhoeae and $N$. meningitidis are the two pathogenic species, with the second causing bacterial meningitis [14]. As both commensal and pathogenic Neisseria species share the same niches they compete in colonization and nutrition [3].

As gonorrhea is an obligate human pathogen it cannot survive outside the host [3]. Therefore, transmission relies on unprotected sexual intercourse [15]. Gonorrhea is easily transmitted from men to women as it attaches to sperm and high bacterial load is observed in ejaculates [16-18]. However, the transmission efficiency from women to men is less clear $[3,19]$. This might be because gonorrhea must be desialylated to infect the male urinal tract but gonorrhea is sialylated during infection in women [20]. After transmission, gonorrhea needs to adhere to the mucosal epithelium, in which type IV pili 
and opacity (Opa) proteins are essential in both women and men [3]. Type IV pili is responsible for the initial adherence and micro-colony formation but is also involved in the natural competence and immune evasion [21-23]. Opa proteins mediate adherence by multigene phase variation that also results in antigenic variation to avoid the immune system $[24,25]$. Another mechanism to avoid the immune system is binding to components of the complement system ( $\mathrm{C} 4 \mathrm{BP}$ and factor $\mathrm{H}$ ) by porB to suppress the neutrophil response [26]. This suppresses the inflammatory response and result in an asymptomatic infection [3]. However, the modulation of the immune system is still under debate as gonorrhea can also deliberately activate and attract neutrophils and macrophages that might facilitate transmission [3, 27].

\section{Diagnoses and treatment}

Gonorrhea can be cultured on agar plates like chocolate agar medium and was considered the golden standard $[15,28]$. Culture of gonorrhea is inexpensive to perform but is not ideal for routine diagnostics because of the demanding nutritional and environmental conditions. These issues also lead to frequent failure of gonorrhea culture as it requires viable gonorrhea after transport to the laboratory. Still, culture is performed to date but mainly for surveillance of antimicrobial resistance (AMR), in which culture remains to be considered as the golden standard [28, 29].

In modern routine diagnostics Nucleic Acid Amplification Testing (NAAT) is performed to detect gonorrhea RNA or DNA $[11,15,30]$. These NAATs are the recommended tests as they outperform culture in sensitivity, do not require viable gonorrhea to be present in the sample, and can be automated but have a lower specificity compared to culture [9, 15]. The first generation of NAATs for gonorrhea diagnostics presented issues with falsepositive results by detection of commensal Neisseria species in a sample that was negative for gonorrhea [31]. As a direct consequence none of the first generation NAATs are approved for extra-genital (anorectal and oropharyngeal) testing as commensal Neisseria species are commonly present at those anatomical sites $[28,31]$. The second generation of NAATs vastly improved on this aspect but still almost all NAATs are not 
officially approved for extra-genital testing. Nevertheless, in practice, NAATs are widely used for extra-genital testing nowadays.

Uncomplicated gonorrhea is treated with a single dose of $500 \mathrm{mg}$ ceftriaxone intramuscular in the Netherlands [12]. Resistance of gonorrhea towards the last first-line treatment of ceftriaxone has been reported by different countries worldwide but not in the Netherlands [11, 32]. Therefore, international guidelines recommended a dualtherapy of $500 \mathrm{mg}$ ceftriaxone with $1 \mathrm{~g}$ of azithromycin to slow down AMR in gonorrhea $[4,30]$. However, gonorrhea developed azithromycin resistance rapidly thereby limiting the dual-therapy strategy $[11,33,34]$. In addition, the susceptibility of gonorrhea towards ceftriaxone is decreasing. Therefore, other antibiotics with adverse events like gentamycin have to be considered but also novel antimicrobials should be developed $[32,35]$.

\section{Prevention and screening}

Identification and effective treatment of patients infected with gonorrhea can interrupt onward transmission and development of serious complications but many gonorrhea infections are asymptomatic and therefore the patient may not actively seek care $[4,5$, 7]. This lead to several control strategies to reduce gonorrhea prevalence and prevent AMR: (I) education and counseling on safer sexual practices, (II) identification of both asymptomatic and symptomatic patients, (III) evaluation, treatment and counseling of sex partners, (IV) effective diagnosis and treatment, and (V) gonococcal resistance surveillance and stewardship [4, 36].

Since 2015 a budget cap and triage (ASG-regeling) was implemented by the Dutch government [12]. The triage is based on research in which populations at risk to acquire gonorrhea infections were identified. Examples are MSM, sex workers, individuals notified for an STI, and young people $(<25)$ for which free of charge testing is provided at an STI clinic. Furthermore, testing is recommended for individuals presenting with symptoms related to gonorrhea like vaginal discharge in women and urethritis in men. Patients are tested urogenitally for gonorrhea at each STI clinic consultation but based on sexual risk group and risk factors additional extra-genital samples are tested. The STI 
clinic guideline covers different test policies for women, heterosexual men, and MSM. MSM are routinely and systematically tested at both anorectal and oropharyngeal sites (extra-genital). Heterosexual patients are only tested extra-genitally when reporting symptoms, anal or oral sexual behavior in the last six months. However, the need for extra-genital testing and clinical relevance of these infections in heterosexuals is under debate $[37,38]$. One of the key questions is whether oropharyngeal gonorrhea has sufficient transmission potential to infect others as it is believed that the oropharynx is a reservoir for AMR development for gonorrhea [32, 39, 40]. A possible measure for transmission potential is the load of gonorrhea as this has been described in other STIS [41-43].

\section{Oropharyngeal gonorrhea}

Recent epidemiological studies demonstrate that oropharyngeal NG is common in MSM and women $[44,45]$. Prevalence of oropharyngeal NG was $5.5 \%$ in MSM and $1.4 \%$ in women when routine universal testing was performed and shows that half of the oropharyngeal NG infections were oropharyngeal-only infections. As oropharyngeal gonorrhea infections are common, frequently asymptomatic, and are linked to AMR development it is a very important anatomical site of infection [32, 44-47]. The oropharyngeal site is potentially a reservoir for resistance development as commensal Neisseria species are commonly present that can exchange DNA with gonorrhea [32, 47]. In addition, kissing, oral sex, and saliva use as lubricant are common sexual practices in both heterosexuals and MSM [38, 46, 48]. This can result in unnoticed onward transmission and facilitate the development of multi-drug resistant gonorrhea strains. $A$ key problem for surveillance of oropharyngeal gonorrhea is that infections of this particular anatomical site are difficult to culture and thus limits characterization of strains $[11,28]$. However, the exact role of oropharyngeal gonorrhea infections is unknown. The transmission potential of oropharyngeal gonorrhea might be lower compared to genital and urethral infections in MSM but the published studies are relatively small and no data exists of women and heterosexual men [39, 40,49]. It is also unknown if resistance development in the oropharynx is spontaneous or rare with clonal spread of resistant strains [32]. 


\section{Antimicrobial resistance}

During the pre-antimicrobial era, treatment of gonorrhea was not quick and easy. Treatment involved having a healthier lifestyle with abstinence of sexual activity and alcohol but also urethral irrigation and hyperthermia could be used [32]. Initially, when antibiotics were discovered and applied to treat gonorrhea, most gonorrhea cases were cured with sulfonamides [50]. However, resistance towards sulfonamides developed rapidly, leading to discontinued use as a first-line treatment [50, 51]. Many other classes of antibiotics were developed and initially effective to treat gonorrhea infections but AMR was developed for all classes of antibiotics (figure 2) [32]. This limits the options for treatment of gonorrhea that could become untreatable with current antibiotics [32, 52]. To complicate things further, insufficient new antibiotics are in development thus preventing gonorrhea to become untreatable is imperative [4, 53]. Gonorrhea has developed resistance via various AMR mechanisms like enzymatic destruction or increased export of antimicrobials [32]. A very important mechanism is target alteration by creating a mosaic gene variant of penA, which encodes for the protein penicillinbinding protein-2 (PBP2), leading to ceftriaxone resistance [54-56]. As ceftriaxone is the final empirical first-line treatment and used worldwide, it is imperative to understand the development of mosaic penA genes. A likely cause is via horizontal-gene-transfer in the oropharynx as Neisseria species are naturally competent and commensal Neisseria species are commonly present in this anatomical site [57-60]. Therefore, oropharyngeal gonorrhea infections are considered as a reservoir for AMR development but it is unknown whether mosaic genes are spontaneously formed in vivo [32]. 


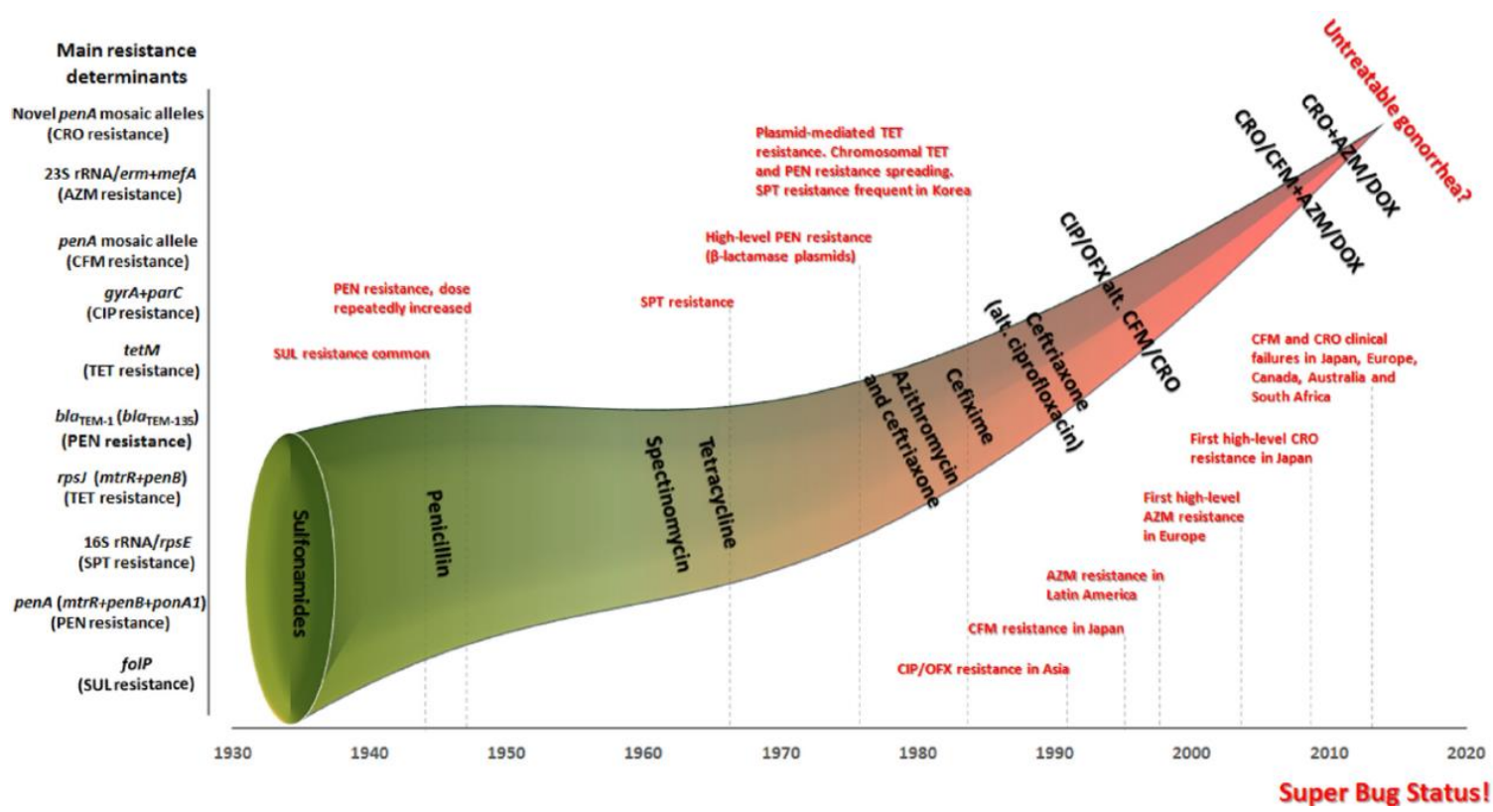

Figure 2. Historical overview of recommended antimicrobials and resistance in $\mathbf{N}$. gonorrhea. Source: Unemo et al. 2014. Antimicrobial Resistance in Neisseria gonorrhoeae in the 21st Century: Past, Evolution, and Future. Clinical Microbial Reviews.

\section{Surveillance of gonorrhea}

As gonorrhea is frequently asymptomatic and could become untreatable it is essential to implement surveillance to monitor transmission and dissemination of resistant strains [4, 61]. This lead to national and international Gonoccocal Antimicrobial Surveillance Programs (GASP) [4, 62]. In the Netherlands this program is called Gonokokken Resistentie tegen Antibiotica Surveillance (GRAS) [11]. In principle, the surveillance programs aim to gain insight in the occurrence of AMR in gonorrhea by characterizing many cultured strains. The surveillance programs should monitor trends in resistance to react to emerging resistant strains. The cultured strains in surveillance are characterized by using techniques like Multi-Locus Sequence Typing (MLST), Neisseria Gonorrhea Multi-Antigen Sequence Typing (NG-MAST), and Whole-Genome Sequencing (WGS) [6365]. MLST has the lowest discriminatory power, then NG-MAST, and WGS has the highest but is also the most expensive one. A major issue in surveillance is that all techniques require a cultured isolate but culture is known to frequently fail $[11,66]$. Also, typically only a single colony is characterized thus missing potential mixed gonococcal infections 
$[62,67]$. These infections do occur and could have an impact on the treatment of the infection as the uncultured strain could be resistant [68-70]. In addition, if mixed gonococcal infections are not considered, the transmission network is not well characterized, thereby limiting opportunities for interventions.

\section{Aim of thesis}

The aim of this thesis is to provide insight in the clinical relevance of extra-genital gonorrhea infections, particularly oropharyngeal infections, and surveillance of gonorrhea. To assess the clinical relevance of extra-genital infections we validated the Cobas 4800 CT/NG test for oropharyngeal samples and determined the bacterial load at different anatomical sites in different sexual risk groups. For surveillance of gonorrhea we developed various culture-independent methods to assess the unculturable and mixed gonococcal infections. Furthermore, nanopore-sequencing was performed to gain insight in the oral Neisseria resistome with a focus on the penA gene.

In chapter 2 we validated the cobas 4800 CT/NG test for oropharyngeal samples and provide insight in potentially missed infections.

Chapter 3 evaluates the clinical relevance of (extra) genital gonorrhea infections in both women and men by assessing the bacterial load. The bacterial load of different anatomical sites was compared and also related to sex and symptoms.

In chapter 4 we technically validated the culture-free NG-MAST method that is a cultureindependent variant of NG-MAST. This method was also used to gain insight in the extent of patients infected with different gonococcal strains at separate anatomical sites.

Chapter 5 describes the occurrence of mixed gonococcal infections in a selected group of MSM by combining culture-free NG-MAST with next-generation sequencing. The data of this chapter and chapter $\mathbf{4}$ were evaluated to review the relevance of multiple NG isolates in a patient, either mixed or at separate anatomical sites.

In chapter 6, the added value of culture-independent surveillance was tested by comparing culture-positive and culture-negative samples subjected to culture. Furthermore, the implications for current culture-dependent surveillance are given per sample material. 
A potential gonococcal outbreak in young heterosexuals in South-Limburg, the Netherlands was characterized in chapter 7. We applied culture-free NG-MAST to genotype the gonococcal strains to identify outbreak clusters. Furthermore, we describe determinants associated with these clusters, the added value of culture-independent genotyping, and discuss challenges associated with gonococcal outbreak detection and management.

Chapter 8, the oropharyngeal Neisseria resistome is assessed by characterizing penA genes and their flanking regions with nanopore-sequencing. Despite ceftriaxone resistance in gonorrhea does not exist in the Netherlands, mosaic penA genes were observed in gonorrhea. These mosaic genes were compared with commensal Neisseria species in the sample to test the possibility of recombination during the infection.

Lastly, in chapter 9, the main findings are discussed of the presented work and directions for future research are given. 


\section{References}

1. Unemo M, Bradshaw CS, Hocking JS, de Vries HJC, Francis SC, Mabey D, et al. Sexually transmitted infections: challenges ahead. Lancet Infect Dis. 2017;17(8):e235-e79.

2. Rowley J, Vander Hoorn S, Korenromp E, Low N, Unemo M, Abu-Raddad LJ, et al. Chlamydia, gonorrhoea, trichomoniasis and syphilis: global prevalence and incidence estimates, 2016. B World Health Organ. 2019;97(8):548-+.

3. Quillin SJ, Seifert HS. Neisseria gonorrhoeae host adaptation and pathogenesis. Nat Rev Microbiol. 2018.

4. World Health Organisation. Global action plan to control the spread and impact of antimicrobial resistance in Neisseria gonorrhoeae. 2012.

5. Centers for Disease Control and Prevention. Sexually Transmitted Disease Surveillance 2014. 2014.

6. Reed JL, Huppert JS, Gillespie GL, Taylor RG, Holland CK, Alessandrini EA, et al. Adolescent patient preferences surrounding partner notification and treatment for sexually transmitted infections. Acad Emerg Med. 2015;22(1):61-6.

7. Theunissen KA, Bos AE, Hoebe CJ, Kok G, Vluggen S, Crutzen R, et al. Chlamydia trachomatis testing among young people: what is the role of stigma? BMC Public Health. 2015;15:651.

8. Markle W, Conti T, Kad M. Sexually Transmitted Diseases. Primary Care. 2013;40(3):557-+.

9. Bignell C, Unemo M, European STIGEB. 2012 European guideline on the diagnosis and treatment of gonorrhoea in adults. Int J STD AIDS. 2013;24(2):85-92.

10. Ward H, Ronn M. Contribution of sexually transmitted infections to the sexual transmission of HIV. Curr Opin Hiv Aids. 2010;5(4):305-10.

11. Slurink IAL vAF, Heijne JCM, Op de Coul ELM, van Wees DA, Hoenderboom BM, Visser M, den Daas C, Woestenberg PJ, Götz HM, Nielen M, van Sighem Al, van Benthem BHB. Sexually transmitted infections in the Netherlands in 2018. National Institute for Public Health and Environment. 2019.

12. National Institute for Public Health and the Environment. Draaiboek Consult seksuele gezondheid. 2018.

13. Liu GY, Tang CM, Exley RM. Non-pathogenic Neisseria: members of an abundant, multi-habitat, diverse genus. Microbiol-Sgm. 2015;161:1297-312.

14. Hoffman O, Weber RJ. Pathophysiology and treatment of bacterial meningitis. Ther Adv Neurol Disord. 2009;2(6):1-7.

15. Centers for Disease Control and Prevention. Recommendations for the laboratory-based detection of Chlamydia trachomatis and Neisseria gonorrhoeae--2014. MMWR Recomm Rep. 2014;63(RR-02):119.

16. James-Holmquest AN, Swanson J, Buchanan TM, Wende RD, Williams RP. Differential attachment by piliated and nonpiliated Neisseria gonorrhoeae to human sperm. Infect Immun. 1974;9(5):897-902.

17. Harvey HA, Porat N, Campbell CA, Jennings M, Gibson BW, Phillips NJ, et al. Gonococcal lipooligosaccharide is a ligand for the asialoglycoprotein receptor on human sperm. Mol Microbiol. 2000;36(5):1059-70.

18. Isbey SF, Alcorn TM, Davis RH, Haizlip J, Leone PA, Cohen MS. Characterisation of Neisseria gonorrhoeae in semen during urethral infection in men. Genitourin Med. 1997;73(5):378-82.

19. Hooper RR, Reynolds GH, Jones OG, Zaidi A, Wiesner PJ, Latimer KP, et al. Cohort study of venereal disease. I: the risk of gonorrhea transmission from infected women to men. Am J Epidemiol. 1978;108(2):136-44.

20. Ketterer MR, Rice PA, Gulati S, Kiel S, Byerly L, Fortenberry JD, et al. Desialylation of Neisseria gonorrhoeae Lipooligosaccharide by Cervicovaginal Microbiome Sialidases: The Potential for Enhancing Infectivity in Men. J Infect Dis. 2016;214(11):1621-8.

21. Higashi DL, Lee SW, Snyder A, Weyand NJ, Bakke A, So M. Dynamics of Neisseria gonorrhoeae attachment: microcolony development, cortical plaque formation, and cytoprotection. Infect Immun. 2007;75(10):4743-53.

22. Craig L, Pique ME, Tainer JA. Type IV pilus structure and bacterial pathogenicity. Nat Rev Microbiol. 2004;2(5):363-78.

23. Obergfell KP, Seifert HS. The Pilin N-terminal Domain Maintains Neisseria gonorrhoeae Transformation Competence during Pilus Phase Variation. PLoS Genet. 2016;12(5):e1006069. 
24. Lambden PR, Heckels JE, James LT, Watt PJ. Variations in surface protein composition associated with virulence properties in opacity types of Neisseria gonorrhoeae. J Gen Microbiol. 1979;114(2):305-12.

25. Stern A, Brown M, Nickel P, Meyer TF. Opacity genes in Neisseria gonorrhoeae: control of phase and antigenic variation. Cell. 1986;47(1):61-71.

26. Wetzler LM, Blake MS, Barry K, Gotschlich EC. Gonococcal porin vaccine evaluation: comparison of Por proteosomes, liposomes, and blebs isolated from $\mathrm{rmp}$ deletion mutants. J Infect Dis. 1992;166(3):551-5.

27. Criss AK, Seifert HS. A bacterial siren song: intimate interactions between Neisseria and neutrophils. Nat Rev Microbiol. 2012;10(3):178-90.

28. Unemo M, Seifert HS, Hook EW, 3rd, Hawkes S, Ndowa F, Dillon JR. Gonorrhoea. Nat Rev Dis Primers. 2019;5(1):79.

29. Dillon JA. Sustainable antimicrobial surveillance programs essential for controlling Neisseria gonorrhoeae superbug. Sex Transm Dis. 2011;38(10):899-901.

30. World Health Organisation. WHO guidelines for the Treatment of Neisseria gonorrhoeae. 2016.

31. Tabrizi SN, Unemo M, Limnios AE, Hogan TR, Hjelmevoll SO, Garland SM, et al. Evaluation of six commercial nucleic acid amplification tests for detection of Neisseria gonorrhoeae and other Neisseria species. J Clin Microbiol. 2011;49(10):3610-5.

32. Unemo M, Shafer WM. Antimicrobial resistance in Neisseria gonorrhoeae in the 21st century: past, evolution, and future. Clin Microbiol Rev. 2014;27(3):587-613.

33. Starnino S, Group G-LW, Galarza P, Carvallo ME, Benzaken AS, Ballesteros AM, et al. Retrospective analysis of antimicrobial susceptibility trends (2000-2009) in Neisseria gonorrhoeae isolates from countries in Latin America and the Caribbean shows evolving resistance to ciprofloxacin, azithromycin and decreased susceptibility to ceftriaxone. Sex Transm Dis. 2012;39(10):813-21.

34. Cole MJ, Unemo M, Hoffmann S, Chisholm SA, Ison CA, van de Laar MJ. The European gonococcal antimicrobial surveillance programme, 2009. Euro Surveill. 2011;16(42).

35. Kirkcaldy RD, Weinstock HS, Moore PC, Philip SS, Wiesenfeld HC, Papp JR, et al. The efficacy and safety of gentamicin plus azithromycin and gemifloxacin plus azithromycin as treatment of uncomplicated gonorrhea. Clin Infect Dis. 2014;59(8):1083-91.

36. World Health Organisation. Global health sector strategy on sexually transmitted infections: 20162021. 2016.

37. Dukers-Muijrers NH, Schachter J, van Liere GA, Wolffs PF, Hoebe CJ. What is needed to guide testing for anorectal and pharyngeal Chlamydia trachomatis and Neisseria gonorrhoeae in women and men? Evidence and opinion. BMC Infect Dis. 2015;15:533.

38. Garner AL, Schembri G, Cullen T, Lee V. Should we screen heterosexuals for extra-genital chlamydial and gonococcal infections? Int J STD AIDS. 2015;26(7):462-6.

39. Bissessor M, Tabrizi SN, Fairley CK, Danielewski J, Whitton B, Bird S, et al. Differing Neisseria gonorrhoeae bacterial loads in the pharynx and rectum in men who have sex with men: implications for gonococcal detection, transmission, and control. J Clin Microbiol. 2011;49(12):4304-6.

40. Priest D, Ong JJ, Chow EP, Tabrizi S, Phillips S, Bissessor M, et al. Neisseria gonorrhoeae DNA bacterial load in men with symptomatic and asymptomatic gonococcal urethritis. Sex Transm Infect. 2017.

41. Vodstrcil LA, Mclver R, Huston WM, Tabrizi SN, Timms P, Hocking JS. The Epidemiology of Chlamydia trachomatis Organism Load During Genital Infection: A Systematic Review. J Infect Dis. 2015;211(10):1628-45.

42. Pedraza MA, del Romero J, Roldan F, Garcia S, Ayerbe MC, Noriega AR, et al. Heterosexual transmission of HIV-1 is associated with high plasma viral load levels and a positive viral isolation in the infected partner. J Acquir Immune Defic Syndr. 1999;21(2):120-5.

43. Lavreys L, Baeten JM, Chohan V, McClelland RS, Hassan WM, Richardson BA, et al. Higher set point plasma viral load and more-severe acute HIV type 1 (HIV-1) illness predict mortality among high-risk HIV-1-infected African women. Clin Infect Dis. 2006;42(9):1333-9.

44. van Liere G, Dukers-Muijrers N, Kuizenga-Wessel S, Gotz HM, Hoebe C. What Is the Optimal Testing Strategy for Oropharyngeal Neisseria gonorrhoeae in Men Who Have Sex With Men? Comparing Selective Testing Versus Routine Universal Testing From Dutch Sexually Transmitted Infection Clinic Data (2008-2017). Clin Infect Dis. 2020;71(4):944-51. 
45. Van Liere GAFS D-MN, Kuizenga Wessel S, Wolffs PF, Hoebe CJPA. Routine universal testing versus selective or incidental testing for oropharyngeal Neisseria gonorrhoeae in women in the Netherlands: a retrospective cohort study. Lancet Infect Dis. 2020 in press.

46. Fairley CK, Cornelisse VJ, Hocking JS, Chow EPF. Models of gonorrhoea transmission from the mouth and saliva. Lancet Infectious Diseases. 2019;19(10):E360-E6.

47. Tapsall JW, Ndowa F, Lewis DA, Unemo M. Meeting the public health challenge of multidrug- and extensively drug-resistant Neisseria gonorrhoeae. Expert Rev Anti Infect Ther. 2009;7(7):821-34.

48. Chow EPF, Cornelisse VJ, Williamson DA, Priest D, Hocking JS, Bradshaw CS, et al. Kissing may be an important and neglected risk factor for oropharyngeal gonorrhoea: a cross-sectional study in men who have sex with men. Sex Transm Infect. 2019;95(7):516-21.

49. Chow EP, Tabrizi SN, Phillips S, Lee D, Bradshaw CS, Chen MY, et al. Neisseria gonorrhoeae Bacterial DNA Load in the Pharynges and Saliva of Men Who Have Sex with Men. J Clin Microbiol. 2016;54(10):2485-90.

50. Kampmeier RH. Introduction of sulfonamide therapy for gonorrhea. Sex Transm Dis. 1983;10(2):81-4.

51. Dunlop EM. Gonorrhoea and the sulphonamides. Br J Vener Dis. 1949;25(2):81-3.

52. Eyre DW, Sanderson ND, Lord E, Regisford-Reimmer N, Chau K, Barker L, et al. Gonorrhoea treatment failure caused by a Neisseria gonorrhoeae strain with combined ceftriaxone and highlevel azithromycin resistance, England, February 2018. Euro Surveill. 2018;23(27).

53. World Health Organisation. 2019 Antibacterial agents in clinical development. 2019.

54. Lindberg $R$, Fredlund $H$, Nicholas $R$, Unemo $M$. Neisseria gonorrhoeae isolates with reduced susceptibility to cefixime and ceftriaxone: association with genetic polymorphisms in penA, $\mathrm{mtrR}$, porB1b, and ponA. Antimicrob Agents Chemother. 2007;51(6):2117-22.

55. Ohnishi M, Golparian D, Shimuta K, Saika T, Hoshina S, Iwasaku K, et al. Is Neisseria gonorrhoeae initiating a future era of untreatable gonorrhea?: detailed characterization of the first strain with high-level resistance to ceftriaxone. Antimicrob Agents Chemother. 2011;55(7):3538-45.

56. Unemo M, Golparian D, Nicholas R, Ohnishi M, Gallay A, Sednaoui P. High-level cefixime- and ceftriaxone-resistant Neisseria gonorrhoeae in France: novel penA mosaic allele in a successful international clone causes treatment failure. Antimicrob Agents Chemother. 2012;56(3):1273-80.

57. Furuya R, Onoye $Y$, Kanayama A, Saika T, lyoda T, Tatewaki M, et al. Antimicrobial resistance in clinical isolates of Neisseria subflava from the oral cavities of a Japanese population. J Infect Chemother. 2007;13(5):302-4.

58. Ameyama S, Onodera S, Takahata M, Minami S, Maki N, Endo K, et al. Mosaic-like structure of penicillin-binding protein 2 Gene (penA) in clinical isolates of Neisseria gonorrhoeae with reduced susceptibility to cefixime. Antimicrob Agents Chemother. 2002;46(12):3744-9.

59. Ito M, Deguchi T, Mizutani KS, Yasuda M, Yokoi S, Ito S, et al. Emergence and spread of Neisseria gonorrhoeae clinical isolates harboring mosaic-like structure of penicillin-binding protein 2 in Central Japan. Antimicrob Agents Chemother. 2005;49(1):137-43.

60. Osaka K, Takakura T, Narukawa K, Takahata M, Endo K, Kiyota H, et al. Analysis of amino acid sequences of penicillin-binding protein 2 in clinical isolates of Neisseria gonorrhoeae with reduced susceptibility to cefixime and ceftriaxone. J Infect Chemother. 2008;14(3):195-203.

61. Goire N, Lahra MM, Chen M, Donovan B, Fairley CK, Guy R, et al. Molecular approaches to enhance surveillance of gonococcal antimicrobial resistance. Nat Rev Microbiol. 2014;12(3):223-9.

62. European Centre for Disease Prevention and Control (ECDC). Gonococcal antimicrobial susceptibility surveillance in Europe. 2015.

63. Carannante A, Ghisetti V, Dal Conte I, Gregori G, Stella ML, Vacca P, et al. Molecular characterization of Neisseria gonorrhoeae on non-cultured specimens from multiple anatomic sites. Ann Ist Super Sanita. 2017;53(3):213-7.

64. Martin IM, Ison CA, Aanensen DM, Fenton KA, Spratt BG. Rapid sequence-based identification of gonococcal transmission clusters in a large metropolitan area. J Infect Dis. 2004;189(8):1497-505.

65. Unemo M, Dillon JA. Review and international recommendation of methods for typing neisseria gonorrhoeae isolates and their implications for improved knowledge of gonococcal epidemiology, treatment, and biology. Clin Microbiol Rev. 2011;24(3):447-58.

66. Centers for Disease Control and Prevention. Recommendations for the Laboratory-Based Detection of Chlamydia trachomatis and Neisseria gonorrhoeae - 2014. 2014.

67. Workowski KA, Bolan GA. Sexually transmitted diseases treatment guidelines, 2015. MMWR Recomm Rep. 2015;64(RR-03):1-137. 
68. Martin IM, Ison CA. Detection of mixed infection of Neisseria gonorrhoeae. Sex Transm Infect. 2003;79(1):56-8.

69. Trembizki E, Doyle C, Buckley C, Jennison A, Smith H, Bates J, et al. Estimating the prevalence of mixed-type gonococcal infections in Queensland, Australia. Sex Health. 2015;12(5):439-44.

70. Goire N, Kundu R, Trembizki E, Buckley C, Hogan TR, Lewis DA, et al. Mixed gonococcal infections in a high-risk population, Sydney, Australia 2015: implications for antimicrobial resistance surveillance? J Antimicrob Chemother. 2017;72(2):407-9. 



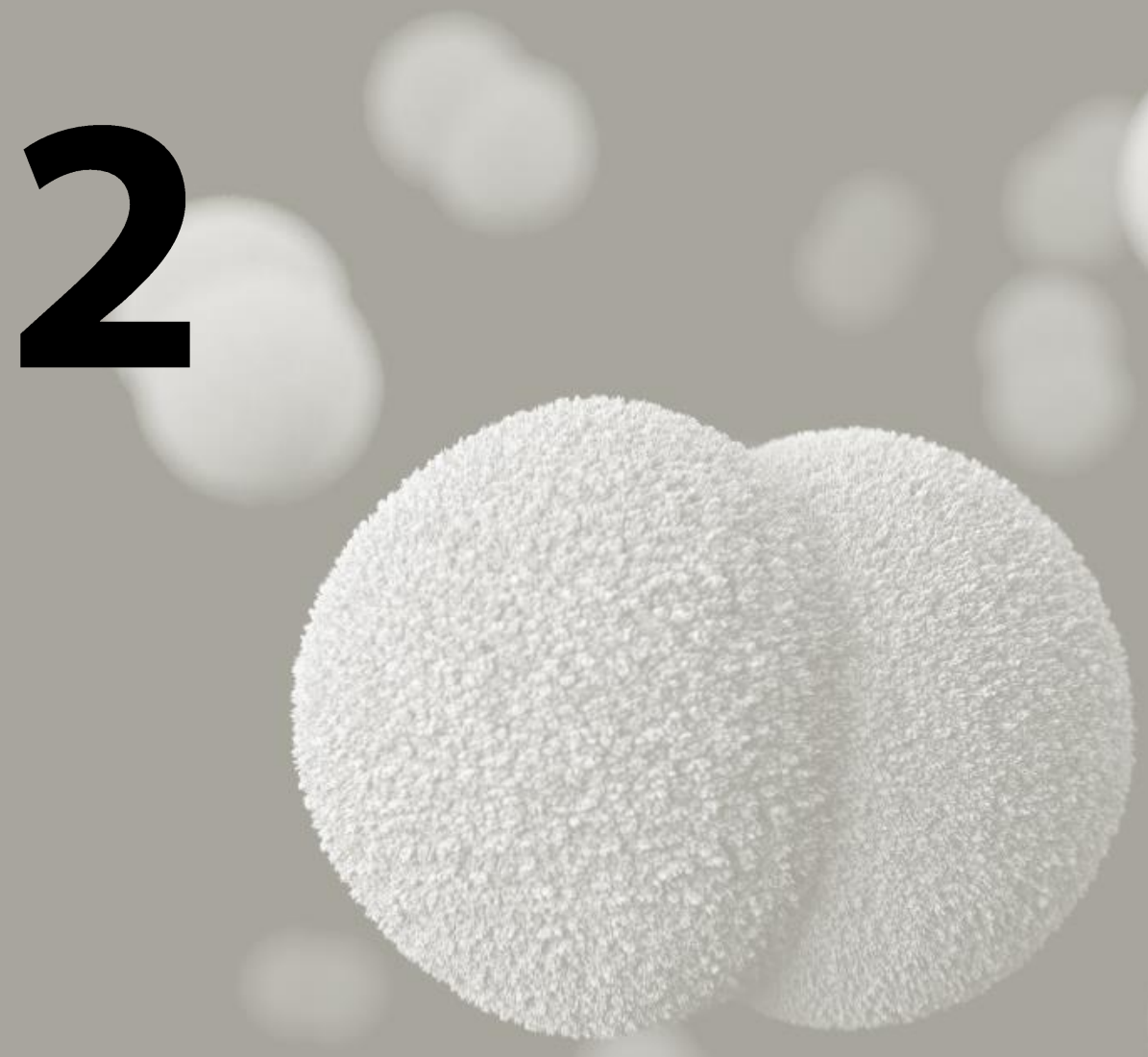




\section{Despite excellent test characteristics of the}

cobas $^{\circledR} 4800$ CT/NG assay, detection of oropharyngeal Chlamydia trachomatis and Neisseria gonorrhoeae remains challenging

Julius M. van Niekerk*, Brian M.J.W. van der Veer*, Christian J.P.A. Hoebe, Jeroen van de Bovenkamp, Christel van Herk, Inge H.M. van Loo, Lieke B. van Alphen, Petra F.G. Wolffs 


\section{Abstract}

\section{Objectives}

Oropharyngeal Chlamydia trachomatis (CT) and Neisseria gonorrhoeae (NG) infections are common but few commercial nucleic acid amplification tests (NAATs) specify extragenital samples as intended use. The test characteristics of the $\operatorname{cobas}^{\circledR} 4800 \mathrm{CT} / \mathrm{NG}$ assay are evaluated for oropharyngeal swabs.

\section{Methods}

The technical validation includes analysis of the specificity, sensitivity, dynamic range, linearity, efficiency and precision. The probability of detection curve combined with historical data enables estimation of potentially missed diagnoses. A clinical evaluation has been performed on a subset of 2798 clinical samples available from routine diagnostics. Results of the cobas ${ }^{\circledast} 4800$ were compared with in-house CT/NG PCR assays. Discrepant samples were tested with resolver assays and these results were considered decisive.

\section{Results:}

No cross-reactivity was seen in the analytical specificity analysis. High linearity $(\geq 0.983$ $R^{2}$ ), efficiency (89\%-99\%), and precision (0.1-0.9 $C_{t}$-value) were seen for both CT/NG. The limit of detection in oropharyngeal samples was $3.2 \times 10^{2} \mathrm{IFU} / \mathrm{mL}$ for $\mathrm{CT}$ and $6.7 \times 10^{2}$ $\mathrm{CFU} / \mathrm{mL}$ for NG. Estimates on potentially missed diagnoses were up to $7.2 \%$ for $\mathrm{CT}$ and up to $24.7 \%$ for NG. Clinical sensitivity and specificity were evaluated with 25 CT-, 86 NG positive and 264 negative samples, resulting in $100 \%$ and $99.6 \%$ for CT and $100 \%$ and $96.7 \%$ for NG respectively.

\section{Conclusion:}

The findings in this study demonstrate the utility of the cobas ${ }^{\circledR} 4800 \mathrm{CT} / \mathrm{NG}$ assay for oropharyngeal samples. Despite being a highly accurate test, the range of reported $\mathrm{C}_{\mathrm{t}}$ values especially for NG suggests relatively low oropharyngeal loads. Hence, consistent detection over the full range of oropharyngeal loads could be impaired. 


\section{Introduction}

Chlamydia trachomatis (CT) and Neisseria gonorrhoeae (NG) are the most common diagnosed bacterial sexual transmitted infections (STI) globally (1). When left untreated, these STI may have significant health implications (2), including chronic pelvic pain, ectopic pregnancy, tubal infertility, pelvic inflammatory disease, and Fitz-Hugh-Curtis syndrome in women, and epididymitis, or epididymo-orchitis in men $(3,4)$.

Previous studies demonstrated the importance of screening of extragenital sites for CT/NG infections, as up to $6.6 \%$ of CT and up to $36.4 \%$ of NG can be missed in men who have sex with men when oropharyngeal sampling is not performed (5), and up to $92.2 \%$ and $73.8 \%$ of oropharyngeal CT/NG have negative urethral tests (6).

Few of the currently commercially available nucleic acid amplification tests (NAATs) specify oropharyngeal samples for their intended use. The FDA has granted clearance of both the Xpert CT/NG to Cepheid, and Aptima Combo 2 Assay to Hologic for extragenital testing (7). Concerns about oropharyngeal testing are mainly related to the ability of assays to cross-react with non-gonoccocal Neisseria species, leading to false-positive results (8-11). Complicating matters even more is that oropharyngeal CT/NG loads have been demonstrated to be lower than urogenital and rectal loads (12, 13). Nevertheless, current laboratory standards recommend NAATs for the detection of oropharyngeal CT/NG if a laboratory has established specifications for performance characteristics in accordance to present regulations (8).

Recent efforts by Roche have shown promising results for the cobas ${ }^{\circledR} 6800$ for CT/NG screening (Roche Diagnostics, Indianapolis, IN, USA) of oropharyngeal and anorectal swab specimens, and are the first CE-IVD test with a claim for the detection of CT/NG from extragenital samples (14). However, the commonly used cobas $^{\circledR} 4800$ (Roche) has not been validated for the detection of oropharyngeal CT/NG, despite its high analytical $(9,15)$ and clinical specificity in urogenital (15-19) and rectal samples $(20)$ , and the $\operatorname{cobas}^{\circledR} \mathrm{CT} / \mathrm{NG}$ assay being a FDA-cleared NAAT (8). Although the more recently launched cobas $^{\circledR} 6800$ uses an optimized protocol, it is stated that the primer and probe design are identical for the cobas $^{\circledR} 4800$ (14). Therefore, the aim of this study is to assess the validity of the $\operatorname{cobas}^{\circledast} 4800$ for oropharyngeal swabs for the detection of CT/NG. The 
secondary goal of the study is to provide an estimate of missed low level positives, based on load-related probability of detection.

\section{Methods}

\section{Technical validation}

The cobas $^{\circledast} 4800$ is a qualitative NAAT for CT/NG detection on an automated system that implements DNA isolation, polymerase chain reaction (PCR) preparation, and $\mathrm{qPCR}$ of the cryptic plasmid and outer-membrane protein A for CT detection, including the detection of the Swedish new variant strain and the genomic target DR9-region (wildtype or highly conserved sequence variant) for NG (15). The technical validity is based on the analysis of the dynamic range (linearity and efficiency), the probability of detection (POD) curve, the limit of detection (LOD), precision (intra- and inter-assay variation) and analytical specificity.

The dynamic range was determined using three $C T$ serovars $(D, E, F)$ and three NG strains (NG-MAST ST2992, ST11461, ST14764) cultured as described previously (21, 22). Dilution series in $\operatorname{cobas}^{\oplus} \mathrm{PCR}$ Media were made ranging from $1.6-4.3 \times 10^{8}$ till 1.6$4.3 \times 10^{0} \mathrm{IFU} / \mathrm{mL}$ for $\mathrm{CT}$ and $10^{7}-10^{\circ} \mathrm{CFU} / \mathrm{mL}$ for NG in triplicate (23). Linear regression was performed to determine the dynamic range, defined as the range in which at least one replicate was positive, and efficiency in GraphPad Prism version 5.03 (GraphPad Software, San Diego, USA). The strain with the shortest dynamic range was spiked in triplicate in aliquots of a CT/NG negative oropharyngeal swab pool, resulting in concentration series of $10^{5}-10^{3}-10^{2}-10^{1}-10^{0} \mathrm{IFU} / \mathrm{mL}$ or $\mathrm{CFU} / \mathrm{mL}$. The series were tested at seven separate days resulting in 21 measurements per concentration to calculate the proportion of positive samples and a sigmoidal curve fit was used to construct a PODcurve. The LOD, defined as 95\% detection, was interpolated from the POD curve. Precision of the assay is described as the standard deviation (SD) as a measure of variation of which $\leq 1 C_{t}$-value is considered acceptable. This was determined on three separate days by adding two additional samples for low $\left(10^{2}\right.$ IFU/CFU $\left./ \mathrm{mL}\right)$, moderate $\left(10^{3}\right.$ IFU/CFU $/ \mathrm{mL})$, and high $\left(10^{5} \mathrm{IFU} / \mathrm{CFU} / \mathrm{mL}\right)$ concentrations. The analytical specificity of the cobas $^{\oplus} 4800$ was tested with a panel of 39 bacterial strains based on genetic similarity with $\mathrm{CT} / \mathrm{NG}$ or potential presence in the oropharynx $\left(\geq 10^{6} \mathrm{IFU} / \mathrm{mL}\right.$ or $\left.\mathrm{CFU} / \mathrm{mL}\right)$, not 
CT/NG, including C. muridarum (1), C. pneumoniae (1), E. coli (1), H. influenzae (2), $M$. catarrhalis (1), N. cinerea (1), N. denitrificans (1), N. elongata (1), N. flavescens (1), N. lactamica (2), N. meningitidis (3), N. mucosa (7), N. perflava (1), N. polysaccharea (1), N. subflava (14), S. aureus (1).

\section{Clinical evaluation}

The clinical evaluation is based on a retrospective sample selection from 24 August 2017 until 19 June 2018. In routine diagnostics, 2798 oropharyngeal swab samples (collected and stabilized with cobas $^{\oplus}$ PCR media) were screened for CT/NG in using the cobas $^{\circledast} 4800$ and stored at $-20^{\circ} \mathrm{C}$. Based on routine diagnostic results, 28 were CT positive and 92 NG positive resulting in a positivity rate of $1.0 \%$ and $3.3 \%$ respectively. All CT and NG positive samples were included and 264 consecutive CT/NG negative oropharyngeal samples from 28 August 2017 till 2 October 2017. The original results of the cobas $^{\circledR}$ were used for this study. Of nine samples, no residual sample was available and were therefore excluded, leaving 25 CT positive and 86 NG positive samples for analysis. Residual sample material of routine diagnostics was used in comparator tests targeting the cryptic plasmid for CT and porA pseudogene for NG $(24,25)$. QPCRs were performed as described previously (25). Discrepancy analysis was performed by resolver tests; the Versant kPCR CT/GC (Siemens Healthcare Diagnostics, Tarrytown, USA) in conjunction with an in-house opa qPCR for NG, by an independent technician ( $\mathrm{CvH}$ ) (26). All tests were performed within 2 years, which has previously been validated to hardly impact CT DNA detection (27). The comparator assay results were considered decisive, thereby classifying discrepant samples as true-positive or true-negative. After discrepancies were resolved sensitivity, specificity, positive predictive value (PPV), and negative predictive value (NPV) were determined.

\section{Estimation of undetected CT/NG}

Load values of interpolated cobas $^{\circledR} C_{t}$-values of $C T$ or NG positive oropharyngeal swabs $(n=796)$ between 2012 till 26 June 2019 were binned with intervals of $0.2 \log _{10}$ and observed frequencies were plotted in a histogram. These frequencies were divided by 
the mean POD per bin and subtracted by the observed frequency. This provides a rough estimate of missed low level positives, based on load-related probability of detection.

\section{Standard curves Chlamydia trachomatis}

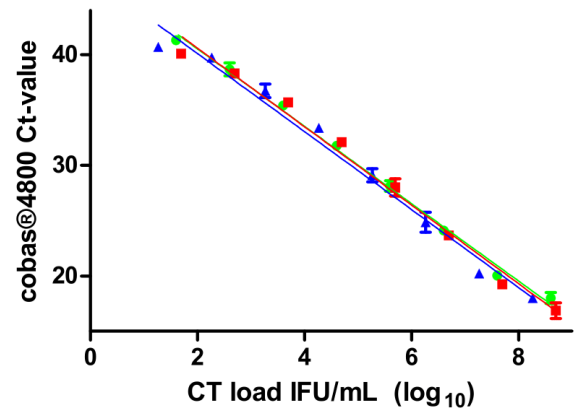

Standard curves Neisseria gonorrhoeae

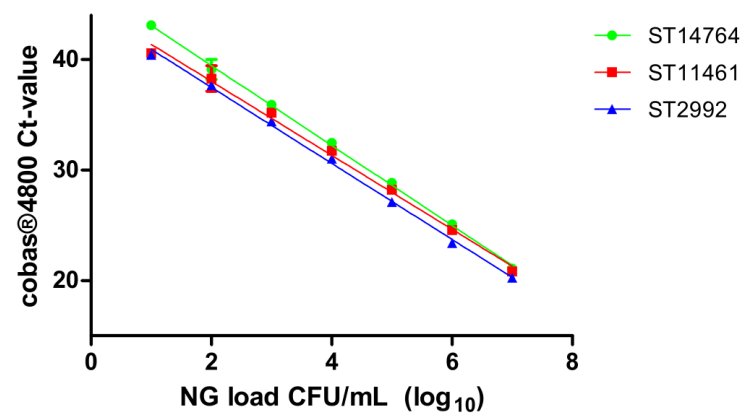

Figure 1 Standard curves of CT and NG. The dots represent the mean $\mathrm{C}_{\mathrm{t}}$-value and the error bars the standard deviation of the three independent replicates. In both CT and NG standard curves none of the $10^{\circ}$ were positive.

\section{Results}

\section{Technical validation}

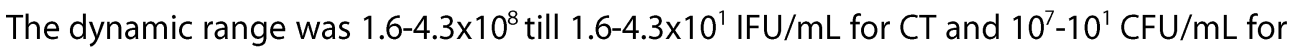
NG (Figure 1). In 8/18 CT samples with a load $>10^{7} \mathrm{IFU} / \mathrm{mL}$ false-positive NG results with high $C_{\mathrm{t}}$-values (range $C_{\mathrm{t}}$-value: 36.9-41.4) were observed. The assay demonstrated high linearity $\left(\geq 0.983 R^{2}\right)$ and efficiency (89\%-99\%) for both CT/NG. Serovar F and ST14764 were used for the POD curve as only $1 / 3$ replicates was positive at $10^{1} 1 \mathrm{FU} / \mathrm{CFU} / \mathrm{mL}$ in contrast with the other CT serovars and NG strains (2/3). All POD curve replicates $(n=21)$ of $10^{3}$ and $10^{5}$ (IFU or CFU per $\mathrm{mL}$ ) were detected by the assay (Figure 2). At $10^{2} \mathrm{IFU} / \mathrm{mL}$, $76.2 \%(16 / 21)$ of the CT replicates were positive, whereas at $10^{2} \mathrm{CFU} / \mathrm{mL}$ only $57.1 \%$ $(12 / 21)$ of the NG replicates were positive. Only $9.5 \%(2 / 21)$ for both CT and NG at $10^{1}$ and none at $10^{\circ}$ were positive. The interpolated LOD was $3.2 \times 10^{2} \mathrm{IFU} / \mathrm{mL}$ for CT and $6.7 \times 10^{2} \mathrm{CFU} / \mathrm{mL}$ for NG in oropharyngeal samples (Figure 2). Variation of qPCR results of oropharyngeal samples was tested with $10^{3}, 10^{5} \mathrm{IFU}$ or CFU/mL at three separate days; $10^{2}$ was excluded as it is below the LOD. The assay demonstrated high precision for both $\mathrm{CT}$ and $\mathrm{NG}$ as the demonstrated SD ranged from 0.2-0.7 $C_{t}$-value for all concentrations 
and similar results were found for the repeatability (SD 0.1-0.9 $C_{t}$-value). A $100 \%$ specificity was seen as none of the strains in the test panel showed positive amplification.

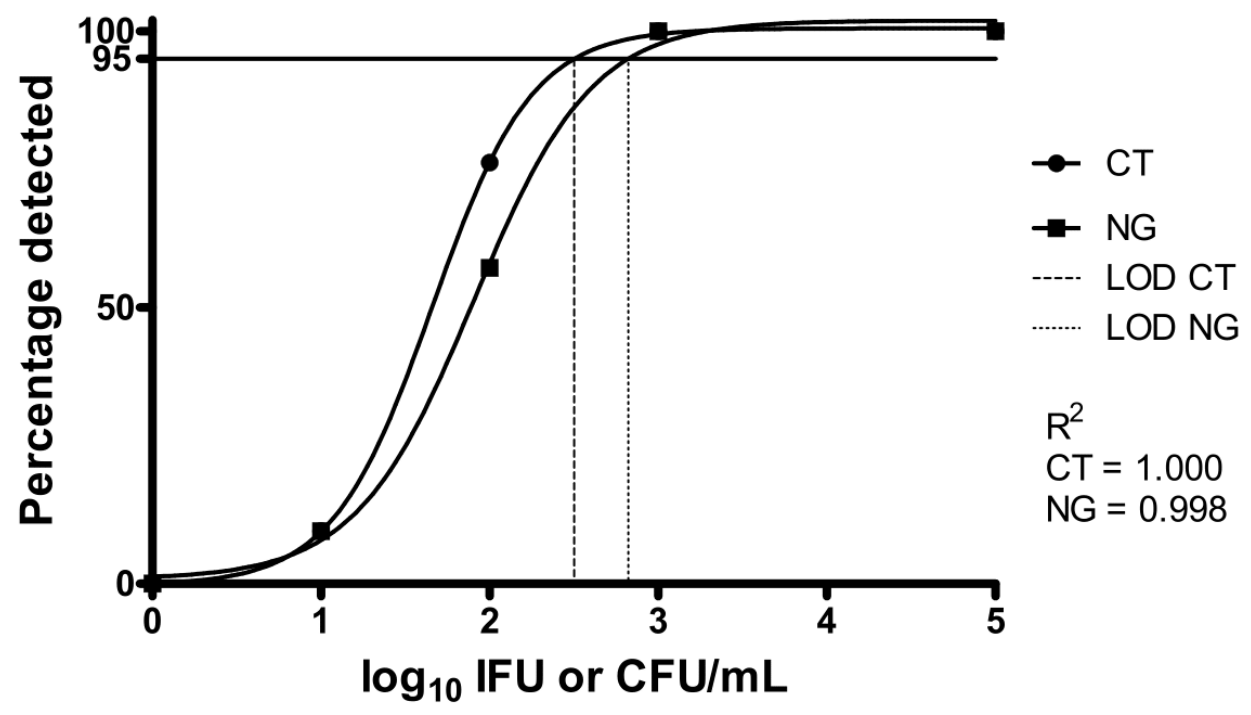

Figure 2 CT and NG POD curve of oropharyngeal samples. Dots (CT) and squares (NG) represent the proportion of positive replicates per concentration. The LOD was interpolated from the sigmoidal curve fit at $95 \%$ detection. For CT the LOD was $3.2 \times 10^{2} \mathrm{IFU} / \mathrm{mL}$ and $6.7 \times 10^{2} \mathrm{CFU} / \mathrm{mL}$ for NG.

\section{Clinical evaluation}

Sensitivity and specificity for CT detection were $100 \%$ and $99.6 \%$ and for NG $100 \%$ and 96.7\% respectively. Almost all CT results (288/289) were concordant except one $C_{\mathrm{t}}$-value 40.1 which was negative in the comparator assays and therefore considered false positive (Table 1). Most (326/350) of the NG results were concordant but 24 NG positive samples $\left(C_{t}\right.$-value range 31.4-39.6) were discordant in the in-house porA qPCR. Fifteen of the 24 discordant NG positive samples were confirmed with the resolver assay, whereas the remaining nine were not, and considered false positive. One sample had a $C_{t}$-value above the LOD and was Sanger sequenced, confirming the NG positive result of the cobas $^{\circledR} 4800$. 
Table 1 Clinical validity for CT and NG detection of the cobas ${ }^{\circledR} 4800$

\begin{tabular}{|c|c|c|c|c|}
\hline \multicolumn{5}{|c|}{ Cobas $^{\circledast} 4800$} \\
\hline Chlamydia plasmid & & Positive & Negative & \\
\hline \multirow[t]{3}{*}{ and Versant kPCR } & Positive & 24 & 0 & 24 \\
\hline & Negative & 1 & 264 & 265 \\
\hline & & 25 & 264 & 289 \\
\hline N. gonorrhoeae porA, & & Positive & Negative & \\
\hline \multirow[t]{3}{*}{ Versant kPCR and opa } & Positive & 77 & 0 & 77 \\
\hline & Negative & $9^{a}$ & 264 & 273 \\
\hline & & 86 & 264 & 350 \\
\hline
\end{tabular}

${ }^{a}$ Contains one Sanger sequence confirmed sample

\section{Estimated undetected CT/NG in oropharyngeal swabs}

Theoretical missed oropharyngeal infections were estimated using the POD curve and the observed CT/NG bacterial loads (Figure 3). For CT, up to $7.2 \%$ of oropharyngeal infections could go undetected and up to $24.7 \%$ for NG. 

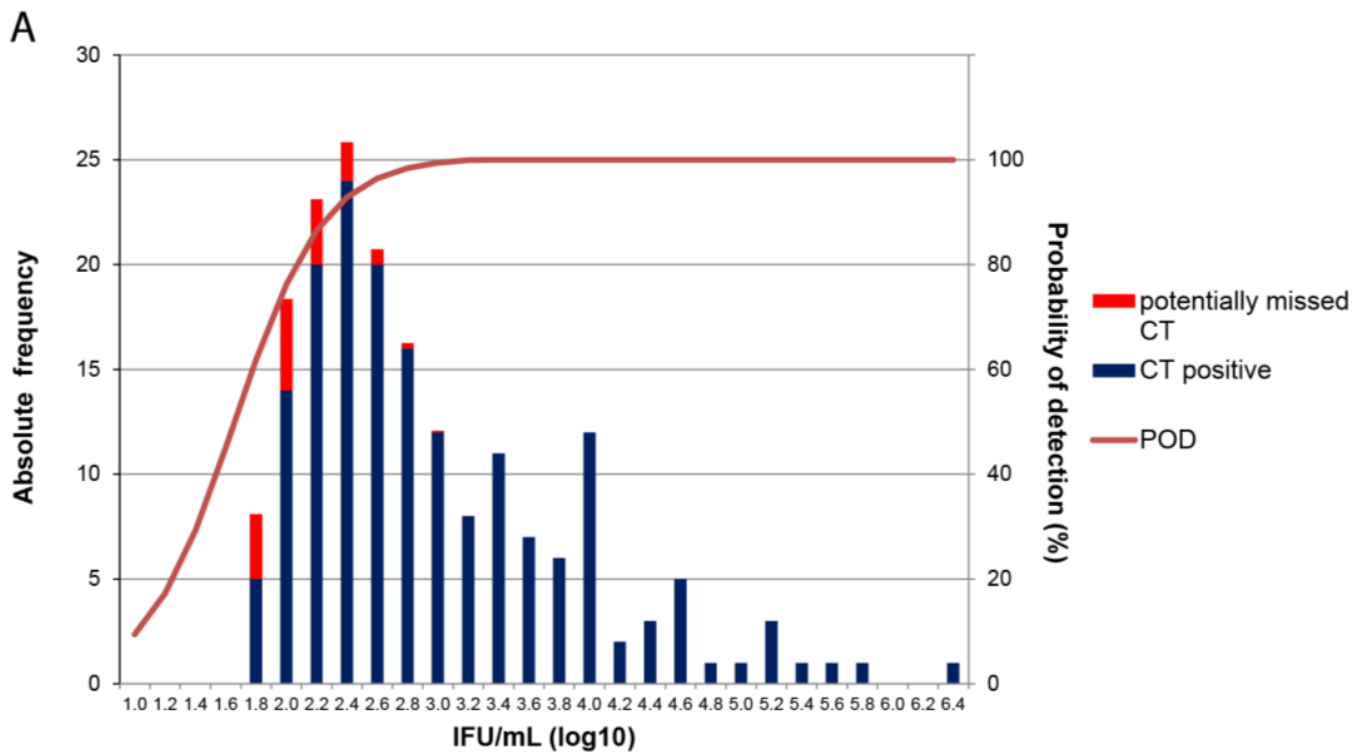

B

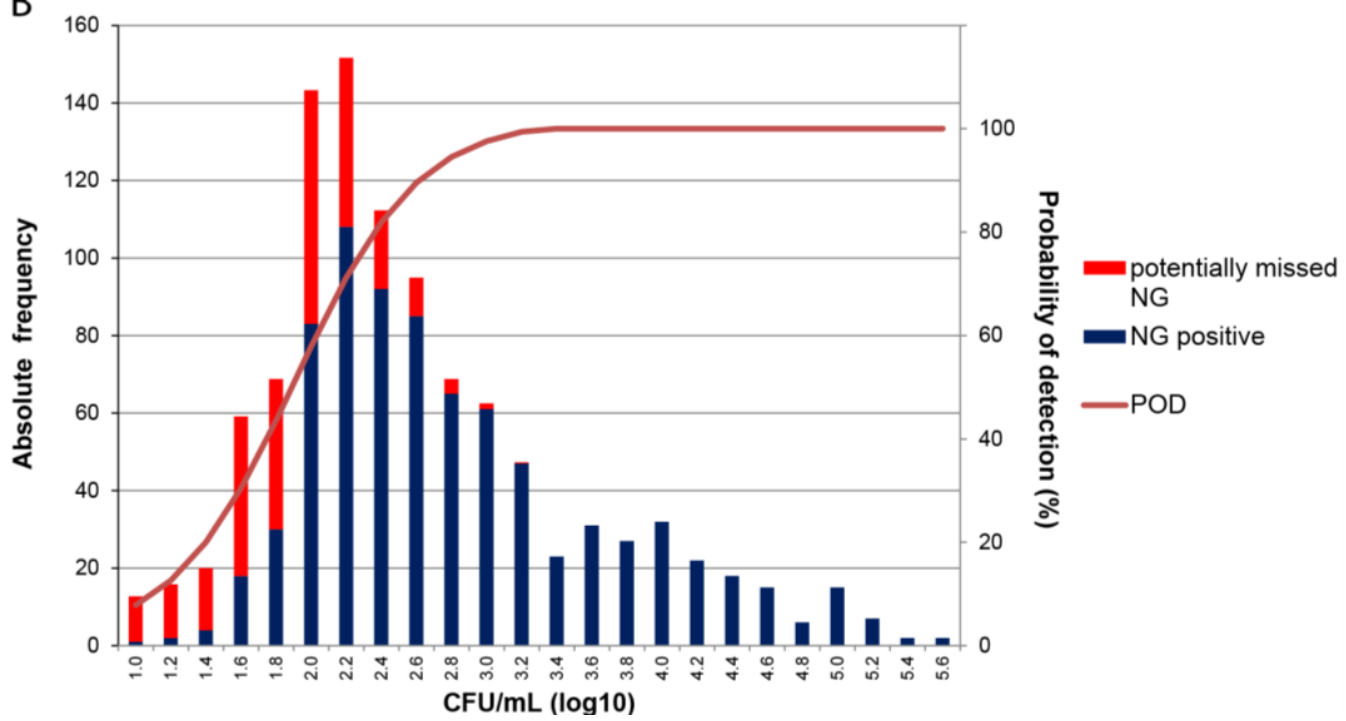

Figure 3 Potentially missed oropharyngeal CT/NG diagnoses. Absolute frequencies of oropharyngeal CT/NG diagnoses divided by probability of detection per bin. Loads are divided in bins of $0.2 \log 10$. In red: potentially missed diagnoses. Percentage potentially missed: amount of missed diagnoses / expected frequency. A) Potentially missed CT diagnoses $7.2 \%$ (13.5/(174+13.5)). B) Potentially missed NG diagnoses $24.7 \%(261 /(796+261))$. 


\section{Discussion}

For CT, the cobas $^{\circledR} 4800$ results demonstrate excellent technical performance characteristics, as evidenced by the high precision, the LOD, and the analytical specificity analysis. When assessing the technical performance with CT spiked samples, false positive NG results were observed in samples with $>10^{7} \mathrm{IFU} / \mathrm{mL}\left(C_{t}\right.$ 20.4). This phenomenon is likely caused by the high intensity fluorescent signal from the CT reporter dye overlapping into the NG detection channel. To estimate the impact of these potential false-positive results, we analysed all clinical routine diagnostic results in our laboratory between 2012-June 2019, but CT loads required to cause this phenomenon have not occurred. Therefore, spectral bleed-through is considered to be of limited clinical relevance. The clinical evaluation has also shown very good assay performance, with sensitivity for CT of $100 \%$, specificity of $99.6 \%$, PPV of $96 \%$, and NPV of $100 \%$. Although, the technical performance characteristics of the comparator assays are unknown for oropharyngeal samples. Lastly, the true specificity is likely underestimated as only a limited number of negative samples within the reported time period has been included, as further discussed under the limitations of the study.

Accordingly, the assay displays a very good performance for Neisseria gonorrhoeae. The analytical sensitivity demonstrates a LOD of $6.65 \times 10^{2} \mathrm{CFU} / \mathrm{mL}\left(\mathrm{C}_{\mathrm{t}} 36.5\right)$, with the lower limit of the dynamic range being $10^{1} \mathrm{CFU} / \mathrm{mL}$. Importantly, no crossreactivity was observed in the analytical specificity analysis. Likewise, a high precision has been observed. The clinical evaluation exhibited an excellent sensitivity (100\%) and NPV (100\%). Nine cobas $^{\circledR}$ positive samples could not be confirmed with the comparator assays. Eight of these samples had low loads $\left(C_{t}\right.$-values 38.7-39.3). Non-detection in this range of loads can be a matter of probability, as illustrated in Figure 2. The effects of DNA degradation could contribute in non-detection. However, the extent of degradation is likely limited, as specimens are collected and stabilized with cobas $^{\circledast}$ PCR media, and stored at $-20^{\circ} \mathrm{C}$. The remaining unconfirmed cobas ${ }^{\circledast}$ positive sample had a $\mathrm{C}_{\mathrm{t}}$ value of 36.4. Identification through Sanger sequencing demonstrated a NG nonetheless, disproving cross-reactivity. Hence, the definite specificity and PPV should be $97.1 \%$ and $90.7 \%$ respectively, instead of the reported $96.7 \%$ and $89.5 \%$ in Table 1 . 
However, the results on specificity and PPV should be interpreted in accordance with the limitations discussed below.

Previous studies on urine and endocervical swabs have shown equal performance characteristics of the cobas $^{\circledR}$ CT/NG assay in comparison to two FDA approved NAATs, with sensitivities and specificities ranging between $89.1-97.6 \%$ and 99.5-100\% for CT, and $95.6-100 \%$ and $99.7-100 \%$ for NG $(16,28)$. To best of our knowledge, only one study has performed a head-to-head comparison of the analytical sensitivity of the cobas $^{\circledR} 4800$ CT/NG assay to three FDA approved NAATs, for Chlamydia trachomatis (29). Self-collected vaginal swabs and first void urine were spiked with a CT dilutions series. This study showed a comparable sensitivity to the Abbott RealTime $\mathrm{CT} / \mathrm{NG}$ assay and BD Probetec CT/NG, and possibly a sensitivity disadvantage compared to the Gen-Probe Aptima Combo 2 (AC2). However, this disadvantage in sensitivity compared to the AC2 did not become statistically apparent in the aforementioned clinical evaluations $(16,28)$. Unfortunately, no comparisons of analytical sensitivity are available for NG. With the limited available data, there are no indications of

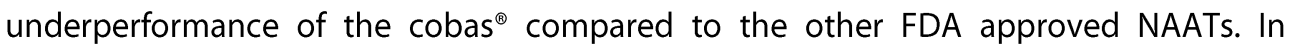
addition, NAATs are the recommended test for the detection of CT/NG infections, due to their overall sensitivity and specificity compared to other tests available, including culture (8).

Taking into account previous research indicating lower oropharyngeal CT/NG loads compared to urogenital and anorectal sites $(12,13)$, historical data on oropharyngeal CT/NG positive samples were reviewed. Of note, the NG load distribution curve peaks between $C_{t} 38-39$, with $469 / 797$ samples (58.8\%) having a $C_{t}$-value $\geq 36.5$ (load bin <2.8), and 301 samples $\geq 38.0$ (37.8\%, load bin <2.4). Given that the probability of detection declines rapidly with lower loads, the true number of positive samples in the range below the LOD are underrepresented. To estimate missed low level positives, a POD-curve was constructed (Fig. 2), which enables division of observed frequencies by their respective probability of detection, which provides a rough estimate of potentially missed diagnoses. These results suggest that up to $7.2 \%$ of oropharyngeal CT could go undetected, and possibly up to $25 \%$ of NG. As exemplified by the load distribution 
curves, it could be argued that the probability to consistently detect oropharyngeal CT/NG across the full range of loads might be a more common problem, likely across multiple platforms. Although the true extent of potentially missed diagnoses is subject to a large degree of measurement uncertainty discussed under the limitations, this study aims to point out that a considerable number of CT/NG could go undetected. If CT/NG loads were to be clinically relevant across the entire range of loads, clinical diagnostics and public health would benefit from more sensitive methods and/or controlling for pre-analytical factors that could improve sample collection.

Lastly, there is an ongoing debate on whether or not confirmatory testing is required for oropharyngeal NG results (18). The IUSTI guideline recommends confirmatory testing for extragenital samples, alongside a strict local evaluation of the performance on extragenital samples, before introduction of a NAAT. If the NAAT's PPV does not exceed $90 \%$, confirmatory testing is recommended (4). The CDC recommends that consideration should be given to retest samples with an alternate target assay, when NAATs are used which are able to cross-react with non-gonococcal Neisseria species (8). From the perspective of oropharyngeal NG loads as presented in this study, the authors would argue that load-related inability to confirm with a secondary test must be taken into consideration in this debate, and might be under-recognized in this discussion.

This study has several limitations. First, only a fraction of negative samples within the reported time period has been assessed in this study $(<10 \%$, i.e. $264 / 2773$ for CT, and 264/2712 for NG). This especially impacts the specificity, causing an underestimation. Hence, it is important to note that specificity data in this study cannot be extrapolated to different populations with known disease prevalence to calculate NPV or PPV. The PPV in this study is unaffected, as all cobas $^{\circledR}$ positive samples were included. The impact on sensitivity and NPV is probably small, as the prior probability of disease is small. Hence, the majority of negative samples will be 'true negatives'. Second, there are several factors that could cause significant measurement uncertainty in estimating the potentially missed diagnoses: (i) the adequacy of sample collection, (ii) the estimation of the CT/NG loads used in the dilution series required to establish the 
calibration curves, (iii) not estimating the calibration curves with multiple strains, (iv) the sigmoidal curve fit. Lastly, no LODs or POD-curves have been established for either of the comparator assays used in this study.

Taken together, the findings in this study demonstrate the utility of the cobas $^{\circledast} 4800 \mathrm{CT} / \mathrm{NG}$ assay for oropharyngeal samples. This study consists of a comprehensive technical and clinical evaluation of the CT/NG assay, taking into account probability of detection, and offers estimations of potentially missed diagnoses. Despite being a highly accurate test, the range of $C_{t}$-values especially for NG suggest relatively low oropharyngeal loads. Hence, consistent detection over the full range of oropharyngeal loads could be impaired, possibly for multiple platforms and more sensitive methods are called upon. 


\section{References}

1. Rowley J, Vander Hoorn S, Korenromp E, Low N, Unemo M, Abu-Raddad LJ, et al. Chlamydia, gonorrhoea, trichomoniasis and syphilis: global prevalence and incidence estimates, 2016. B World Health Organ. 2019;97(8):548-+.

2. World Health Organization. Emergence of multi-drug resistant Neisseria gonorrhoeae - Threat of global rise in untreatable sexually transmitted infections Fact sheet. 2011.

3. Lanjouw E, Ouburg S, de Vries HJ, Stary A, Radcliffe K, Unemo M. 2015 European guideline on the management of Chlamydia trachomatis infections. International journal of STD \& AIDS. 2016;27(5):333-48.

4. Bignell C, Unemo M, European STIGEB. 2012 European guideline on the diagnosis and treatment of gonorrhoea in adults. Int J STD AIDS. 2013;24(2):85-92.

5. Kent CK, Chaw JK, Wong W, Liska S, Gibson S, Hubbard G, et al. Prevalence of rectal, urethral, and pharyngeal chlamydia and gonorrhea detected in 2 clinical settings among men who have sex with men: San Francisco, California, 2003. Clinical infectious diseases : an official publication of the Infectious Diseases Society of America. 2005;41(1):67-74.

6. Patton ME, Kidd S, Llata E, Stenger M, Braxton J, Asbel L, et al. Extragenital gonorrhea and chlamydia testing and infection among men who have sex with men--STD Surveillance Network, United States, 2010-2012. Clinical infectious diseases : an official publication of the Infectious Diseases Society of America. 2014;58(11):1564-70.

7. Food and Drug Administration. FDA clears first diagnostic tests for extragenital testing for chlamydia and gonorrhea 2019.

8. Centers for Disease Control and Prevention. Recommendations for the laboratory-based detection of Chlamydia trachomatis and Neisseria gonorrhoeae--2014. MMWR Recommendations and reports : Morbidity and mortality weekly report Recommendations and reports. 2014;63(Rr-02):1-19.

9. Tabrizi SN, Unemo M, Limnios AE, Hogan TR, Hjelmevoll SO, Garland SM, et al. Evaluation of six commercial nucleic acid amplification tests for detection of Neisseria gonorrhoeae and other Neisseria species. J Clin Microbiol. 2011;49(10):3610-5.

10. Marangoni A, Foschi C, Nardini P, Compri M, Cevenini R. Evaluation of the Versant CT/GC DNA 1.0 assay (KPCR) for the detection of extra-genital Chlamydia trachomatis and Neisseria gonorrhoeae infections. PLoS One. 2015;10(3):e0120979.

11. Palmer HM, Mallinson H, Wood RL, Herring AJ. Evaluation of the specificities of five DNA amplification methods for the detection of Neisseria gonorrhoeae. J Clin Microbiol. 2003;41(2):835-7.

12. van der Veer BMJW, Hoebe CJPA, Dukers-Muijrers NHTM, van Alphen LB, Wolffs PFG. Men and women have a similar $<$ em $>$ Neisseria gonorrhoeae $</$ em $>$ bacterial load: a comparison of three anatomical sites. Journal of Clinical Microbiology. 2020:JCM.01171-20.

13. Wijers J, Dukers-Muijrers N, van Liere G, Dirks J, Wolffs PFG, Hoebe C. Men and women have an equal oropharyngeal and anorectal Chlamydia trachomatis bacterial load: a comparison of three anatomic sites. J Infect Dis. 2019.

14. Marlowe EM, Hardy D, Krevolin M, Gohl P, Bertram A, Arcenas R, et al. High-Throughput Testing of Urogenital and Extragenital Specimens for Detection of Chlamydia Trachomatis and Neisseria Gonorrhoeae with Cobas((R)) CT/NG. Eur J Microbiol Immunol (Bp). 2017;7(3):176-86.

15. Rockett R, Goire N, Limnios A, Turra M, Higgens G, Lambert SB, et al. Evaluation of the cobas 4800 CT/NG test for detecting Chlamydia trachomatis and Neisseria gonorrhoeae. Sex Transm Infect. 2010;86(6):470-3.

16. Taylor SN, Liesenfeld O, Lillis RA, Body BA, Nye M, Williams J, et al. Evaluation of the Roche cobas(R) CT/NG test for detection of Chlamydia trachomatis and Neisseria gonorrhoeae in male urine. Sex Transm Dis. 2012;39(7):543-9.

17. Parra-Sanchez M, Palomares JC, Bernal S, Gonzalez MT, Sivianes N, Perez L, et al. Evaluation of the cobas $4800 \mathrm{CT} / \mathrm{NG}$ Test for detecting Chlamydia trachomatis and Neisseria gonorrhoeae DNA in urogenital swabs and urine specimens. Diagn Microbiol Infect Dis. 2012;74(4):338-42.

18. Hopkins MJ, Smith G, Hart IJ, Alloba F. Screening tests for Chlamydia trachomatis or Neisseria gonorrhoeae using the cobas 4800 PCR system do not require a second test to confirm: an audit of patients issued with equivocal results at a sexual health clinic in the Northwest of England, U.K. Sexually transmitted infections. 2012;88(7):495-7. 
19. Kumamoto $\mathrm{Y}$, Matsumoto T, Fujisawa M, Arakawa S. Detection of Chlamydia trachomatis and Neisseria gonorrhoeae in urogenital and oral specimens using the cobas(R) 4800, APTIMA Combo 2(R) TMA, and ProbeTec ET SDA assays. European journal of microbiology \& immunology. 2012;2(2):121-7.

20. Geelen TH, Rossen JW, Beerens AM, Poort L, Morre SA, Ritmeester WS, et al. Performance of cobas(R) 4800 and m2000 real-time assays for detection of Chlamydia trachomatis and Neisseria gonorrhoeae in rectal and self-collected vaginal specimen. Diagn Microbiol Infect Dis. 2013;77(2):101-5.

21. Janssen KJ, Hoebe CJ, Dukers-Muijrers NH, Eppings L, Lucchesi M, Wolffs PF. Viability-PCR Shows That NAAT Detects a High Proportion of DNA from Non-Viable Chlamydia trachomatis. PLoS One. 2016;11(11):e0165920.

22. van der Veer BM, Wolffs PF, Hoebe CJ, Dukers-Muijrers NH, van Alphen LB. Culture-free genotyping of Neisseria gonorrhoeae revealed distinct strains at different anatomical sites in a quarter of patients, the Netherlands, 2012 to 2016. Euro Surveill. 2018;23(50).

23. Bustin SA, Benes V, Garson JA, Hellemans J, Huggett J, Kubista M, et al. The MIQE guidelines: minimum information for publication of quantitative real-time PCR experiments. Clin Chem. 2009;55(4):611-22.

24. Hopkins MJ, Ashton LJ, Alloba F, Alawattegama A, Hart IJ. Validation of a laboratory-developed realtime PCR protocol for detection of Chlamydia trachomatis and Neisseria gonorrhoeae in urine. Sex Transm Infect. 2010;86(3):207-11.

25. Janssen KJH, Wolffs P, Lucchesi M, Dukers-Muijrers N, Hoebe C. Assessment of rectal Chlamydia trachomatis viable load in women by viability-PCR. Sex Transm Infect. 2020;96(2):85-8.

26. Kerndt PR, Ferrero DV, Aynalem G, Monga D, Wang S, Zhang N, et al. First report of performance of the Versant CT/GC DNA 1.0 assay (kPCR) for detection of Chlamydia trachomatis and Neisseria gonorrhoeae. J Clin Microbiol. 2011;49(4):1347-53.

27. van Dommelen L, Wolffs PF, van Tiel FH, Dukers N, Herngreen SB, Bruggeman CA, et al. Influence of temperature, medium, and storage duration on Chlamydia trachomatis DNA detection by PCR. Journal of clinical microbiology. 2013;51(3):990-2.

28. Van Der Pol B, Liesenfeld O, Williams JA, Taylor SN, Lillis RA, Body BA, et al. Performance of the cobas CT/NG test compared to the Aptima AC2 and Viper CTQ/GCQ assays for detection of Chlamydia trachomatis and Neisseria gonorrhoeae. J Clin Microbiol. 2012;50(7):2244-9.

29. Chernesky M, Jang D, Gilchrist J, Hatchette T, Poirier A, Flandin JF, et al. Head-to-head comparison of second-generation nucleic acid amplification tests for detection of Chlamydia trachomatis and Neisseria gonorrhoeae on urine samples from female subjects and self-collected vaginal swabs. J Clin Microbiol. 2014;52(7):2305-10. 


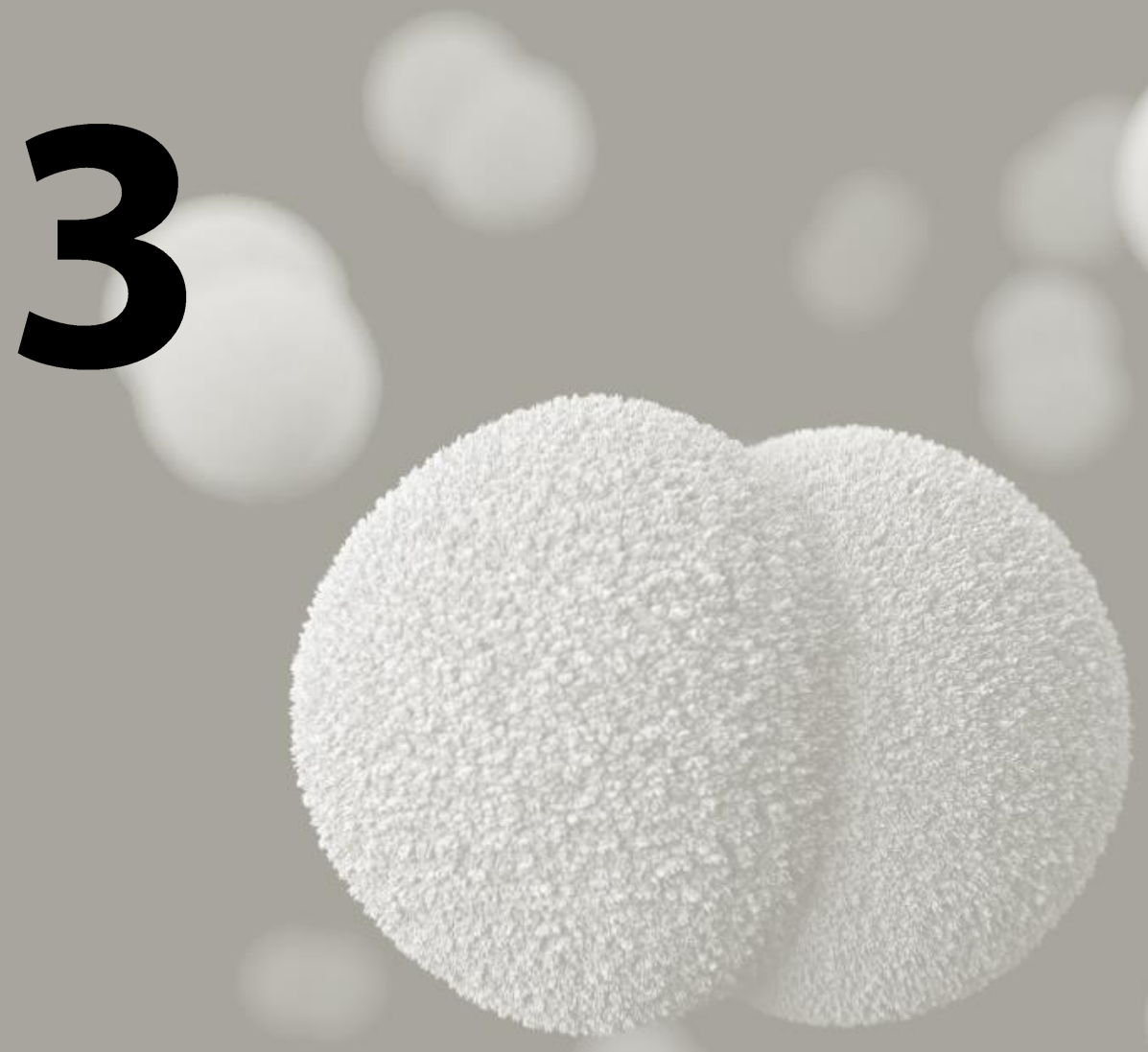




\section{Men and Women Have Similar Neisseria gonorrhoeae Bacterial Loads: a Comparison of Three Anatomical sites}

Brian M.J.W. van der Veer, Christian J.P.A. Hoebe, Nicole H.T.M. Duker-Muijrers,

Lieke B. van Alphen, Petra F.G. Wolffs 


\section{Abstract}

Neisseria gonorrhoeae is a common bacterial sexually transmitted infection (STI). Currently, there are limited data on the bacterial load in both men and women and on both genital and extragenital sites. Therefore, we quantified N. gonorrhoeae bacterial loads in a large population of women, heterosexual men, and men who have sex with men (MSM) at three different anatomical sites. N. gonorrhoeae-positive samples ( $n=1265)$ of STI clinic consultations ( $n=944$ ) were tested for N. gonorrhoeae with the Roche Cobas 4800 system, and quantification cycle (Cq) values were used as an inversely proportional measure for $N$. gonorrhoeae bacterial load after interpolation from a standard curve. Bacterial loads were compared between sample materials and sexes using $t$ tests. The following mean $N$. gonorrhoeae loads were observed: urine, $4.5 \pm 1.0 \log _{10} \mathrm{CFU} / \mathrm{ml}$; vaginal swabs, $4.3 \pm 1.1 \log _{10} \mathrm{CFU} / \mathrm{ml}$; anorectal swabs (women), $4.0 \pm 1.2 \log _{10} \mathrm{CFU} / \mathrm{ml}$; anorectal swabs (men), $4.5 \pm 1.3 \log _{10} \mathrm{CFU} / \mathrm{ml}$; oropharyngeal swabs (women), $2.8 \pm 0.9 \log _{10} \mathrm{CFU} / \mathrm{ml}$; and oropharyngeal swabs (men), $3.2 \pm 1.0 \log _{10} \mathrm{CFU} / \mathrm{ml}$. Oropharyngeal swabs had a significantly lower $N$. gonorrhoeae load $(P<0.001)$ than genital and anorectal samples. Loads did not differ between men and women. This is the first study that determined $N$. gonorrhoeae load in both women and men at three anatomical sites. The substantial $N$. gonorrhoeae load at all sample sites suggest that all sites may have transmission potential. However, the oropharyngeal site presents the lowest bacterial load. Men and women have a similar N. gonorrhoeae loads on separate anatomical sites, arguing for similar transmission potential and similar clinical relevance. 
Neisseria gonorrhoeae accounts for 87 million of the estimated 376 million new curable sexually transmitted infection (STI) cases worldwide every year (1). Limiting the transmission of $N$. gonorrhoeae is a major public health challenge. Many infections remain hidden to care, especially in women, because of stigma and their asymptomatic nature (2-5). Untreated N. gonorrhoeae infections can affect the vagina, cervix, urethra, the rectum, or the throat and could lead to severe sequelae. In women, N. gonorrhoeae can cause pelvic inflammatory disease, infertility, ectopic pregnancy, and maternal death (2). In men, urethral infections are frequently symptomatic and can cause urethritis with pus-like discharge, dysuria, and pain or swelling in one testicle (6). Current guidelines recommend urogenital testing for $N$. gonorrhoeae in both men and women (7-9). The need for extragenital testing is recognized for men who have sex with men (MSM) but not for women and heterosexual men. Aside from MSM, extragenital testing is only recommended for patients with an indication, such as a history of direct exposure by anal or oral sex or symptoms. The prevalence of extragenital N. gonorrhoeae is $3 \%$ to $21 \%$ in MSM but substantially lower in heterosexual men and women (0\% to $3 \%$ ) (10, 11). However, as current health care is mainly focused on genital testing, many $N$. gonorrhoeae infections remain hidden to care. Thus, $29 \%$ to $30 \%$ of N. gonorrhoeae infections in women and $14 \%$ of $N$. gonorrhoeae infections in heterosexual men will be missed if extragenital testing is not performed $(11,12)$. Hidden $N$. gonorrhoeae infections may facilitate ongoing transmission, although the extent of underdiagnosis and undertreatment of extragenital $N$. gonorrhoeae infections remains unknown (6, 10-13). In addition, oropharyngeal infections are thought to have a key role in antimicrobial resistance (AMR) development in $N$. gonorrhoeae because of the common presence of commensal Neisseria species that readily exchange DNA (14). Aside from poor access to health care and high-risk behavior, the transmissibility of $N$. gonorrhoeae can also be linked to bacterial load, as a higher inoculum dose in an experimental infection study resulted in higher infection probability $(2,15)$. The site of infection and symptoms could also be associated with $N$. gonorrhoeae bacterial load. Previous studies have demonstrated a higher $N$. gonorrhoeae load in urethral infections of symptomatic men and anorectal infections of symptomatic $\operatorname{MSM}(16,17)$. Lower N. gonorrhoeae load was 
observed in oropharyngeal swabs of MSM than in urethral and anorectal swabs $(16,17)$. However, these studies were relatively small, and no study has assessed the $N$. gonorrhoeae bacterial load in women in relation to symptoms. Therefore, the aim of this study was to assess the bacterial load of $N$. gonorrhoeae at three different anatomical sites (urogenital, anorectal, and oropharyngeal) in a large population of women, heterosexual men, and MSM visiting a large STI clinic. Bacterial loads were compared between sample materials and associations with symptoms were assessed. Results might provide insights in the transmission potential of different anatomical sites in women and men and might also inform testing guidelines for clinical practice.

\section{Material and methods}

\section{Study population and procedures.}

The South Limburg public health service STI clinic offers STI testing for patients with high-risk of acquiring an STI free of charge. Between January 2012 and July 2017, N. gonorrhoeae was diagnosed in 923 individuals, yielding data from 1,095 consultations for retrospective analysis. The $N$. gonorrhoeae positivity rates in this time frame were $1.7 \%$ in women, $1.7 \%$ in heterosexual men, and $12.0 \%$ in MSM. Patients were tested urogenitally for $N$. gonorrhoeae using first-void urine (men) or a self-collected vaginal swab (women). According to regular STI clinic guidelines, MSM were routinely and systematically tested at both anorectal and oropharyngeal sites (18). However, heterosexual men and women were tested extragenitally when reporting symptoms or receptive anal sex (women) or oral sex in the last 6 months (18). Patients were requested to collect a self-taken anorectal swab or a nurse-taken oropharyngeal swab for extragenital testing. A standardized medical and sexual history was routinely taken by nurses for all patients, including self-reported symptoms and sexual behavior in the last 6 months. Urogenital symptoms were defined as one or more of the following: genital discharge, bleeding, itching, swelling, pain, burning sensation, and more frequent urination. Anal symptoms were defined as rectal discharge, bleeding, pain, and itching. Any oropharyngeal symptoms were defined as symptomatic. We excluded samples $(n=$ 215) from consultations that had missing data on the Cobas 4800 CT/NG test (Roche Diagnostics, Basel, Switzerland) quantification cycle $(C q)$ value, sexual risk group, 
symptoms, and number of sex partners in the previous 6 months, leaving 944 consultations (800 individuals) and 1,265 samples for analysis (see Fig. S1 in the supplemental material).

\section{N. gonorrhoeae diagnosis and bacterial load.}

All samples were tested for N. gonorrhoeae with the Cobas 4800 CT/NG test. This assay targets a direct repeat region called DR-9 that is specific for $N$. gonorrhoeae (19). The Cobas test utilizes amplification using two sets of primers and probes to detect two highly conserved variations within the DR-9 region. Little variation is expected between N. gonorrhoeae strains, because this assay is not prone to copy number variation and the targets are highly conserved. Therefore, bacterial load can be quantified by interpolation of $\mathrm{C} q$ values to a standard curve with known concentrations of $N$. gonorrhoeae. For quantification, a standard curve was made for each specimen type (e.g., swab or urine) as the Cobas $4800 \mathrm{CT} / \mathrm{NG}$ test requires different volumes of input material (20). $N$. gonorrhoeae suspension from log-phase culture was spiked (100 $\mu \mathrm{l})$ and serially diluted 10-fold. A part of the suspension was plated to determine the amount of $N$. gonorrhoeae by counting the colonies resulting in $n$ CFU per $\mathrm{ml}$. To resemble urine samples, $5 \mathrm{ml}$ of an N. gonorrhoeae-negative male urine pool was added and stabilized before $N$. gonorrhoeae was spiked.

\section{Data analysis.}

We performed a retrospective data analysis of the bacterial loads of 1,265 N. gonorrhoeae-positive samples from 944 routine diagnostic consultations stratified by sample material and sexual risk group (data available in Data Set S1). We binned the number of sex partners in the previous 6 months in tertiles ( 0 to 2,3 to 7 , and $\geq 8$ ). Descriptive statistics were used to describe the proportion of concurrent N.gonorrhoeae infections at separate anatomical sites, symptoms, and number of sex partners per sexual risk group and sample material. Bacterial load values were $\log _{10}$ transformed for analyses and compared between sample materials using Student's $t$ tests (all combinations of two sample materials). Using multivariable linear regression, the 
bacterial load comparison between sample materials was adjusted for age, sexual risk group, symptoms, and number of sex partners. Bacterial loads were also compared per sample material between sexes with a Student's $t$ test. Using multivariable linear regression, the bacterial load comparison between sample materials and sexes was adjusted for age, sexual risk group, symptoms, and number of sex partners. Next, associations between symptoms (i.e., the dependent variable with the asymptomatic group as the reference group) and bacterial load (i.e., the outcome) were assessed. Therefore, univariable and multivariable linear regression analyses were performed on data stratified by sample material and sexual risk group. These models were adjusted for age and number of sex partners in multivariable linear regression. A $P$ value of less than 0.05 was considered statistically significant. All analyses were performed using $R$ statistical software, version 3.4.1 (R Foundation for Statistical Computing, Vienna, Austria). Figures were made using the R package ggplot2 version 3.1.0.

\section{Ethics.}

The Medical Ethical Committee of Maastricht University Medical Centre (MUMC+; number METC 2017-2-0251) approved the study protocol, as only deidentified retrospective data from standard care were used. Consultations were part of routine STI clinic procedures where individuals did not object to the use of their samples and data for research. For this study, we used two retrospective databases from the STI clinic and laboratory, with data collected within standard care. 


\section{Results}

\section{Proportion of extragenital positivity.}

N. gonorrhoeae was detected in 944 routine diagnostic consultations with 1,265 N. gonorrhoeae-positive samples, among which, 603 belonged to MSM, 144 to heterosexual men, and 197 to women (Table 1). In most patients, only one sample was N. gonorrhoeae positive, irrespective of sexual risk group $(73.6 \%$ of women, $98.6 \%$ of heterosexual men, and $64.8 \%$ of MSM) (Table 1). Some patients were N. gonorrhoeae positive at all three tested anatomical sites (55/944 [5.8\%]). Extragenital-only, anorectal, or oropharyngeal $N$. gonorrhoeae-positive samples were observed in 31.0\% (61/197) of women, $22.9 \%$ (33/144) of heterosexual men, and 73.8\% (445/603) of MSM who were diagnosed (Table 1). Of these, most of the women (49/61 [80.3\%]) and all heterosexual men were $N$. gonorrhoeae-positive only at the oropharyngeal site. 
Table 1 Characteristics of the study population by sexual risk group.

\begin{tabular}{lllll}
\hline Characteristic & $\begin{array}{l}\text { Women } \\
(\mathbf{n}=\mathbf{1 9 7})\end{array}$ & $\begin{array}{l}\text { Heterosexual men } \\
(\mathbf{n}=\mathbf{1 4 4})\end{array}$ & $\begin{array}{l}\mathbf{M S M}^{\mathbf{a}} \\
(\mathbf{n}=\mathbf{6 0 3})\end{array}$ & $\begin{array}{l}\text { Total } \\
(\mathbf{n}=\mathbf{9 4 4})\end{array}$ \\
\hline $\begin{array}{l}\text { Age (yr) (median [interquartile } \\
\text { range]) }\end{array}$ & $31(22-44)$ & $30(23-47)$ & $32(24-45)$ & $31(23-45)$ \\
& & & & \\
No of N. gonorrhoeae-positive & & & & \\
samples within one patient (\% & & & $64.8(391)$ & $71.8(678)$ \\
$[n])^{\mathrm{b}}$ & & & $28.0(169)$ & $22.4(211)$ \\
1 & $73.6(145)$ & $98.6(142)$ & $7.1(43)$ & $5.8(55)$ \\
2 & $20.3(40)$ & $1.4(2)$ & & \\
3 & $6.1(12)$ & $0.0(0)$ & $6.8(41)$ & $25.0(236)$ \\
& & & $32.8(198)$ & $22.0(208)$ \\
N.gonorrhoeae diagnosis (\% [n]) & & & $25.2(152)$ & $24.8(234)$ \\
Genital only & $43.7(86)$ & $75.7(109)$ & $10.8(65)$ & $9.9(93)$ \\
Anorectal only & $5.1(10)$ & $0.0(0)$ & $1.5(9)$ & $2.2(21)$ \\
Oropharyngeal only & $24.9(49)$ & $22.9(33)$ & $15.8(95)$ & $10.3(97)$ \\
Genital - anorectal & $14.2(28)$ & $0.0(0)$ & $7.1(43)$ & $5.8(55)$ \\
Genital - oropharyngeal & $5.1(10)$ & $1.4(2)$ & &
\end{tabular}

Symptoms (any site) (\% [n])

Asymptomatic

$40.1(79) \quad 38.9(56)$

$46.9(283) \quad 44.3(418)$

Symptomatic

$59.9(118) \quad 61.1(88)$

$53.1(320) \quad 55.7(526)$

Number of sex partners (\% [n])

$0-2$

$31.5(62) \quad 34.7(50)$

$18.2(110) \quad 23.5(222)$

3-7

$34.0(67) \quad 47.9(69)$

$43.6(263) \quad 42.3(399)$

$\geq 8$

$34.5(68)$

$17.4(25)$

$38.1(230) \quad 34.2(323)$

${ }^{a}$ MSM, men who have sex with men

${ }^{b} n$, number of consultations 


\section{N. gonorrhoeae bacterial loads compared between sample materials and sexes.}

In total, 269 urine samples (genital site, men), 136 vaginal swabs (genital site, women), 453 anorectal swabs, and 407 oropharyngeal swabs were available for analysis (Table 2). The mean bacterial loads per sample material were as follows: urine samples, $4.5 \pm 1.0$ $\log _{10}$ CFU/ml; vaginal swabs, $4.3 \pm 1.1 \log _{10}$ CFU/ml; anorectal swabs, $4.4 \pm 1.3 \log _{10}$ CFU/ml; and oropharyngeal swabs, $3.1 \pm 1.0 \log _{10}$ CFU/ml. Oropharyngeal swabs had a lower mean load (16- to 25 -fold lower) than all other sample materials ( $P<0.001, t$ tests), while no statistically significant difference was observed between the other sample materials. The observation of lower load in oropharyngeal swabs persisted even when corrected for age, sexual risk group, symptoms, and number of sex partners in a linear regression model $(P<0.001)$.

Table 2 Characteristics of the study population by sample material.

\section{$\%$ (no.) of samples}

Category

\begin{tabular}{lllll}
\hline Urine & Vaginal & Anorectal & Oropharyngeal & Total \\
$(n=269)$ & $(n=136)$ & $(n=453)$ & $(n=407)$ & $(n=1265)$
\end{tabular}

Sexual risk group

Women

$N A^{a}$

100 (136)

$11.5(52)$

$17.9(73)$

$20.6(261)$

Heterosexual men

$41.3(111)$

NA

$0.0(0)$

$8.6(35)$

$11.5(146)$

$\mathrm{MSM}^{\mathrm{b}}$

58.7 (158)

NA

88.5 (401)

73.5 (299)

$67.8(858)$

Symptoms

$\begin{array}{llllll}\text { Asymptomatic } & 23.0(62) & 31.6(43) & 75.1(340) & 88.9(362) & 63.8(807) \\ \text { Symptomatic } & 77.0(207) & 68.4(93) & 24.9(113) & 11.1(45) & 36.2(458)\end{array}$

\footnotetext{
${ }^{a}$ NA, not applicable, as no samples were collected as part of routine diagnostic procedures.

${ }^{\mathrm{b}} \mathrm{MSM}$, men who have sex with men.
} 
Stratified by sex, the mean bacterial loads were as follows: urine samples, $4.5 \pm$ $1.0 \log _{10}$ CFU/ml; vaginal swabs, $4.3 \pm 1.1 \log _{10}$ CFU/ml; anorectal swabs of women, $4.0 \pm$ $1.2 \log _{10}$ CFU/ml; anorectal swabs of men, $4.5 \pm 1.3 \log _{10}$ CFU/ml; oropharyngeal swabs of women, $2.8 \pm 0.9 \log _{10} \mathrm{CFU} / \mathrm{ml}$; and oropharyngeal swabs of men, $3.2 \pm 1.0 \log _{10}$ CFU/ml (Fig. 1). Anorectal and oropharyngeal swabs from women had a significantly lower load than those from men, 2.8-fold $(P=0.02)$ and 2.3-fold $(P=0.002)$, respectively. However, these differences were not statistically different when corrected for age, symptoms, and number of sex partners $(P=0.09$ and $P=0.06$, respectively).

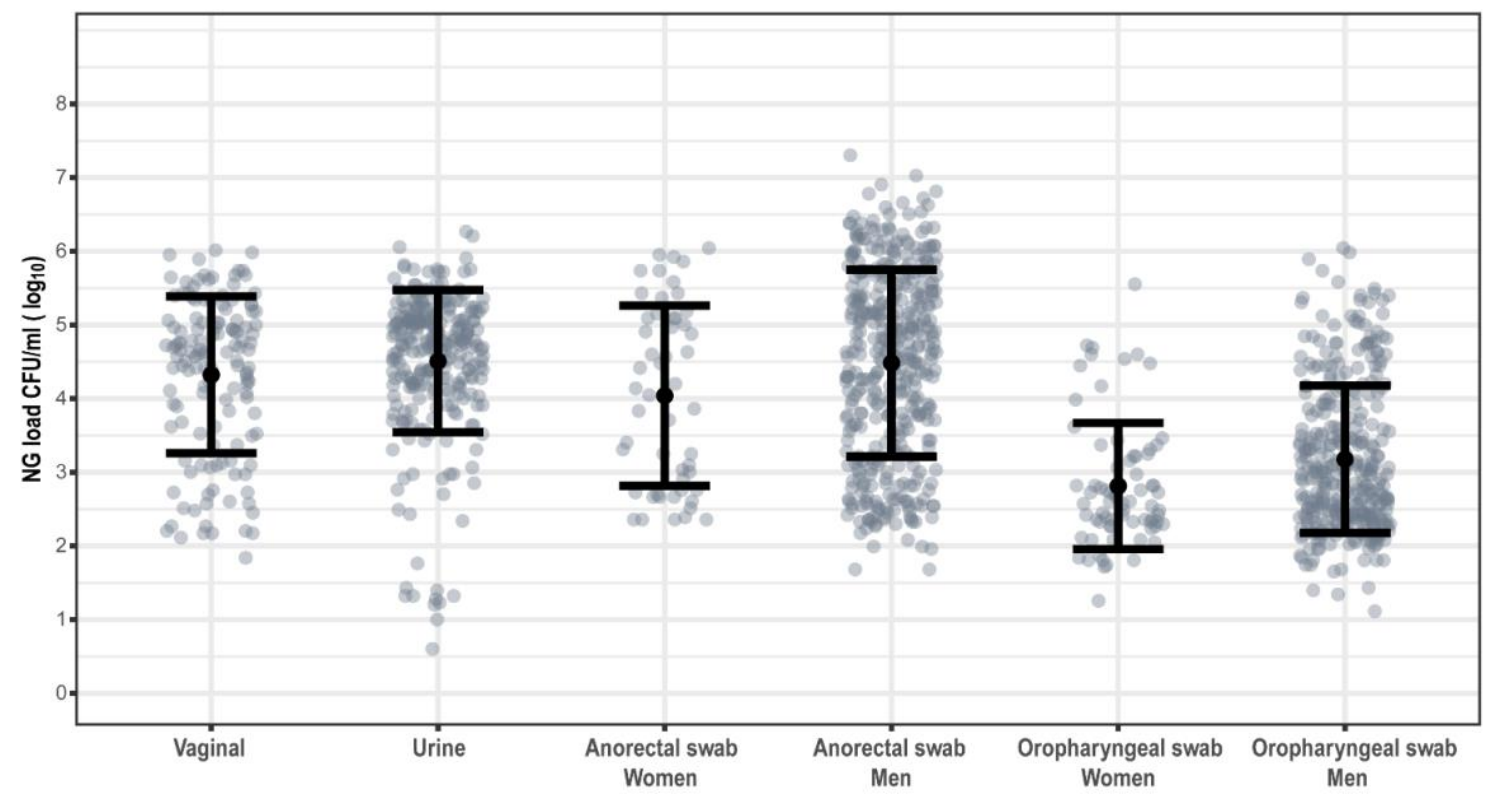

FIG 1 Neisseria gonorrhoeae bacterial loads stratified by sample material and sex. Oropharyngeal NG bacterial load was lower than genital and anorectal N.gonorrhoeae bacterial loads $(P<0.001)$. No significant difference was observed between the other sample materials. The small differences between women and men can be explained by differences in age, symptoms, and number of sex partners.

\section{Association between symptoms and $\mathbf{N}$. gonorrhoeae bacterial load.}

Most anorectal (75\%) and oropharyngeal (89\%) samples were from asymptomatic infections, but only a minority of the genital samples (23\% to $32 \%$ ) were from asymptomatic infections (Table 2). Genital symptoms were associated with a 3- to 5-fold higher bacterial load in urine samples from men (heterosexual men, $P<0.001 ; \mathrm{MSM}, P=$ 
0.03) but not in vaginal swabs from women $(P=0.26)$, in both univariable and multivariable linear regression models (Tables 3 to 5). Associations of oropharyngeal symptoms with $N$. gonorrhoeae bacterial load could not be assessed in heterosexual men, because no symptoms were reported (Table 4). In both women $(P=0.007)$ and MSM $(P<0.001)$, anorectal symptoms were associated with a higher $N$. gonorrhoeae bacterial load, 4- and 47-fold, respectively. In contrast, oropharyngeal symptoms were not associated with a higher $N$. gonorrhoeae load in either women $(P=0.99)$ or MSM $(P=$ 0.06). The results remained similar in analyses that were restricted to women (97/197) or MSM (595/603) who were tested at both genital and extragenital sites, including in an analysis corrected for the $N$. gonorrhoeae load at the concurrent sites (see Tables S1 and S2 in the supplemental material).

Table 3 Mean N. gonorrhoeae bacterial load and association of symptoms with N. gonorrhoeae bacterial load in women per sample material

\begin{tabular}{|c|c|c|c|c|c|}
\hline \multirow[b]{2}{*}{ Category } & \multirow[b]{2}{*}{$n$} & \multicolumn{2}{|l|}{$\mathrm{CFU} / \mathrm{ml}$} & \multirow[b]{2}{*}{ Adjusted $\Delta \log _{10}(95 \% \mathrm{Cl})$} & \multirow[b]{2}{*}{$\begin{array}{c}\text { Factor } \\
\text { load }\end{array}$} \\
\hline & & $\begin{array}{l}\text { Mean } \log _{10} \\
\text { (SD) }\end{array}$ & $\Delta \log _{10}(95 \% C l)^{a}$ & & \\
\hline \multicolumn{6}{|l|}{ Vaginal } \\
\hline Asymptomatic & 43 & $4.1( \pm 1.1)$ & $\operatorname{Ref}^{b}$ & Ref & Ref \\
\hline Symptomatic & 93 & $4.4( \pm 1.0)$ & $0.28(-0.11-0.66)$ & $0.22(-0.17-0.61)$ & 1.7 \\
\hline \multicolumn{6}{|l|}{ Anorectal } \\
\hline Asymptomatic & 48 & $3.9( \pm 1.2)$ & Ref & Ref & Ref \\
\hline Symptomatic & 4 & $5.4( \pm 0.8)$ & $1.42(0.20-2.65)^{c}$ & $1.67(0.47-2.87)^{d}$ & 46.8 \\
\hline \multicolumn{6}{|l|}{ Oropharyngeal } \\
\hline Asymptomatic & 67 & $2.8( \pm 0.9)$ & Ref & Ref & Ref \\
\hline Symptomatic & 6 & $3.0( \pm 0.9)$ & $0.16(-0.57-0.89)$ & $0.01(-0.69-0.71)$ & 1.0 \\
\hline $\begin{array}{l}\text { a Cl, confidence } \\
\text { b Ref, reference. } \\
\text { c } P<0.05 \text {. } \\
\text { d } P<0.01 \text {. }\end{array}$ & terv & & & & \\
\hline
\end{tabular}


Table 4 Mean N. gonorrhoeae bacterial load and association of symptoms with N. gonorrhoeae bacterial load in heterosexual men

\begin{tabular}{|c|c|c|c|c|c|}
\hline \multirow[b]{2}{*}{ Category } & \multirow[b]{2}{*}{$n$} & \multicolumn{2}{|l|}{ CFU/ml } & \multirow[b]{2}{*}{ Adjusted $\Delta \log _{10}(95 \% \mathrm{Cl})$} & \multirow[b]{2}{*}{$\begin{array}{l}\text { Factor } \\
\text { load }\end{array}$} \\
\hline & & $\begin{array}{l}\text { Mean } \log _{10} \\
\text { (SD) }\end{array}$ & $\Delta \log _{10}(95 \% \mathrm{Cl})^{\mathrm{a}}$ & & \\
\hline \multicolumn{6}{|l|}{ Urine } \\
\hline Asymptomatic & 33 & $3.9( \pm 1.3)$ & $\operatorname{Ref}^{b}$ & Ref & Ref \\
\hline Symptomatic & 78 & $4.7( \pm 0.9)$ & $0.72(0.31-1.14)^{c}$ & $0.74(0.31-1.16)^{c}$ & 5.4 \\
\hline \multicolumn{6}{|l|}{ Oropharyngeal } \\
\hline Asymptomatic & 35 & $3.1( \pm 1.0)$ & $N A^{d}$ & NA & NA \\
\hline Symptomatic & 0 & NA & NA & NA & NA \\
\hline
\end{tabular}

a Cl, confidence interval.

b Ref, reference.

c $P<0.001$.

d NA, not applicable, as no oropharyngeal symptoms were reported.

Table 5 Mean N. gonorrhoeae bacterial load and association of symptoms with N. gonorrhoeae bacterial load in MSM per sample material

\begin{tabular}{|c|c|c|c|c|c|}
\hline \multirow[b]{2}{*}{ Category } & \multirow[b]{2}{*}{$n$} & \multicolumn{2}{|l|}{$\mathrm{CFU} / \mathrm{ml}$} & \multirow[b]{2}{*}{ Adjusted $\Delta \log _{10}(95 \% \mathrm{Cl})$} & \multirow[b]{2}{*}{$\begin{array}{c}\text { Factor } \\
\text { load }\end{array}$} \\
\hline & & $\begin{array}{l}\text { Mean } \log _{10} \\
\text { (SD) }\end{array}$ & $\Delta \log _{10}(95 \% \mathrm{Cl})^{\mathrm{a}}$ & & \\
\hline \multicolumn{6}{|l|}{ Urine } \\
\hline Asymptomatic & 29 & $4.3( \pm 1.0)$ & $\operatorname{Ref}^{b}$ & Ref & Ref \\
\hline Symptomatic & 129 & $4.6( \pm 0.8)$ & $0.36(0.00-0.72)^{c}$ & $0.41\left(0.04-0.77^{c}\right.$ & 2.5 \\
\hline \multicolumn{6}{|l|}{ Anorectal } \\
\hline Asymptomatic & 292 & $4.3( \pm 1.3)$ & Ref & Ref & Ref \\
\hline Symptomatic & 109 & $4.9( \pm 1.2)$ & $0.57(0.29-0.84)^{d}$ & $0.57(0.30-0.85)^{d}$ & 3.8 \\
\hline \multicolumn{6}{|l|}{ Oropharyngeal } \\
\hline Asymptomatic & 260 & $3.1( \pm 1.0)$ & Ref & Ref & Ref \\
\hline Symptomatic & 39 & $3.5( \pm 1.0)$ & $0.34(-0.00-0.67)$ & $0.31(-0.02-0.64)$ & 2.1 \\
\hline
\end{tabular}




\section{Discussion}

This is the first study that determined the $N$. gonorrhoeae bacterial load in a large group of women, heterosexual men, and MSM at three different anatomic sample sites (urogenital, anorectal, and oropharynx). Oropharyngeal swabs had a significantly lower bacterial load (16- to 25-fold) than all other sample materials, whereas the other sample materials had comparable bacterial loads. Also, N. gonorrhoeae bacterial load was comparable in women and men per anatomical sample site. Genital symptoms were associated with a higher $N$. gonorrhoeae bacterial load in urine samples from men but not in vaginal swabs from women. Furthermore, anorectal symptoms were associated with a higher bacterial load in anorectal swabs from both women and MSM, but no difference was seen in oropharyngeal swabs. More than $70 \%$ of $N$. gonorrhoeae infections were single site infections, of which, 31\% were anorectal only and $35 \%$ oropharyngeal only. This implies a need for extragenital testing, at least in MSM. Moreover, $>60 \%$ of $N$. gonorrhoeae infections were asymptomatic, with a wide range between genital (23\% to $32 \%)$, anorectal (75\%), and oropharyngeal (89\%) infections.

We demonstrated that oropharyngeal swabs have on average a 16- to 25 -fold lower bacterial load than all other sample materials, which is in line with previous studies (16, 17). Next to sample material, $N$. gonorrhoeae load was associated with genital symptoms (18-fold) in men and anorectal symptoms in MSM (20-fold) in previous studies $(16,17)$. We observed associations of only 3 - to 5 -fold higher $N$. gonorrhoeae loads in urine samples from men with genital symptoms and 4- to 47-fold higher $N$. gonorrhoeae loads in anorectal swabs from women and MSM with anorectal symptoms. No significant association in women reporting genital symptoms was found, possibly due to the difficulty of recognizing genital symptoms compared to that for men (7). The large difference in results between our study and previous studies could be explained by differences in analytical methods, sample materials, and study population. The strong association of $N$. gonorrhoeae load with symptoms in anorectal swabs from women in our study (47-fold) is likely due to the low number of samples from women reporting anal symptoms $(n=4)$, resulting in a large variation in the estimates (Table 3 ). 
To interpret the $N$. gonorrhoeae load of this study, in our opinion, the only relevant comparison could be with the reported infectious doses of experimental gonococcal infection studies. Hobbs et al. described that a high $N$. gonorrhoeae inoculum load is associated with higher infection probability in experimental gonococcal infections in male volunteers (15). N. gonorrhoeae was inoculated in the urethra by instillation of 0.2 to $0.3 \mathrm{ml} \mathrm{N}$. gonorrhoeae suspension. A $50 \%$ infectious dose $\left(\mathrm{ID}_{50}\right)$, defined as the concentration of an infectious dose of a single exposure needed to infect half of the volunteers, of $3.3 \log _{10}$ CFU for the MS11mkC strain and one of $5 \log _{10}$ CFU for the FA1090 strain were estimated. To compare the N. gonorrhoeae loads of the present study with the reported $I D_{50}$, the $I D_{50}$ dose was divided by the volume of the suspension (average of $0.25 \mathrm{ml}$ ), resulting in $3.9 \log _{10} \mathrm{CFU} / \mathrm{ml}$ for the $\mathrm{MS} 11 \mathrm{mkC}$ strain and $5.6 \log _{10}$ CFU/ml for the FA1090 strain. This would imply that all three anatomical sites have sufficient transmission potential, as the mean N. gonorrhoeae load (3.1 to $4.5 \log _{10}$ CFU/ml) is comparable with the $I_{50}$ doses. It would also mean that asymptomatic $N$. gonorhoeae infections would have a $N$. gonorrhoeae load that is sufficient for transmission, as the mean bacterial load was 2.8 to $4.3 \log _{10}$ CFU/ml in both women and men (Tables 3 to 5). These comparisons and ID50 doses should be interpreted with caution, because the reported inocula of the experimental infection needed for infection varied between studies, most likely because of the inoculation procedures (21). Also, the large difference in $I_{50} s$ between the MS11mkC and FA1090 strains found in the study of Hobbs et al. suggests that infection is not solely driven by bacterial load $(15,22)$. In addition, we determined the N.gonorrhoeae load of an anatomical site, but this does not necessarily reflect the transmitted $N$. gonorrhoeae load per sexual act.

In our study, we observed that $65 \%$ of $N$. gonorrhoeae infections in MSM were single site infections, of which, 33\% were anorectal only and 25\% oropharyngeal only, confirming the need for extragenital testing in MSM. Potentially, we demonstrate the need for testing in women and heterosexual men, as high proportions of oropharyngeal-only infections were found (Table 1). However, women and heterosexual men were not systemically tested at genital and extragenital sites but on indication such as a history of 
direct exposure by anal or oral sex or symptoms. This could introduce bias and elevate the proportion of extragenital-only infections in women and heterosexual men. Moreover, $>60 \%$ of $N$. gonorrhoeae infections are asymptomatic, with a wide range between genital (23\% to $32 \%)$, anorectal $(75 \%)$, and oropharyngeal $(89 \%)$ infections. Asymptomatic infections may potentially lead to unnoticed transmission of $N$. gonorrhoeae and would be unnoticed when routine testing is not applied (as in heterosexual men and in women) (23). Even though the oropharyngeal site might have a lower transmission potential per sexual exposure due to the lower mean N. gonorrhoeae bacterial load, an oropharyngeal $N$. gonorrhoeae infection could have a profound role in transmission $(16,24,25)$. First, oral sex is a common sexual practice in women, heterosexual men, and MSM (11). Second, kissing and saliva use as lubricant have also been proposed as a plausible route of transmission, as substantial amounts of $N$. gonorrhoeae were detected in the saliva of $\operatorname{MSM}(24,26)$. In addition, oropharyngeal infections are thought to have a key role in the AMR development in $N$. gonorrhoeae because of the common presence of commensal Neisseria species that readily exchange DNA (14). As the estimated median duration of oropharyngeal $N$. gonorrhoeae infection is at least 15 weeks, it could be a reservoir for onward transmission and antimicrobialresistant strains $(14,27,28)$.

One of the strengths of this study is the diversity of the study population, achieved by including women and heterosexual men along with MSM and the routine extragenital testing in MSM. One of its weaknesses is that women and heterosexual men were not routinely tested extragenitally, thereby potentially underestimating the relative number of extragenital N. gonorrhoeae infections. Generalizability might be difficult, as only highrisk individuals for acquiring an STI were studied (10). In addition, the volume of firstvoid urine taken per consultation can vary, and that could have an impact on the determined $N$. gonorrhoeae bacterial load in a sample. This could also explain the large range of observed bacterial load in urine samples in Fig. 1. 
In conclusion, our results suggest that all anatomical sample sites may have a role in transmission because of the substantial $N$. gonorrhoeae load. Oropharyngeal $N$. gonorrhoeae infections might have a smaller role, as the mean bacterial load is lower. It would also imply that a N. gonorrhoeae infection is equally relevant in women and men, as there is no difference in bacterial load. Oropharyngeal infections should be monitored, since they are frequently single infections, mostly asymptomatic, and could be involved in the AMR development of $N$. gonorrhoeae. Furthermore, extragenital testing should be systematically implemented in routine diagnostics, at least in MSM, to reduce missed infections and help improve N. gonorrhoeae control. 


\section{References}

1. Rowley J, Vander Hoorn S, Korenromp E, Low N, Unemo M, Abu-Raddad LJ, Chico RM, Smolak A, Newman L, Gottlieb S, Thwin SS, Broutet N, Taylor MM. 2019. Chlamydia, gonorrhoea, trichomoniasis and syphilis: global prevalence and incidence estimates, 2016. Bull World HealthOrgan 97:548P-562P. https://doi.org/10.2471/BLT.18.228486.

2. World Health Organization. 2012. Global action plan to control the spread and impact of antimicrobial resistance in Neisseria gonorrhoeae. World Health Organization, Geneva, Switzerland.

3. Centers for Disease Control and Prevention. 2014. Sexually transmitted disease surveillance 2014. Centers for Disease Control and Prevention, Atlanta, GA.

4. Reed JL, Huppert JS, Gillespie GL, Taylor RG, Holland CK, Alessandrini EA, Kahn JA. 2015. Adolescent patient preferences surrounding partner notification and treatment for sexually transmitted infections. Acad Emerg Med 22:61-66. https://doi.org/10.1111/acem.12557.

5. Theunissen KA, Bos AE, Hoebe CJ, Kok G, Vluggen S, Crutzen R, Dukers-Muijrers NH. 2015. Chlamydia trachomatis testing among young people: what is the role of stigma? BMC Public Health 15:651. https://doi.org/10.1186/s12889-015-2020-y.

6. Bignell C, Unemo M, European STI Guidelines Editorial Board. 2013. 2012 European guideline on the diagnosis and treatment of gonorrhoea in adults. Int J STD AIDS 24:85-92. https://doi.org/10.1177/0956462412472837.

7. Workowski KA, Bolan GA, Centers for Disease Control and Prevention. 2015. Sexually transmitted diseases treatment guidelines, 2015. MMWR Recomm Rep 64:1-137.

8. Centers for Disease Control and Prevention. 2014. Recommendations for the laboratory-based detection of Chlamydia trachomatis and Neisseria gonorrhoeae-2014. MMWR Recomm Rep 63:119.

9. LeFevre ML, U.S. Preventive Services Task Force. 2014. Screening for chlamydia and gonorrhea: U.S. Preventive Services Task Force recommendation statement. Ann Intern Med 161:902-910. https://doi.org/10.7326/M14-1981.

10. Dukers-Muijrers NH, Schachter J, van Liere GA, Wolffs PF, Hoebe CJ. 2015. What is needed to guide testing for anorectal and pharyngeal Chlamydia trachomatis and Neisseria gonorrhoeae in women and men? Evidence and opinion. BMC Infect Dis 15:533. https://doi.org/10.1186/s12879-015-1280-6.

11. Garner AL, Schembri G, Cullen T, Lee V. 2015. Should we screen heterosexuals for extra-genital chlamydial and gonococcal infections? Int J STD AIDS 26:462- 466.

https://doi.org/10.1177/0956462414543120.

12. Koedijk FD, van Bergen JE, Dukers-Muijrers NH, van Leeuwen AP, Hoebe CJ, van der Sande MA, Dutch STIC, Dutch STI centres. 2012. The value of testing multiple anatomic sites for gonorrhoea and chlamydia in sexually transmitted infection centres in the Netherlands, 2006-2010. Int J STD AIDS 23:626-631. https://doi.org/10.1258/ijsa.2012.011378.

13. Chan PA, Janvier M, Alexander NE, Kojic EM, Chapin K. 2012. Recommendations for the diagnosis of Neisseria gonorrhoeae and Chlamydia trachomatis, including extra-genital sites. Med Health R I 95:252-254.

14. Unemo M, Shafer WM. 2014. Antimicrobial resistance in Neisseria gonorrhoeae in the 21st century: past, evolution, and future. Clin Microbiol Rev 27:587-613. https://doi.org/10.1128/CMR.00010-14.

15. Hobbs MM, Sparling PF, Cohen MS, Shafer WM, Deal CD, Jerse AE. 2011. Experimental gonococcal infection in male volunteers: cumulative experience with Neisseria gonorrhoeae strains FA1090 and MS11mkC. Front Microbiol 2:123. https://doi.org/10.3389/fmicb.2011.00123.

16. Bissessor M, Tabrizi SN, Fairley CK, Danielewski J, Whitton B, Bird S, Garland S, Chen MY. 2011. Differing Neisseria gonorrhoeae bacterial loads in the pharynx and rectum in men who have sex with men: implications for gonococcal detection, transmission, and control. J Clin Microbiol 49:43044306. https://doi.org/10.1128/JCM.05341-11.

17. Priest D, Ong JJ, Chow EP, Tabrizi S, Phillips S, Bissessor M, Fairley CK, Bradshaw CS, Read TR, Garland S, Chen M. 2017. Neisseria gonorrhoeae DNA bacterial load in men with symptomatic and asymptomatic gonococcal urethritis. Sex Transm Infect 93:478-481. https://doi.org/10.1136/sextrans-2016-052950.

18. National Institute for Public Health and the Environment. 2018. Draaiboek Consult seksuele gezondheid. National Institute for Public Health and the Environment, Bilthoven, the Netherlands. 
19. Parra-Sánchez M, Palomares JC, Bernal S, González MT, Sivianes N, Pérez L, Pueyo I, Martín-Mazuelos E. 2012. Evaluation of the cobas 4800 CT/NG Test for detecting Chlamydia trachomatis and Neisseria gonorrhoeae DNA in urogenital swabs and urine specimens. Diagn Microbiol Infect Dis 74:338 -342. https://doi.org/10.1016/j.diagmicrobio.2012.08.004.

20. Marlowe EM, Hardy D, Krevolin M, Gohl P, Bertram A, Arcenas R, Seiverth B, Schneider T, Liesenfeld O. 2017. High-throughput testing of urogenital and extragenital specimens for detection of Chlamydia Trachomatis and Neisseria Gonorrhoeae with Cobas CT/NG. Eur J Microbiol Immunol (Bp) 7:176-186. https://doi.org/10.1556/1886.2017.00018.

21. Cohen MS, Cannon JG, Jerse AE, Charniga LM, Isbey SF, Whicker LG. 1994. Human experimentation with Neisseria gonorrhoeae: rationale, methods, and implications for the biology of infection and vaccine development. J Infect Dis 169:532-537. https://doi.org/10.1093/infdis/169.3.532.

22. Schmidt KA, Schneider H, Lindstrom JA, Boslego JW, Warren RA, Van de Verg L, Deal CD, McClain JB, Griffiss JM. 2001. Experimental gonococcal urethritis and reinfection with homologous gonococci in male volunteers. Sex Transm Dis 28:555-564. https://doi.org/10.1097/00007435-200110000-00001.

23. Whittles LK, White PJ, Didelot X. 2017. Estimating the fitness cost and benefit of cefixime resistance in Neisseria gonorrhoeae to inform prescription policy: a modelling study. PLoS Med 14:e1002416. https://doi.org/10.1371/journal.pmed.1002416.

24. Fairley CK, Cornelisse VJ, Hocking JS, Chow EPF. 2019. Models of gonorrhea transmission from the mouth and saliva. Lancet Infect Dis 19:E360 -E366. https://doi.org/10.1016/S1473-3099(19)30304-4.

25. van Liere G, Dukers-Muijrers N, Wessel SK, Gotz HM, Hoebe C. 26 September 2019. What is the optimal testing strategy for oropharyngeal Neisseria gonorrhoeae in men who have sex with men? Comparing selective testing versus routine universal testing from Dutch STI clinic data (2008 -2017). Clin Infect Dis https://doi.org/10.1093/cid/ciz964.

26. Chow EP, Tabrizi SN, Phillips S, Lee D, Bradshaw CS, Chen MY, Fairley CK. 2016. Neisseria gonorrhoeae bacterial DNA load in the pharynges and saliva of men who have sex with men. J Clin Microbiol 54:2485-2490. https://doi.org/10.1128/JCM.01186-16.

27. Chow EP, Camilleri S, Ward C, Huffam S, Chen MY, Bradshaw CS, Fairley CK. 2016. Duration of gonorrhoea and chlamydia infection at the pharynx and rectum among men who have sex with men: a systematic review. Sex Health 13:199 -204. https://doi.org/10.1071/SH15175.

28. Barbee L, Soge O, Haglund M, Yeung W, Hughes J, Khosropour C, Golden M. 2019. O02.4 Incidence and duration of pharyngeal and rectal gonorrhea and chlamydia among high-risk men who have sex with men (MSM). Sex Transm Infect 95 (Suppl 1):A40.2-A40. 


\section{Supplemental files}

1480 NG positive samples

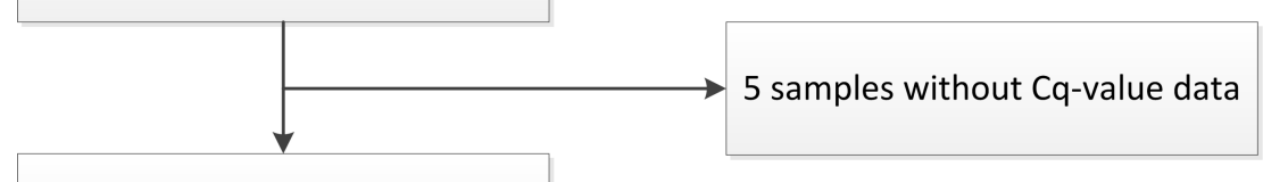

1475 samples with Cq-value data

7 samples without data on sexual risk group

1468 samples with data on sexua risk group

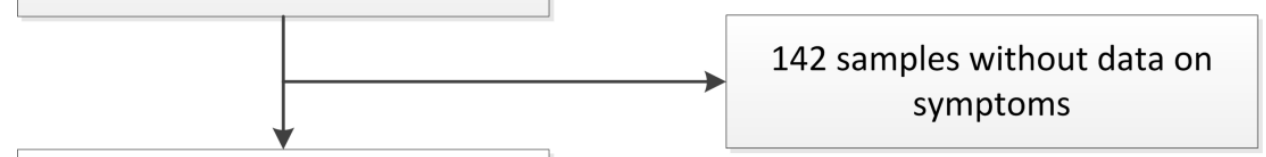

1326 samples with data on symptoms

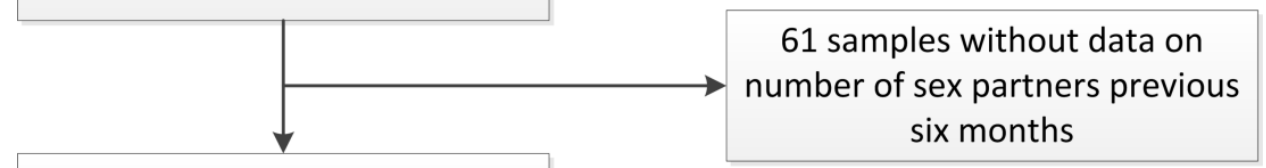

1265 samples available for analysis

Supplemental figure 1 Flowchart, including Neisseria gonorrhoeae positive samples for analysis 
Supplemental table 1 Mean NG bacterial load and association of symptoms with NG bacterial load in women per sample material. Women included in the analysis (97/197) were tested at genital and extra-genital sites. NG load of NG negative samples was set at 0 .

\begin{tabular}{|c|c|c|c|c|c|c|}
\hline Women & $n$ & $\begin{array}{l}\text { Mean } \log _{10} \\
\text { CFU/mL } \\
\text { (SD) }\end{array}$ & $\begin{array}{l}\Delta \log _{10} \\
\text { CFU/mL }(95 \% \mathrm{Cl})\end{array}$ & $\begin{array}{l}\text { Adjusted } \Delta \log _{10} \\
\text { CFU/mL }(95 \% C l)\end{array}$ & $\begin{array}{c}\text { Adjusted } \Delta \log _{10} \\
\text { CFU/mL }(95 \% \mathrm{Cl}) \\
\text { including NG } \\
\text { load concurrent } \\
\text { site }\end{array}$ & $\begin{array}{c}\text { Factor } \\
\text { load }\end{array}$ \\
\hline \multicolumn{7}{|l|}{ Vaginal } \\
\hline Asymptomatic & 17 & $3.7( \pm 1.3)$ & Ref & Ref & Ref & Ref \\
\hline Symptomatic & 28 & $4.3( \pm 1.0)$ & $0.58(-0.11-1.27)$ & $0.48(-0.22-1.19)$ & $0.60(-0.11-1.31)$ & 4.0 \\
\hline \multicolumn{7}{|l|}{ Anorectal } \\
\hline Asymptomatic & 39 & $4.0( \pm 1.2)$ & Ref & Ref & Ref & Ref \\
\hline Symptomatic & 1 & 5.7 & $1.74(-0.70-4.18)$ & $2.01(-0.22-4.25)$ & $1.91(-0.42-4.25)$ & 82.1 \\
\hline \multicolumn{7}{|l|}{ Oropharyngeal } \\
\hline Asymptomatic & 56 & $2.8( \pm 0.9)$ & Ref & Ref & Ref & Ref \\
\hline Symptomatic & 6 & $3.0( \pm 0.9)$ & $0.17(-0.57-0.91)$ & $-0.05(-0.76-0.67)$ & $-0.06(-0.81-0.69)$ & 0.9 \\
\hline
\end{tabular}

Abbreviations: CFU, Colony forming units; SD, standard deviation, $\mathrm{Cl}$, confidence interval, Ref, reference 
Supplemental table 2 Mean NG bacterial load and association of symptoms with NG bacterial load in MSM per sample material. MSM included in the analysis $(595 / 603)$ were tested at genital and extra-genital sites. NG load of NG negative samples was set at 0 .

\begin{tabular}{|c|c|c|c|c|c|c|}
\hline MSM & $n$ & $\begin{array}{l}\text { Mean } \\
\log _{10} \\
\text { CFU/mL } \\
\text { (SD) }\end{array}$ & $\begin{array}{l}\Delta \log _{10} \\
\text { CFU/mL }(95 \% \mathrm{Cl})\end{array}$ & $\begin{array}{l}\text { Adjusted } \Delta \log _{10} \\
\text { CFU/mL }(95 \% \mathrm{Cl})\end{array}$ & $\begin{array}{c}\text { Adjusted } \Delta \log _{10} \\
\text { CFU/mL }(95 \% \mathrm{Cl}) \\
\text { including NG load } \\
\text { concurrent site }\end{array}$ & $\begin{array}{c}\text { Factor } \\
\text { load }\end{array}$ \\
\hline \multicolumn{7}{|l|}{ Urine } \\
\hline Asymptomatic & 29 & $4.3( \pm 1.0)$ & Ref & Ref & Ref & Ref \\
\hline Symptomatic & 128 & $4.6( \pm 0.8)$ & $0.36(-0.00-0.72)$ & $0.41(0.04-0.77)^{*}$ & $0.40(0.03-0.77)^{*}$ & 2.5 \\
\hline \multicolumn{7}{|l|}{ Anorectal } \\
\hline Asymptomatic & 288 & $4.3( \pm 1.3)$ & Ref & Ref & Ref & Ref \\
\hline Symptomatic & 106 & $4.9( \pm 1.2)$ & $0.58(0.30-0.86)^{* * * *}$ & $0.59(0.31-0.87)^{* * * *}$ & $0.56(0.28-0.83)^{* * * *}$ & 3.6 \\
\hline \multicolumn{7}{|l|}{ Oropharyngeal } \\
\hline Asymptomatic & 256 & $3.1( \pm 1.0)$ & Ref & Ref & Ref & Ref \\
\hline Symptomatic & 36 & $3.5( \pm 1.0)$ & $0.33(-0.02-0.68)$ & $0.30(-0.04-0.64)$ & $0.31(-0.03-0.65)$ & 2.1 \\
\hline
\end{tabular}

${ }^{*}=p<0.05 ;{ }^{* * *}=p<0.001$

Abbreviations: CFU, Colony forming units; SD, standard deviation, $\mathrm{Cl}$, confidence interval, Ref, reference 


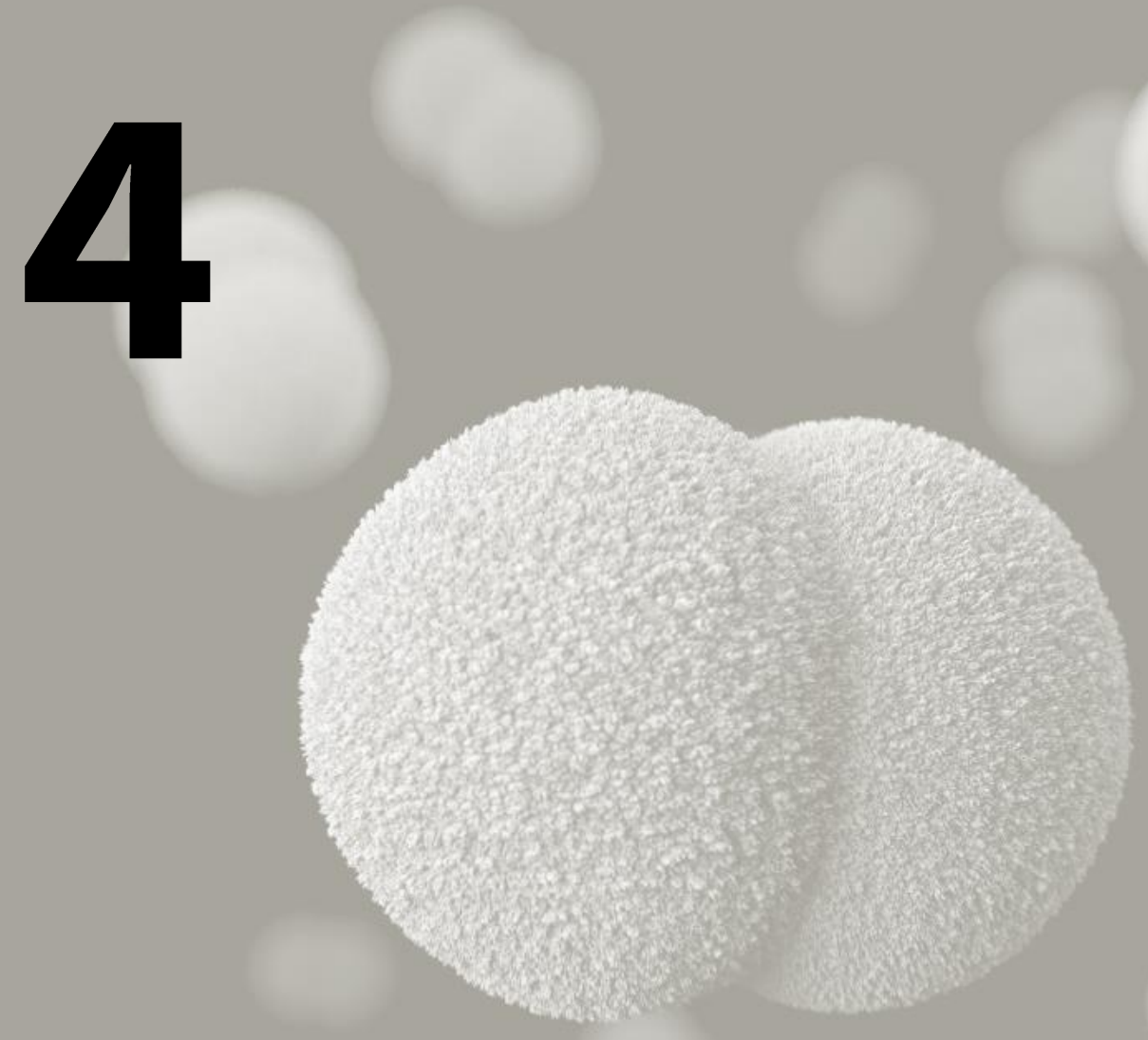


Culture-free genotyping of Neisseria gonorrhoeae revealed distinct strains at different anatomical sites in a quarter of patients, the Netherlands, 2012 to 2016

Brian M.J.W. van der Veer, Petra F.G. Wolffs, Christian J.P.A. Hoebe,

Nicole H.T.M. Duker-Muijrers, Lieke B. van Alphen Eurosurveillance. 2018 doi: 10.2807/1560-7917.ES.2018.23.50.1800253 


\section{Abstract}

Background: Genotyping of Neisseria gonorrhoeae (NG) is essential for surveillance to monitor NG transmission and dissemination of resistant strains. Current genotyping methods rely on bacterial culture which frequently fails.

Aim: Our aim was to develop a culture-free genotyping method that is compatible with the widely used $N$. gonorrhoeae multi-antigen sequence typing (NG-MAST) database, which facilitates genotyping of NG detected at separate anatomical sites in individual patients.

Methods: Specific primers for both $P C R$ targets por $B$ and tbp $B$ were designed and technically validated by assessing the analytical sensitivity, cross-reactivity with 32 nongonoccocal Neisseria species, and concordance with NG-MAST. Clinical application was assessed on 205 paired samples from concurrent NG infections at different anatomical sites of 98 patients ( 81 men who have sex with men and 17 women) visiting our sexually transmitted infections clinic.

Results: Typing could be consistently performed on samples with a PCR quantification cycle $(\mathrm{Cq})$ value $<35$. Furthermore, the method showed no cross-reactivity and was concordant with NG-MAST. Culture-free NG-MAST improved the typing rate from $62 \%$ (59/95) for cultured samples to $94 \%$ (89/95) compared with culture-dependent NGMAST. Paired samples of 80 of 98 patients were genotyped, revealing distinct NG strains in separate anatomical sites in $25 \%(20 / 80)$ of the patients.

Conclusions: This NG-specific genotyping method can improve NG surveillance as it facilitates genotyping of non-culturable and extra-genital samples. Furthermore, $25 \%$ of patients were infected with multiple NG strains, which is missed in current culturedependent surveillance. Including non-culturable and concurrent NG infections in surveillance informs actions on dissemination of multidrug-resistant NG strains. 


\section{Introduction}

Neisseria gonorrhoeae (NG) is one of the most common bacterial sexually transmitted infections [1]. The World Health Organization (WHO) estimates that more than 100 million new cases of NG occur each year, even though testing for NG and diagnostics have improved $[1,2]$. Detection of NG allows empirical treatment that results in cure in at least $95 \%$ of cases, and rapid cure subsequently limits transmission [1]. However, increasingly resistant strains of NG have been reported in the last decades, which could complicate empirical treatment [3]. Therefore, gaining insight in transmission and antimicrobial resistance (AMR) of NG is important. NG can be detected by culture or nucleic acid amplification test (NAAT) but both methods have limitations [2]. Culture is known to be less sensitive because NG requires demanding nutritional and environmental conditions, leading to a low percentage of culture-confirmed diagnoses [4]. In contrast, NAAT are more sensitive but cannot determine the AMR profile [2,5].

Surveillance of NG is essential to monitor transmission and dissemination of resistant strains. NG multi-antigen sequence typing (NG-MAST) is a widely used genotyping method to monitor transmission and outbreaks [6,7]. This method has a higher discriminatory power than multilocus sequence typing (MLST) and is more cost-effective than highly discriminatory whole genome sequencing $[8,9]$. In addition, some of the NGMAST sequence types (ST) are associated with AMR $[7,10]$. The currently used NG-MAST protocol requires culture because the primers cross-react with other Neisseria species [11]. To date, only two studies have genotyped non-cultured clinical samples with NGMAST $[6,11]$. Whiley et al. demonstrated that NG-MAST can be applied to non-cultured urogenital samples but not to samples from extra-genital sites (oropharynx and rectum) because of the presence of commensal Neisseria species [11]. They showed that mainly N. lactamica, N. meningitidis and N. polysaccharea strains lead to the cross-reactivity. Furthermore, it appeared that successful application of NG-MAST to non-cultured samples was linked to the quantification cycle (Cq) of the PCR-positive sample because four of the five failed samples had a high Cq value (>35). 
Previous studies have shown that patients can be NG-positive at extra-genital sites and have concurrent NG infections at different anatomical sites [12,13]. Most of the extragenital and concurrent infections are observed in risk groups, for example in men who have sex with men (MSM). Extra-genital sites may act as a reservoir for AMR genes as the present commensal Neisseria species, potentially harbouring AMR genes, readily exchange DNA with NG [14]. Typing the oropharyngeal site using culture-dependent methods is especially difficult because the bacterial load is lower than at other anatomical sites and this appears to be linked to culture success [15]. In previous studies, concurrent NG infections were studied with various genotyping methods [6,11,16-18]. Distinct NG strains per anatomical site have been observed and some strains demonstrated discordant antibiotic susceptibility profiles [6,16-18]. The observed distinct NG strains could be explained by high-risk sexual behaviour and patients being part of different transmission chains $[16,18]$. However, these studies were small (fewer than 10 patients), focussed on cultured isolates, used a single-position single nucleotide polymorphisms (SNP) and/or used non NG-specific primers [6,16-18]. Therefore, we aimed to develop a culture-free NG-MAST genotyping method that does not cross-react with other Neisseria species and is compatible with the NG-MAST database. Furthermore, we aimed to gain more insight in the frequency of distinct NG strains at separate anatomical sites in individual patients.

\section{Methods}

This study was designed to test the clinical application of the culture-free NG-MAST method to non-culturable clinical samples and use these data to compare ST of separate anatomical sites within a patient. The method was technically validated by assessing analytical sensitivity, specificity and concordance with NG-MAST.

\section{Clinical samples}

All NG-positive clinical samples $(n=1,110)$ from different anatomical sites were retrieved from 814 consultations (further referred to as number of patients) from 642 individual patients. NG positivity was based on NG detection by the Cobas 4800 CT/NG NAAT assay (Roche Diagnostics, Basel, Switzerland), between January 2012 and May 2016 from our 
sexually transmitted infections (STI) clinic (South Limburg Public Health Service). These samples were from MSM ( $n=769$ samples), women $(n=254$ samples) and heterosexual men ( $n=87$ samples). Samples with a Cq value of 35 or higher did not consistently yield PCR products in dilution series (see technical validation). Therefore, clinical samples with a Cq value of $\geq 35$ were excluded $(n=418)$, leaving 692 samples for analysis. Of the remaining 692 NG-positive samples, we included only paired samples from separate anatomical sites belonging to a single STI clinic visit of a patient $(n=228)$. Different pairs of any combination of genital, anorectal or oropharyngeal NG positivity were observed. A total of 108 patients were NG-positive at two or three anatomical sites (90 MSM and 18 women who reported anal sex or symptoms and who were systematically tested on all three anatomical sites). The remaining amount of sample material was not sufficient for typing for 10 patients (nine MSM and one woman) and therefore these patients were excluded, leaving 98 patients with paired samples for analysis. In total, 205 Cobas NAAT clinical samples were included: 57 urine, 17 vaginal, 92 anorectal and 39 oropharyngeal samples. With these samples, we assessed the clinical application of the culture-free NGMAST method and the presence of distinct STs within a patient. Data on culture success were retrieved by routine diagnostics because NG culture is mostly performed as part of the national NG resistance surveillance since NAAT diagnosis of NG is the primary diagnostic procedure. All patients were treated with a single dose of ceftriaxone, the primary choice of treatment because no resistance exists in the Netherlands [4]. An additional swab or urine sample for routine NG culture is taken at the treatment visit at the STI clinic only when treatment has not already been provided at the diagnostic visit based on symptoms. For this study, data of this routine culture was available for all patients if culture was performed.

\section{DNA isolation clinical samples}

Total DNA was isolated from $400 \mu \mathrm{L}$ Cobas 4800 clinical samples using the QIAamp DNA Mini Kit (Qiagen, Hilden, Germany) and eluted in $50 \mu \mathrm{L}$ Milli-Q water (MQ). To increase elution yield, we extended the incubation time to $10 \mathrm{~min}$. The eluate was stored at $-20^{\circ} \mathrm{C}$. 


\section{DNA isolation cultured gonoccocal and non-gonoccocal Neisseria strains}

Gonoccocal and non-gonoccocal clinical and reference Neisseria strains were inoculated on chocolate agar with IsoVitaleX or blood agar (BectonDickinson, Sparks, United States (US)) and incubated over night at $37{ }^{\circ} \mathrm{C}$ in $5 \% \mathrm{CO}_{2}$. Morphology of the colonies was checked and a single colony was subcultured before DNA isolation. Bacterial suspensions were prepared in sterile saline solution from two or three colonies (depending on the size of the colonies) picked with a pre-wetted sterile swab. The bacteria were pelleted by centrifugation at $2,000 \mathrm{~g}$ for $5 \mathrm{~min}$ and washed once. The pellet was resuspended in $500 \mu \mathrm{L} \mathrm{MQ}$ and boiled for $10 \mathrm{~min}$. Cell debris was pelleted by centrifugation at $8,000 \mathrm{~g}$ for $2 \mathrm{~min}$ and the supernatant was stored at $-20^{\circ} \mathrm{C}$.

\section{NG-MAST genotyping}

PCR for both targets was performed in $50 \mu \mathrm{L}$ reaction volumes using the Biometra T3000 Thermal Cycler (Labrepco Inc., US). Each reaction per target (porB and tbpB) contained 50 pmol of the NG-MAST forward and reverse primer for the respective target (Table 1), 2.5 U HotStar polymerase (Qiagen), 1× Qiagen PCR buffer, $0.2 \mathrm{mmol} / \mathrm{L}$ dNTP, $5 \mu \mathrm{L}$ DNA lysate and MQ to a volume of $50 \mu \mathrm{L}$. The PCR protocol of Martin et al. was used to amplify the targets but cycles were increased to 30 [7].

Table 1. Overview of primers used in PCR and sequencing reactions for NG-MAST and culture-free NG-MAST

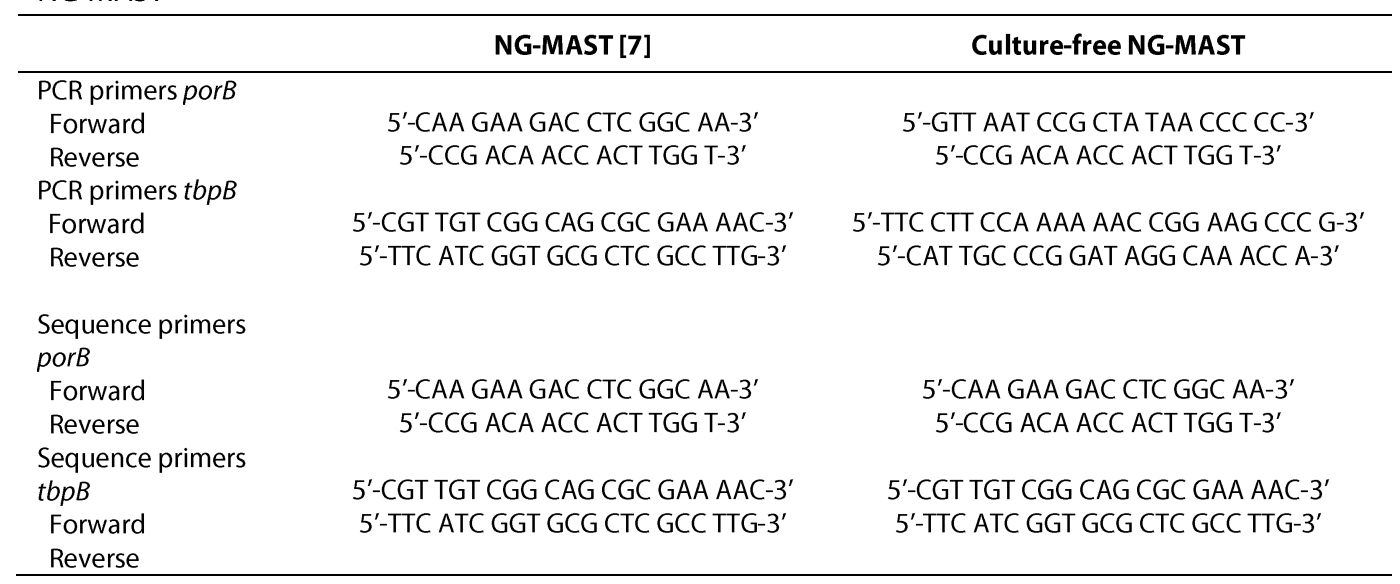


The amplicons were precipitated with $50 \mu \mathrm{L} 20 \%$ polyethylene glycol 8000 and $2.5 \mathrm{~mol} / \mathrm{L}$ sodium chloride at $37^{\circ} \mathrm{C}$ for $15 \mathrm{~min}$. Precipitated amplicons were centrifuged at $15,000 \times \mathrm{g}$ for $15 \mathrm{~min}$ and washed twice with ice-cold $80 \%$ ethanol. The pellet was allowed to dry and resuspended in $25 \mu \mathrm{L} \mathrm{MQ}$. The porB and tbpB fragments were sequenced with their respective forward and reverse primer using the BigDye Terminator v1.1 Cycle Sequencing kit (Thermo Fisher Scientific, Waltham, Massachusetts, US). The sequence protocol has an initial denaturation step of 1 min at $96^{\circ} \mathrm{C}$, followed by 25 cycles of $10 \mathrm{~s}$ at $96^{\circ} \mathrm{C}, 10 \mathrm{~s}$ at $55^{\circ} \mathrm{C}($ por $B)$ or $65^{\circ} \mathrm{C}\left(\right.$ tbp B) , and 3 min at $60^{\circ} \mathrm{C}$.

\section{Primer design culture-free NG-MAST}

The genome sequences of all NG reference strains published by the WHO $(n=14)$ were downloaded from GenBank and used for multiple alignments with Clustal Omega [19]. A $2 \mathrm{~kb}$ flanking region of the aligned NG-MAST porB and $t b p B$ primers were selected to identify conserved regions. Each flanking region was aligned and conserved regions were tested for in silico specificity using basic-local alignment search tool (BLAST). A specific sequence was identified that could be used as the forward primer for porB but no specific sequence was identified for the reverse primer, therefore the NG-MAST reverse primer was used which resulted in a fragment of ca $1.2 \mathrm{~kb}$ (Table 1). Two specific sequences were identified for $t b p B$ which could be used as a forward and reverse primer, resulting in a fragment of ca $1.8 \mathrm{~kb}$ (Table 1).

\section{Culture-free NG-MAST genotyping}

This method was similar to the NG-MAST method apart from the initial PCR. Each reaction per target (porB and tbpB) contained $50 \mathrm{pmol}$ of the culture-free NG-MAST forward and reverse primer for the respective target (Table 1), $0.2 \mu \mathrm{L}$ AccuPrime Taq DNA Polymerase High Fidelity (Thermo Fisher), 1× AccuPrime PCR buffer II, $15 \mu \mathrm{L}$ DNA isolated from a clinical sample and MQ to a volume of $50 \mu \mathrm{L}$. The PCR protocol had an initial denaturation step of $5 \mathrm{~min}$ at $95^{\circ} \mathrm{C}$, followed by 40 cycles of $30 \mathrm{~s}$ at $95^{\circ} \mathrm{C}, 60 \mathrm{~s}$ at $58^{\circ} \mathrm{C}$ (porB) or $69^{\circ} \mathrm{C}(\operatorname{tbp} B), 2.5 \mathrm{~min}$ at $68^{\circ} \mathrm{C}$, and a final extension of $10 \mathrm{~min}$ at $68^{\circ} \mathrm{C}$. The porB and tbpB amplicons were sequenced with NG-MAST primers (Table 1). The culturefree method was therefore compatible with the NG-MAST online database because we 
characterised the same fragments of $p o r B$ and $t b p B$ genes.

\section{Technical validation of culture-free NG-MAST method}

Analytical sensitivity was determined using dilution series ranging from $1.3 \times 10^{6}$ to $1.3 \times 10^{2}$ colony-forming units (CFU)/mL. Concordance of culture-free NG-MAST method with NG-MAST was tested with seven randomly selected isolates cultured from four urine samples, two anorectal swabs and one oropharyngeal swab, and their respective unculturable Cobas 4800 screening samples between January and March 2017. The isolates were subjected to NG-MAST genotyping whereas the clinical samples were subjected to the culture-free NG-MAST method. The analytical specificity was tested with a panel of 32 non-gonococcal Neisseria species strains, including $N$. cinerea $(n=1)$, $N$. denitrificans $(n=1), N$. elongata $(n=1), N$. flavescens $(n=1), N$. lactamica $(n=2), N$. meningitidis $(n=3), N$. mucosa $(n=7), N$. perflava $(n=1), N$. polysaccharea $(n=1)$ and $N$. subflava $(n=14)$.

\section{Data analysis}

The trace files were assembled, trimmed and edited using Bionumerics (version 7.6, Applied Maths, Sint-Martens-Latem, Belgium). The starting trimming patterns for por $B$ and $t b p B$ and lengths were used as described in Martin et al. [7]. Alleles and ST were called according to the NG-MAST online curated database. Phylogenetic trees of porB, $t b p B$ and concatenated sequences were constructed using multiple alignment and unweighted pair group method with arithmic mean (UPGMA) clustering using default settings with gap penalty at $100 \%$.

\section{Ethical statement}

The study protocol was approved as a scientific study not done in humans by the Medical Ethical Committee of Maastricht University Medical Centre (MUMC+; number METC 2017-2-0251) as it concerned a laboratory and observational study using anonymous data and leftover diagnostic samples only. This was part of an STI clinic procedure where patients did not object to the use of their data and samples anonymously for research purposes. 


\section{Results}

\section{Analytical sensitivity, specificity and concordance of culture-free NG-MAST}

Dilution series in triplicate showed that culture-free NG-MAST consistently yielded PCR products for both porB and $t b p B$ in samples with a Cq value $<35$. None of the tested 32 non-gonococcal Neisseria strains were PCR-positive for either porB or $t b p B$ in the culturefree NG-MAST PCR reactions. The seven randomly selected cultured isolates had identical ST as their respective unculturable Cobas 4800 screening sample but distinct ST were observed between the selected isolates.

\section{Paired clinical samples}

In total, $90.2 \%(185 / 205)$ of the selected paired clinical samples were successfully genotyped with the culture-free NG-MAST method. The porB fragment was successfully sequenced in 95.6\% (196/205) of samples and tbpB in 93.7\% (192/205). Failure of both targets in a sample does not appear to be related to the Cq value because both low $(<30)$ and higher (30-35) Cq values show comparable failure rates (data not shown). We observed 36 different porB and 22 tbpB alleles, resulting in 45 ST. Among the samples, porB-1808 and $t b p B-29$ were the most common alleles, present in 51 and 49 samples, respectively. Furthermore, we found five previously unidentified $p o r B$ and two tbpB alleles which all had the highest identity with NG using a BLAST search. The most prevalent ST were ST2992 ( $n=36)$, ST11461 $(n=30)$, and ST5441 $(n=26)$, and 15 new STs were found.

Routine culture was performed for 95 of the 205 paired clinical samples and 59 (62.1\%) were culture-positive. Typically, only one anatomical site was sampled for culture, and the majority of the culture-positive samples were collected from the genital site (44/59). Culture-free NG-MAST applied to the non-culturable clinical material (Cobas 4800 sample material) of samples send in for culture (including culture-negative samples) showed that $93.7 \%$ (89/95) were genotyped successfully. However, four samples negative in culture-free NG-MAST were culture-positive. Of the remaining 110 uncultured clinical samples, 98 (89.1\%) could be genotyped. 


\section{Sequence diversity within porB and tbpB alleles}

High sequence diversity was observed for both $\operatorname{por} B$ and $t b p B$ in this study population (Supplement Figures S1 and S2). Two porB alleles (90 and 2723) were divergent, with more than $50 \%$ dissimilarity, from all other observed alleles. The newly identified $t b p B$ allele with $91 \%$ similarity with $t b p B-1251$ was divergent from all other observed alleles with more than $60 \%$ dissimilarity. In addition, the average dissimilarity between tbpB alleles appeared to be greater than between porB alleles.

\section{Sequence types of samples from separate anatomical sites in a patient}

In this dataset of clinical samples, we genotyped 169 paired samples (taken from a single patient at separate anatomical sites) from 80 patients (66 MSM and 14 women) (Supplement Table). We observed distinct concurrent ST in a quarter (20/80) of the patients. They had the following combinations of sample material: urine-anorectal $(n=6)$, urine-oropharyngeal $(n=1)$, anorectal-oropharyngeal $(n=8)$, urine-anorectaloropharyngeal $(n=1)$, vaginal-anorectal $(n=3)$, and vaginal-anorectal-oropharyngeal $(n=1)$ (Table 2). Similar proportions of distinct concurrent ST were observed in MSM $(16 / 66)$ and women (4/14). Interestingly, a single patient (patient 32) was NG-positive with a distinct NG strain at all three tested anatomical sites (Table 2). 
Table 2. Characteristics of patients with concurrent Neisseria gonorrhoeae infection with distinct sequence types, including age, risk group, multiple sequence types and NG-MAST results per sample site, the Netherlands, January 2012-May $2016(n=42)$

\begin{tabular}{|c|c|c|c|c|c|c|c|c|c|c|c|c|c|c|}
\hline \multirow{2}{*}{ Patient } & \multirow{2}{*}{$\begin{array}{c}\text { Age } \\
\text { (years) }\end{array}$} & \multirow{2}{*}{$\begin{array}{c}\text { Risk } \\
\text { group }\end{array}$} & \multicolumn{3}{|c|}{ Urine } & \multicolumn{3}{|c|}{ Vaginal } & \multicolumn{3}{|c|}{ Anorectal } & \multicolumn{3}{|c|}{ Oropharyngeal } \\
\hline & & & porB & tbpB & ST & porB & tbpB & ST & porB & tbpB & ST & porB & tbpB & ST \\
\hline 9 & 26 & Women $^{\mathrm{a}}$ & - & - & - & 1808 & $\begin{array}{l}91 \%- \\
1251\end{array}$ & $\begin{array}{l}\text { New } \\
\text { ST1 }\end{array}$ & 1808 & 29 & 2992 & - & - & - \\
\hline 10 & 22 & MSM & - & - & - & - & - & - & 3031 & 33 & 4995 & 1489 & 33 & 10257 \\
\hline 24 & 27 & MSM & - & - & - & - & - & - & 7272 & 33 & $\begin{array}{l}\text { New } \\
\text { ST4 }\end{array}$ & $\begin{array}{l}99 \%- \\
7988\end{array}$ & 110 & $\begin{array}{l}\text { New } \\
\text { ST3 }\end{array}$ \\
\hline 27 & 24 & Women $^{a}$ & - & - & - & 301 & 29 & 359 & 2723 & 110 & 4431 & 301 & 29 & 359 \\
\hline 28 & 20 & MSM & - & - & - & - & - & - & 908 & 27 & 3588 & 2723 & 27 & 15046 \\
\hline 29 & 41 & MSM & 6720 & 188 & 11461 & - & - & - & 1808 & 137 & 11084 & - & - & - \\
\hline 31 & 44 & MSM & 7988 & 110 & 1390 & - & - & - & 908 & 110 & 1407 & - & - & - \\
\hline 32 & 42 & MSM & 182 & 74 & 1247 & - & - & - & 1808 & 29 & 2992 & 1808 & 836 & $\begin{array}{l}\text { New } \\
\text { ST6 }\end{array}$ \\
\hline 33 & 21 & Women $^{a}$ & - & - & - & $\begin{array}{l}99 \%- \\
6405\end{array}$ & 74 & $\begin{array}{l}\text { New } \\
\text { ST7 }\end{array}$ & 6720 & 188 & 11461 & - & - & - \\
\hline 37 & 25 & MSM & 1808 & 188 & $\begin{array}{l}\text { New } \\
\text { ST8 }\end{array}$ & - & - & - & 182 & 74 & 1247 & - & - & - \\
\hline 39 & 20 & MSI & - & - & - & - & - & - & 3031 & 33 & 4995 & 4288 & 4 & 9382 \\
\hline 48 & 21 & MSM & - & - & - & - & - & - & 6720 & 188 & 11461 & 4288 & 188 & $\begin{array}{l}\text { New } \\
\text { ST9 }\end{array}$ \\
\hline 53 & 41 & MSM & 30 & 18 & 5441 & - & - & - & 3059 & 29 & 5049 & - & - & - \\
\hline 54 & 25 & MSM & - & - & - & - & - & - & 1808 & 29 & 2992 & 30 & 18 & 5441 \\
\hline 55 & 36 & Women $^{a}$ & - & - & - & 1808 & 29 & 2992 & 6720 & 188 & 11461 & - & - & - \\
\hline 58 & 49 & & 30 & 18 & 5441 & - & - & - & - & - & - & 1808 & 29 & 2992 \\
\hline 59 & 26 & MSI & - & - & - & - & - & - & 1808 & 29 & 2992 & 30 & 18 & 5441 \\
\hline 60 & 42 & MSM & 30 & 18 & 5441 & - & - & - & 1808 & 29 & 2992 & - & - & - \\
\hline 79 & 20 & MSM & - & - & - & - & - & - & 4199 & 29 & $\begin{array}{l}\text { New } \\
\text { ST14 }\end{array}$ & 4199 & 4 & $\begin{array}{l}\text { New } \\
\text { ST12 }\end{array}$ \\
\hline 80 & 19 & MSM & 6720 & 188 & 11461 & - & - & - & 30 & 29 & 298 & - & - & - \\
\hline
\end{tabular}

NG-MAST: Neisseria gonorrhoeae multi-antigen sequence typing; ST: sequence type.

${ }^{a}$ Women reporting anal sex or symptoms.

Sample material that was NG-negative or not sampled as part of routine diagnostics is indicated with a hyphen. 
The Figure presents the dissimilarity of concatenated sequences of por $B$ and $t b p B$ between STs. For the majority of the patients with distinct concurrent STs, a large (>15\%) dissimilarity was observed between the concatenated sequences. Patients 31 and 48 had only $1 \%$ dissimilarity between the concatenated sequences. In both patients, the tbpB allele was identical between the distinct ST but the porB allele showed $>1 \%$ dissimilarity, meaning the two ST did not belong to the same genogroup. When assigning ST to genogroups, we identified two genogroups that consisted of more than five samples: G2992 $(n=32)$ and G11084 $(n=6)$. Furthermore, we identified three samples belonging to G1407 (one ST1407 and two ST2212) of which only one (ST2212) was culture-positive and susceptible for ceftriaxone. 
Figure. Dendogram constructed by multiple alignment of concatenated porB and tbp $B$ sequences clustered with unweighted pair group method with arithmic mean (UPGMA) algorithm, the Netherlands, January 2012-May $2016(n=169)$

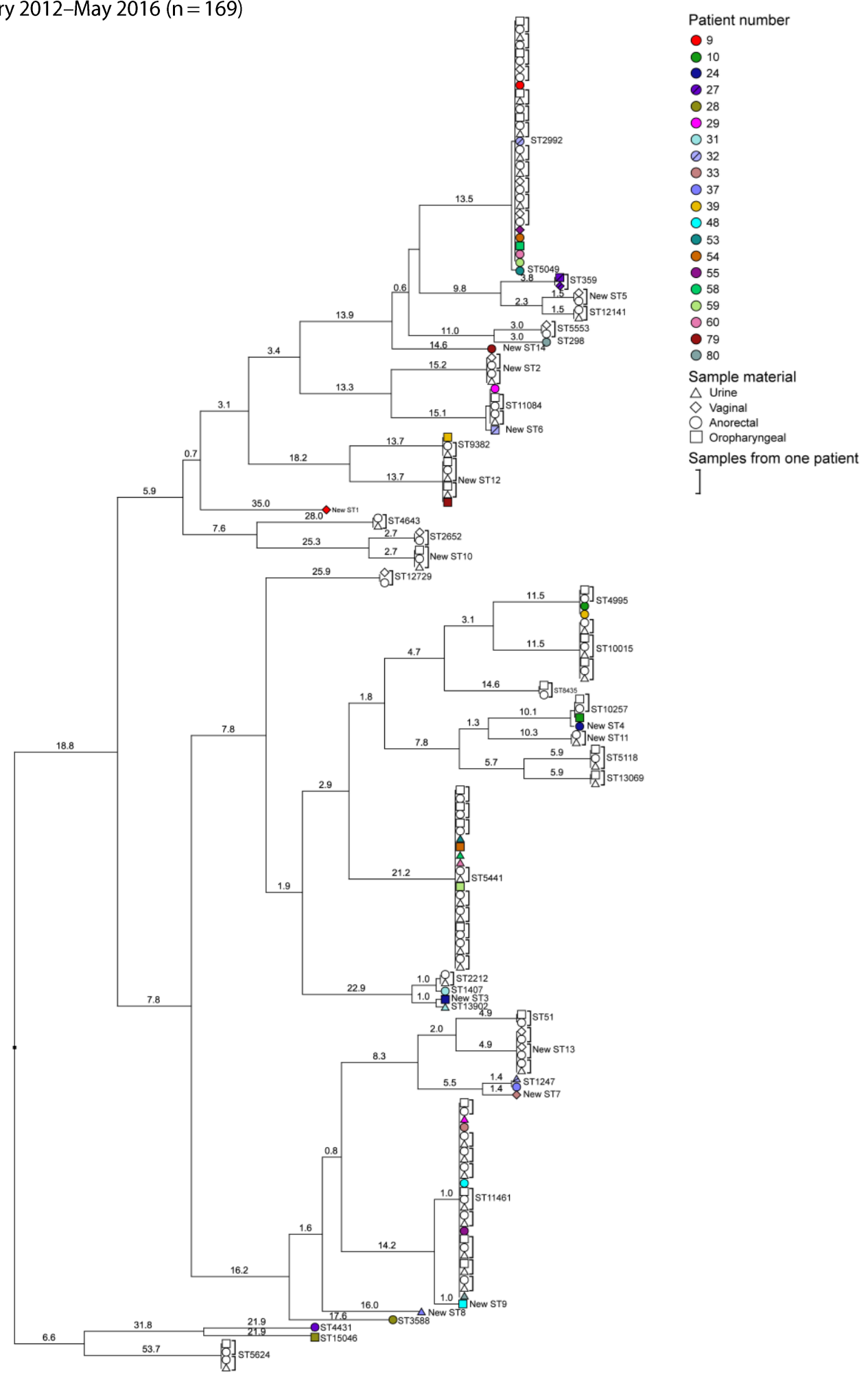

Sequence type (ST) of each sample is shown at the tips of the dendogram and samples of patients with distinct ST are coloured. The branch length depicts the percentage of dissimilarity. 


\section{Discussion}

In this study we show that the culture-free NG-MAST method can readily be used to genotype NG in clinical samples including extra-genital samples. In addition, the method is compatible with the online NG-MAST database. The culture-free NG-MAST method was technically validated by assessing the NG analytical specificity using nongonoccocal Neisseria species; it demonstrated good specificity for NG as no crossreactivity was observed. Furthermore, concordance with NG-MAST was demonstrated by comparing typing results of non-culturable clinical samples (Cobas 4800 sample material) with cultured isolates, which were taken less than 2 weeks apart. In this time frame, identical genotypes were expected based on the study by Martin et al. [7]. With culture-free NG-MAST, we genotyped $90 \%$ of the selected paired clinical samples with sufficient bacterial load ( $\mathrm{Cq}$ value $<35)$. Extrapolating this genotyping rate to all NG positive samples $(n=1,110)$ would result in successful typing of $56 \%(624 / 1,110)$ of all NG-positive samples. Among all samples sent in for culture, culture-free NG-MAST showed a higher typing rate of $94 \%$ (89/95) compared with the culture-dependent method with $62 \%$ (59/95). However, four of the 59 culture-positive samples were negative in culture-free NG-MAST. The clinical samples testing negative in culture-free NG-MAST could be caused by PCR-inhibitory substances in the clinical material. The majority of the culture-positive samples were collected from the genital site (44/59); that could be explained by the sampling strategy, but the low sensitivity of extra-genital NG culture could also have contributed [2]. This highlights the importance of culture-free genotyping as the current surveillance data would be biased towards genital samples. With our method, we were able to genotype 33 of the 36 culture-negative samples, which were mainly extra-genital samples (25/33).

Our results show that both sexes were frequently infected with distinct NG strains in a quarter of patients $(20 / 80)$ which is higher than most previous studies $[6,11,17,18]$. The studies of Whiley et al. (0/4) and Carannante et al. (1/8) assessed, respectively, only four and eight patients with paired samples, which could explain the lower proportion [6,11]. Pond et al. (3/71) developed a real-time PCR assay to predict ciprofloxacin resistance 
with the detection of a resistance-associated SNP [17]. This method uses a single position to identify distinct strains of NG, leading to a lower resolution than NG-MAST where two internal fragments (490 bp and $390 \mathrm{bp}$ ) of highly polymorphic genes are analysed. De Silva et al. (26/206) performed whole genome sequencing only on cultured strains and therefore may have missed distinct strains from samples that were NAATpositive but culture-negative [18]. A higher percentage of distinct strains in paired clinical samples was reported in a study by Kolader et al. (52/130) which applied por-opa restriction fragment length polymorphism typing [16]. The authors hypothesised that the observed high frequency could be the result of high-risk sexual behaviour or also of recombination in the opa genes.

High-risk behaviour and sex with multiple sex partners on the same occasion may explain the frequently observed distinct ST in our study as we included MSM and women reporting anal sex or symptoms attending our STI clinic, who are considered as risk groups $[1,16]$. Another possible reason could be DNA exchange with commensal Neisseria species or other NG strains $[11,16]$. Patients colonised with multiple NG strains could have different AMR profiles, potentially resulting in under-treatment which could allow dissemination of resistant strains $[6,17]$. However, the impact of multiple strain infections on treatment needs to be addressed in future research to answer questions of the effect on AMR development and dissemination of resistant strains.

The observed concurrent infections with distinct strains in our study would be overlooked in routine diagnostics as Dutch and European (European Centre for Disease Prevention and Control) NG resistance surveillance guidelines recommend culture of only one anatomical site $[2,20,21]$. Without typing data for concurrent NG infections, surveillance data are incomplete and potential transmission links or associations between ST and AMR can be missed. This potentially results in dissemination of unrecognised resistant types. Therefore, early detection and improved surveillance of ST that are linked to AMR could minimise sequelae and prevent dissemination of multidrug-resistant strains. 
We observed high variability in both alleles and ST in our study population, which could be due to sampling over a prolonged time period and from different risk groups (MSM and women reporting anal sex or symptoms). For example, ST2212, ST2992, ST5441 and ST5793 are more prevalent in MSM than heterosexual men or women [10,22,23]. In our study, these STs were mainly found in MSM, but eight samples with ST2992 and one with ST5793 were from women. Interestingly, we found three samples which belong to the genogroup G1407 (ST1407 ( $n=1$ ) and ST2212 ( $=2)$ ) linked to decreased susceptibility to the last first-line treatment with ceftriaxone [10,23]. Only one of the three could be cultured (ST2212) and was susceptible to ceftriaxone. Furthermore, ST359, ST2992, ST3588 and ST4995 are linked to azithromycin resistance which is the recommended dual-therapy treatment with ceftriaxone in case of a Chlamydia trachomatis co-infection [1,23-25]. In addition, this dual therapy is applied to slow down emerging resistance or where local resistance data are not available $[1,5,20]$. In the Netherlands, a single treatment with ceftriaxone is applied because no resistance has yet been found in the Netherlands [4]. However, a multidrug-resistant isolate was recently found in the United Kingdom that showed high-level resistance to both ceftriaxone and azithromycin, thereby highlighting the need for improved surveillance [26]. In our study population, we found a high prevalence of ST belonging to genogroup G2992 (19.5\%), which is in line with earlier data from the Netherlands (16.1\%), while genogroups G1407 (1.7\%) and G359 (1.1\%) were less frequent (respectively $7.7 \%$ and $6.3 \%$ in the study of Wind et al.) [23]. The genogroup G2992 is also frequently observed in most other countries in Europe [27] and G1407 prevalence is higher in most European countries than in the Netherlands.

Even though many NG-MAST ST are linked to resistance profiles in NG, this does not necessarily imply that the strain is phenotypically resistant $[7,10]$. Additional tests that can identify mutations leading to resistance, for example azithromycin resistance, could give more insight into those strains that cannot be cultured [28]. A limitation of this study is that we only included samples with a higher NG load (Cq value <35); therefore the typing rate of samples with a lower bacterial load is unknown. However, as culture success is also associated with bacterial load, culture-dependent methods are expected 
to perform worse than our culture-free method in samples with a low NG load (Cq value $235)$. This hypothesis is strengthened because only $10 \%(18 / 188)$ of the samples with a $\mathrm{Cq}$ value $\geq 35$ were culture-positive in routine diagnostics. A nested PCR approach might improve genotyping of samples with a low bacterial load as has been applied for medico legal purposes to allow typing from a piece of clothing [29].

\section{Conclusion}

The culture-free NG-MAST method can genotype NG from most non-culturable clinical samples, including extra-genital samples, as cross-reactivity with commensal Neisseria species was not observed. Compared with culture-dependent NG-MAST, culture-free NG-MAST has a higher typing rate and does not have high demands on sample conditions. Applying culture-free NG-MAST to clinical samples revealed frequent concurrent infections with distinct ST at separate anatomical sites in MSM and women reporting anal sex or symptoms. These distinct concurrent ST and extra-genital NG infections would be missed in the current European surveillance strategy possibly allowing dissemination of resistant NG strains. Including non-culturable and concurrent NG infections in surveillance informs actions to contain the dissemination of multidrugresistant NG strains. 


\section{References}

1. World Health Organization (WHO). Global action plan to control the spread and impact of antimicrobial resistance in Neisseria gonorrhoeae. Geneva: WHO; 2012. Available from: http://apps.who.int/iris/bitstream/handle/10665/44863/9789241503501_eng.pdf;jsessionid= DFA2F277BB3490186A525ABFEF62B3E5? sequence $=1$

2. Recommendations for the laboratory-based detection of Chlamydia trachomatis and Neisseria gonorrhoeae--2014. MMWR Recomm Rep. 2014;63(RR-02):1-19.

3. Goire N, Lahra MM, Chen M, Donovan B, Fairley CK, Guy R, et al. Molecular approaches to enhance surveillance of gonococcal antimicrobial resistance. Nat Rev Microbiol. 2014;12(3):223-

9. https://doi.org/10.1038/nrmicro3217 PMID: 24509781

4. Visser M, van Aar F, Op de Coul ELM, Slurink IAL, van Wees DA, Hoenderboom BM, et al. Sexually transmitted infections in the Netherlands in 2017. Bilthoven: National Institute for Public Health and Environment; 2018. Available from: https://www.rivm.nl/bibliotheek/rapporten/2018-0012.pdf

5. World Health Organization (WHO). WHO guidelines for the treatment of Neisseria gonorrhoeae. Geneva: WHO; 2016. Available

from: http://apps.who.int/iris/bitstream/handle/10665/246114/9789241549691eng.pdf?sequence $=1$

6. Carannante A, Ghisetti V, Dal Conte I, Gregori G, Stella ML, Vacca P, et al. Molecular characterization of Neisseria gonorrhoeae on non-cultured specimens from multiple anatomic sites. Ann Ist Super Sanita. 2017;53(3):213-7. PMID: 28956800

7. Martin IM, Ison CA, Aanensen DM, Fenton KA, Spratt BG. Rapid sequence-based identification of gonococcal transmission clusters in a large metropolitan area. J Infect Dis. 2004;189(8):1497505. https://doi.org/10.1086/383047 PMID: 15073688

8. Unemo M, Dillon JA. Review and international recommendation of methods for typing neisseria gonorrhoeae isolates and their implications for improved knowledge of gonococcal epidemiology, treatment, and biology. Clin Microbiol Rev. 2011;24(3):447-58. https://doi.org/10.1128/CMR.0004010 PMID: 21734242

9. Kwong JC, Gonçalves da Silva A, Dyet K, Williamson DA, Stinear TP, Howden BP, et al. NGMASTER:in silico multi-antigen sequence typing for Neisseria gonorrhoeae. Microb Genom. 2016;2(8):e000076. PMID: 28348871

10. Chisholm SA, Unemo M, Quaye N, Johansson E, Cole MJ, Ison CA, et al. Molecular epidemiological typing within the European Gonococcal Antimicrobial Resistance Surveillance Programme reveals predominance of a multidrug-resistant clone. Euro Surveill. 2013;18(3):20358. PMID: 23351652

11. Whiley DM, Goire N, Ray ES, Limnios A, Lambert SB, Nissen MD, et al. Neisseria gonorrhoeae multiantigen sequence typing using non-cultured clinical specimens. Sex Transm Infect. 2010;86(1):515. https://doi.org/10.1136/sti.2009.037689 PMID: 19843535

12. Dukers-Muijrers NH, Schachter J, van Liere GA, Wolffs PF, Hoebe CJ. What is needed to guide testing for anorectal and pharyngeal Chlamydia trachomatis and Neisseria gonorrhoeae in women and men? Evidence and opinion. BMC Infect Dis. 2015;15(1):533. https://doi.org/10.1186/s12879-0151280-6 PMID: 26576538

13. Chan PA, Janvier M, Alexander NE, Kojic EM, Chapin K. Recommendations for the diagnosis of Neisseria gonorrhoeae and Chlamydia trachomatis, including extra-genital sites. Med Health $\mathrm{R}$ I. 2012;95(8):252-4. PMID: 22970467

14. Unemo M, Shafer WM. Antimicrobial resistance in Neisseria gonorrhoeae in the 21st century: past, evolution, and future. Clin Microbiol Rev. 2014;27(3):587-613. https://doi.org/10.1128/CMR.0001014 PMID: 24982323

15. Bissessor M, Tabrizi SN, Fairley CK, Danielewski J, Whitton B, Bird S, et al. Differing Neisseria gonorrhoeae bacterial loads in the pharynx and rectum in men who have sex with men: implications for gonococcal detection, transmission, and control. J Clin Microbiol. 2011;49(12):43046. https://doi.org/10.1128/JCM.05341-11 PMID: 21956992

16. Kolader ME, Dukers NH, van der Bij AK, Dierdorp M, Fennema JS, Coutinho RA, et al. Molecular epidemiology of Neisseria gonorrhoeae in Amsterdam, The Netherlands, shows distinct heterosexual and homosexual networks. J Clin Microbiol. 2006;44(8):2689-

97. https://doi.org/10.1128/JCM.02311-05 PMID: 16891479 
17. Pond MJ, Hall CL, Miari VF, Cole M, Laing KG, Jagatia H, et al. Accurate detection of Neisseria gonorrhoeae ciprofloxacin susceptibility directly from genital and extragenital clinical samples: towards genotype-guided antimicrobial therapy. J Antimicrob Chemother. 2016;71(4):897902. https://doi.org/10.1093/jac/dkv432 PMID: 26817487

18. De Silva D, Peters J, Cole K, Cole MJ, Cresswell F, Dean G, et al. Whole-genome sequencing to determine transmission of Neisseria gonorrhoeae: an observational study. Lancet Infect Dis. 2016;16(11):1295-303. https://doi.org/10.1016/S1473-3099(16)30157-8 PMID: 27427203

19. Unemo M, Golparian D, Sánchez-Busó L, Grad Y, Jacobsson S, Ohnishi M, et al. The novel 2016 WHO Neisseria gonorrhoeae reference strains for global quality assurance of laboratory investigations: phenotypic, genetic and reference genome characterization. J Antimicrob Chemother. 2016;71(11):3096-108. https://doi.org/10.1093/jac/dkw288 PMID: 27432602

20. Workowski KA, Bolan GACenters for Disease Control and Prevention. Sexually transmitted diseases treatment guidelines, 2015. MMWR Recomm Rep. 2015;64(RR-03):1-137. PMID: 26042815

21. European Centre for Disease Prevention and Control (ECDC). Gonococcal antimicrobial susceptibility surveillance in Europe 2015. Stockholm: ECDC; 2017. Available from: https://ecdc.europa.eu/sites/portal/files/documents/gonococcal-antimicrobial-susceptibilitysurveillance-Europe-2015.pdf

22. Cheng CW, Li LH, Su CY, Li SY, Yen MY. Changes in the six most common sequence types of Neisseria gonorrhoeae, including ST4378, identified by surveillance of antimicrobial resistance in northern Taiwan from 2006 to 2013. J Microbiol Immunol Infect. 2016;49(5):70816. https://doi.org/10.1016/j.jmii.2014.08.016 PMID: 25442864

23. Wind CM, Bruisten SM, Schim van der Loeff MF, Dierdorp M, de Vries HJC, van Dam AP. A CaseControl Study of Molecular Epidemiology in Relation to Azithromycin Resistance in Neisseria gonorrhoeae Isolates Collected in Amsterdam, the Netherlands, between 2008 and 2015. Antimicrob Agents Chemother. 2017;61(6):e02374-16. https://doi.org/10.1128/AAC.0237416 PMID: 28373191

24. Shigemura K, Osawa K, Miura M, Tanaka K, Arakawa S, Shirakawa T, et al. Azithromycin resistance and its mechanism in Neisseria gonorrhoeae strains in Hyogo, Japan. Antimicrob Agents Chemother. 2015;59(5):2695-9. https://doi.org/10.1128/AAC.04320-14 PMID: 25712352

25. Brunner A, Nemes-Nikodem E, Jeney C, Szabo D, Marschalko M, Karpati S, et al. Emerging azithromycin-resistance among the Neisseria gonorrhoeae strains isolated in Hungary. Ann Clin Microbiol Antimicrob. 2016;15(1):53. https://doi.org/10.1186/s12941-016-0166-9 PMID: 27646968

26. Eyre DW, Sanderson ND, Lord E, Regisford-Reimmer N, Chau K, Barker L, et al. Gonorrhoea treatment failure caused by a Neisseria gonorrhoeae strain with combined ceftriaxone and high-level azithromycin resistance, England, February 2018. Euro Surveill. 2018;23(27):1800323. https://doi.org/10.2807/1560-7917.ES.2018.23.27.1800323 PMID: 29991383

27. European Centre for Disease Prevention and Control (ECDC). Molecular typing of Neisseria gonorrhoeae - a study of 2013 isolates. Stockholm: ECDC; 2018. Available from: https://ecdc.europa.eu/sites/portal/files/documents/Molecular-typing-N-gonorrhoeaeweb.pdf

28. Trembizki E, Buckley C, Donovan B, Chen M, Guy R, Kaldor J, et al. Direct real-time PCR-based detection of Neisseria gonorrhoeae 23S rRNA mutations associated with azithromycin resistance. J Antimicrob Chemother. 2015;70(12):3244-9. PMID: 26338048

29. Martin IM, Foreman E, Hall V, Nesbitt A, Forster G, Ison CA. Non-cultural detection and molecular genotyping of Neisseria gonorrhoeae from a piece of clothing. J Med Microbiol. 2007;56(Pt 4):48790. https://doi.org/10.1099/jmm.0.46956-0 PMID: 17374888 


\section{Supplemental files}
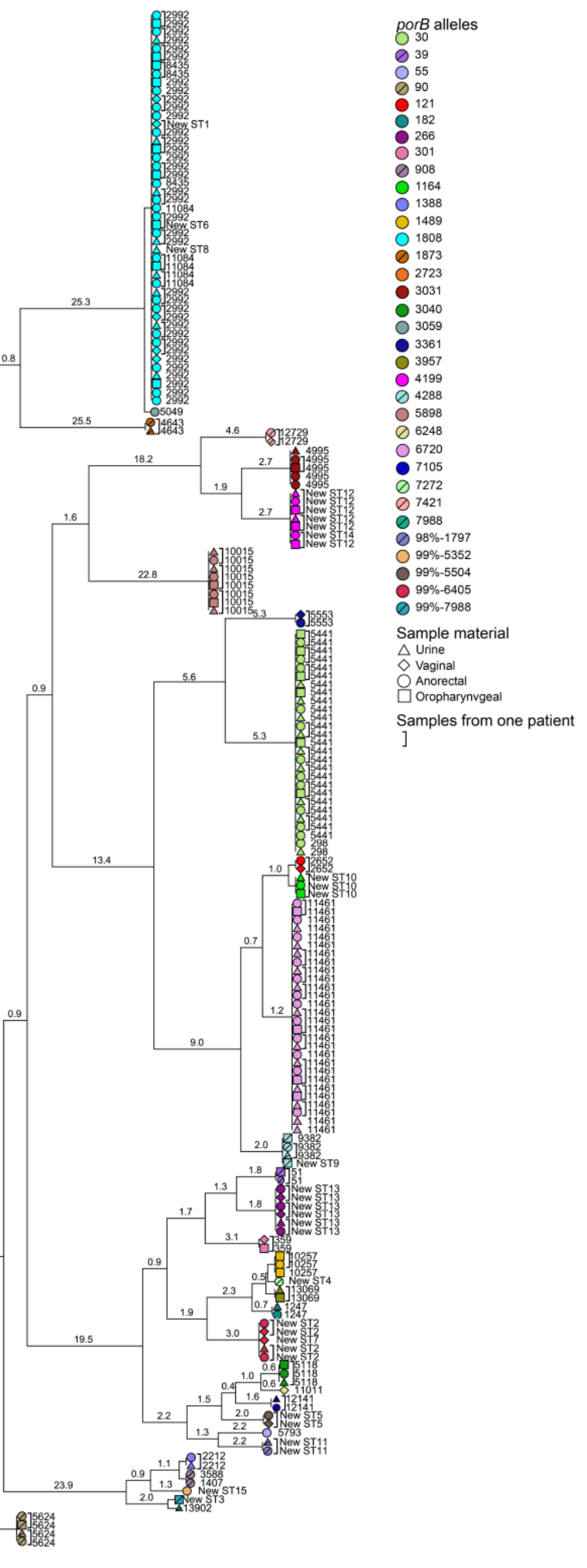

$$
\text { ] }
$$




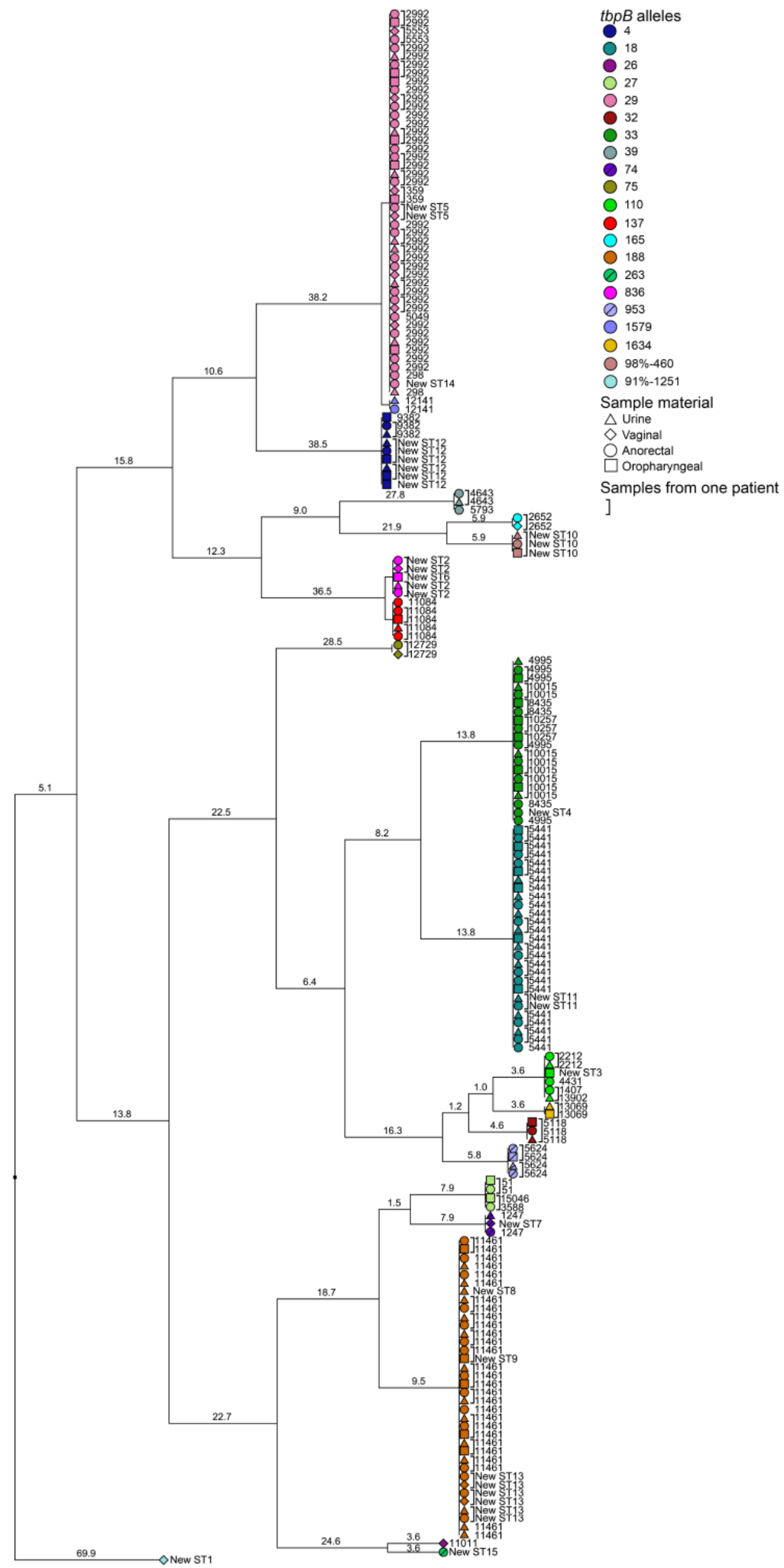

Supplemental figure 4 Dendogram constructed by multiple alignment of $\operatorname{tbp} B$ sequences $(n=185)$ clustered with Unweighted Pair Group Method with Arithmic mean (UPGMA) algorithm. Sequence type of each sample is shown at the tips of the dendogram and samples are coloured according to the respective $t b p B$ allele. The branch length depicts the percentage of dissimilarity. 


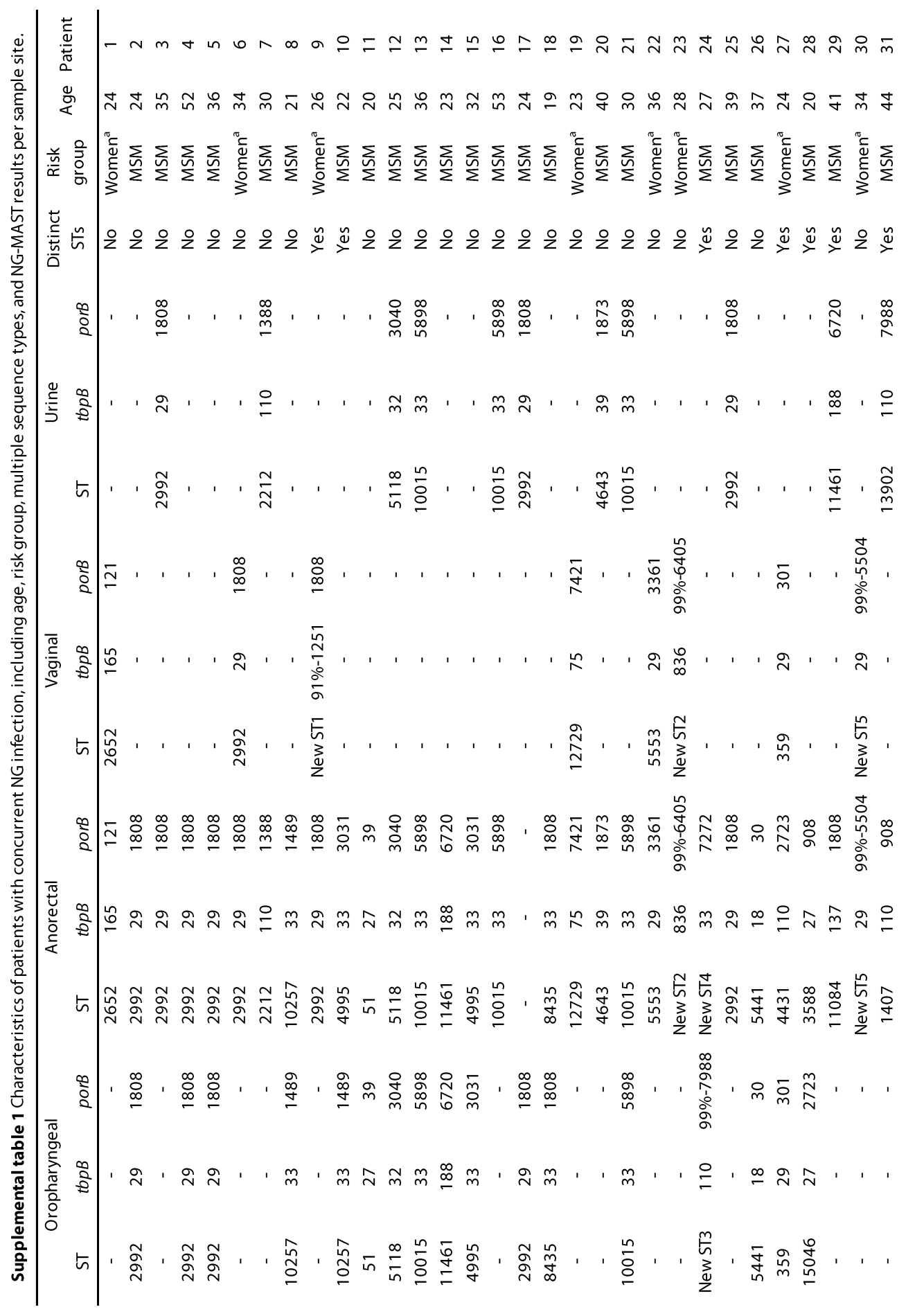




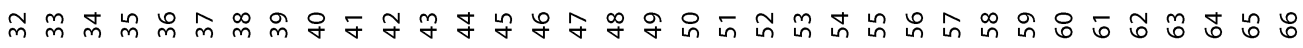

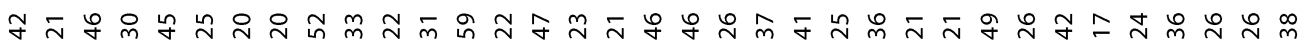

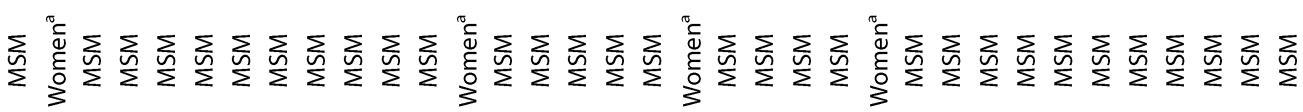
๗

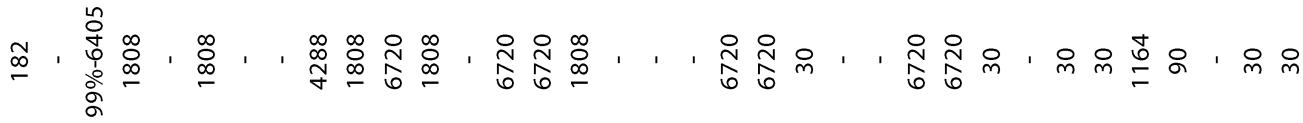

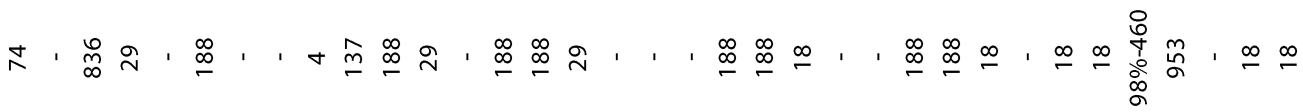

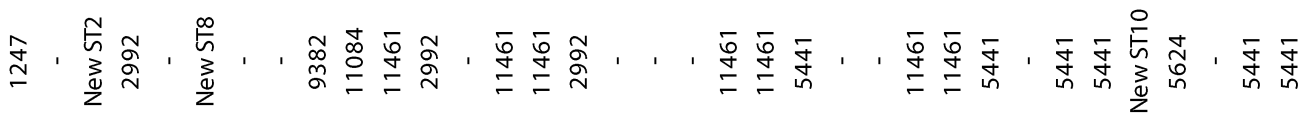

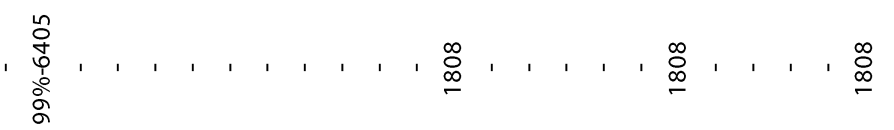

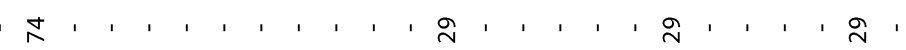

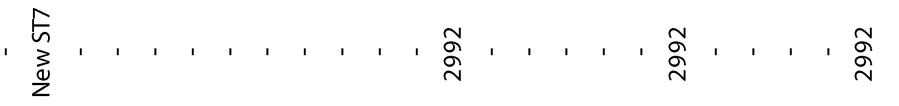

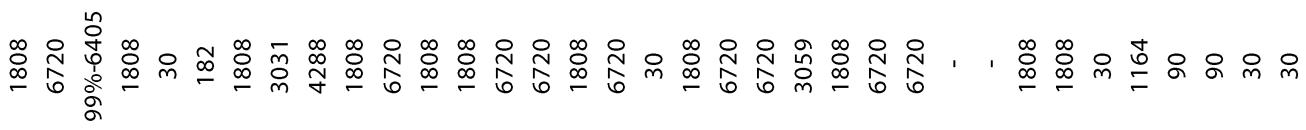

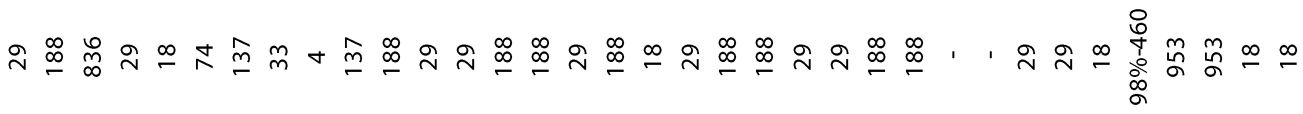

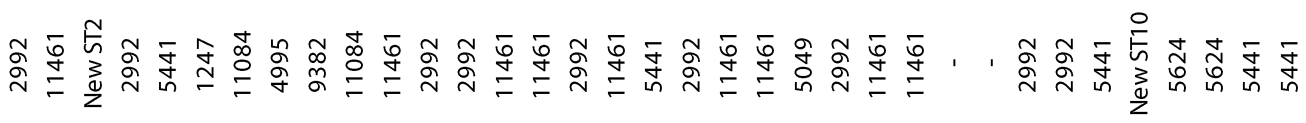

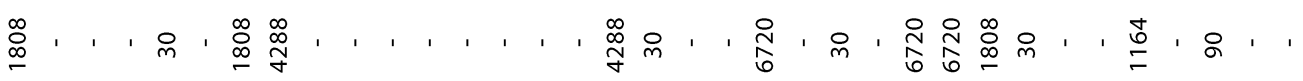

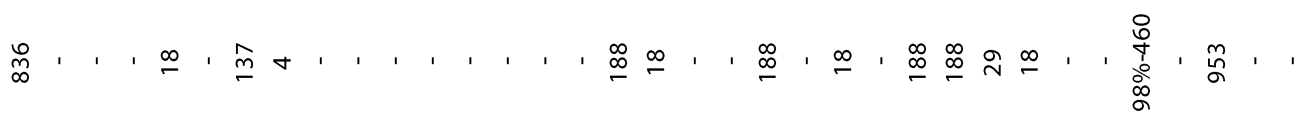

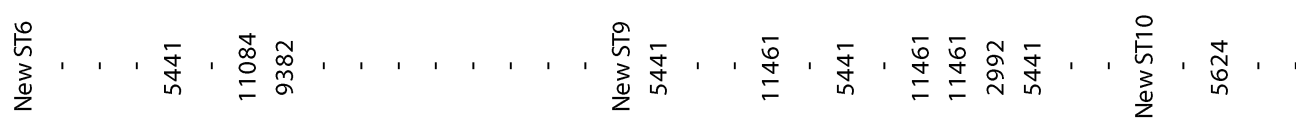


ง

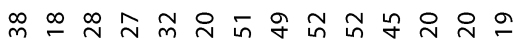

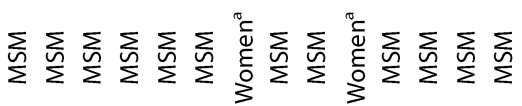

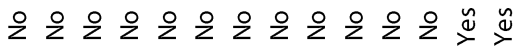

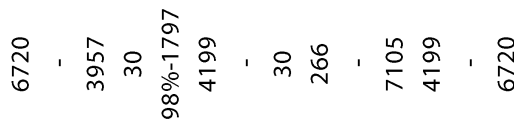

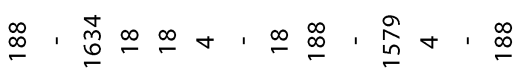

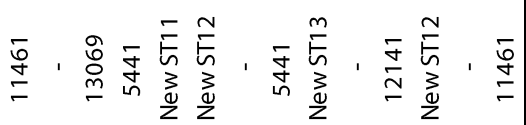
, , , , , , 导, , 导, , , , , ' , , , , $\stackrel{\infty}{\infty}, \stackrel{\infty}{\infty}_{\infty}^{\infty}, \quad, \quad$, $, \ldots, \frac{\sum^{m}}{\frac{m}{E}}, \frac{\sum^{m}}{\frac{m}{E}}, \ldots$, 帘o

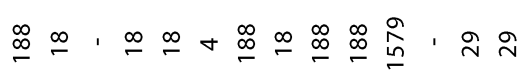

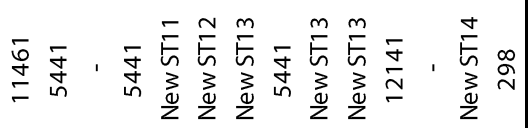

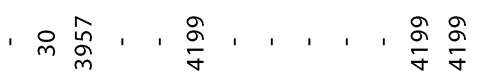

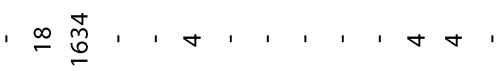

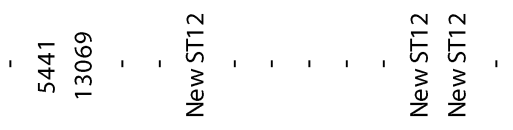





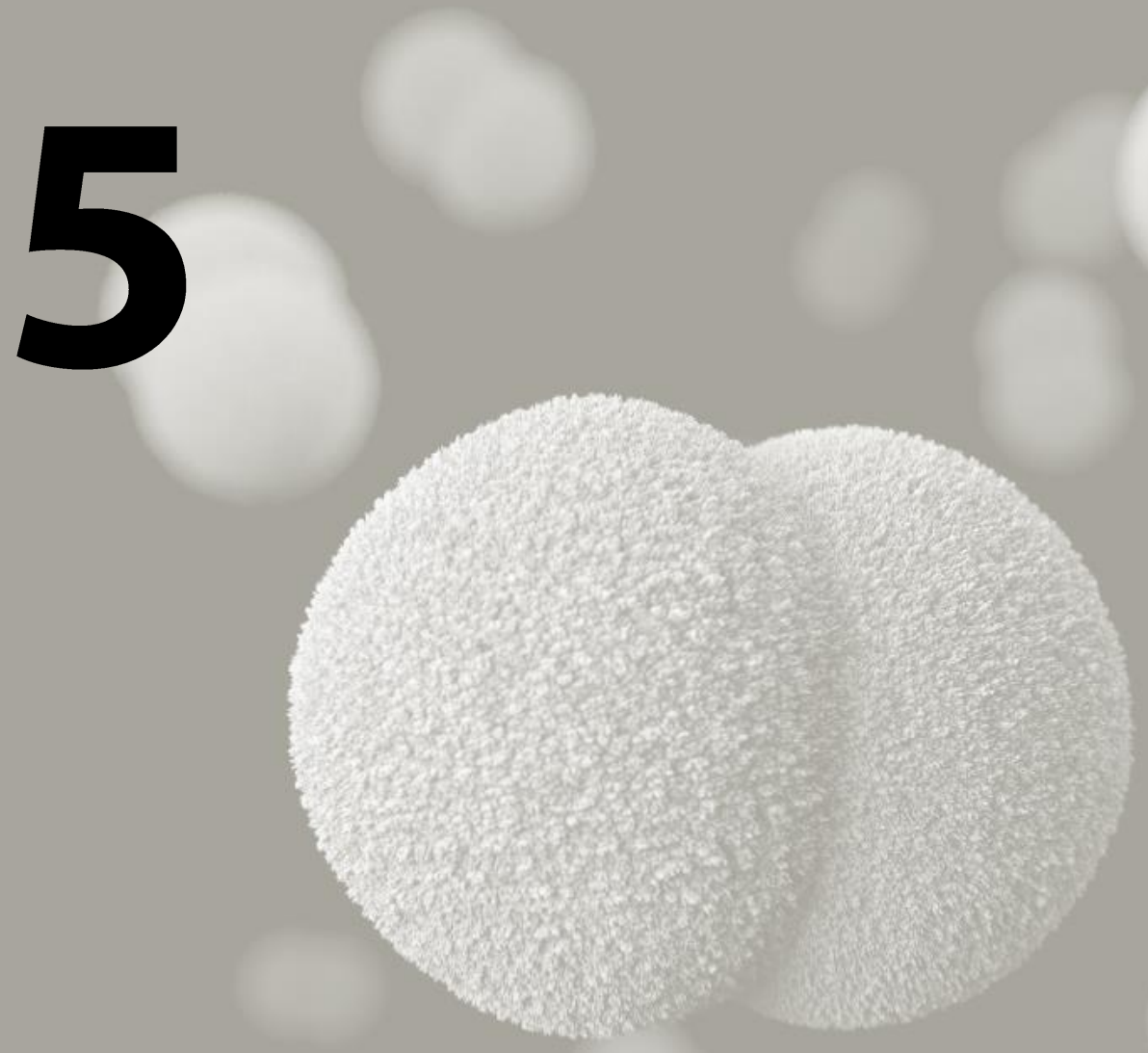




\section{Metagenomic analysis shows distinct Neisseria gonorrhoeae strains at separate anatomical sites occur more commonly than mixed strain infections: implications for surveillance}

Brian M.J.W. van der Veer, Petra F.G. Wolffs, Christian J.P.A. Hoebe,

Nicole H.T.M. Duker-Muijrers, Lieke B. van Alphen 


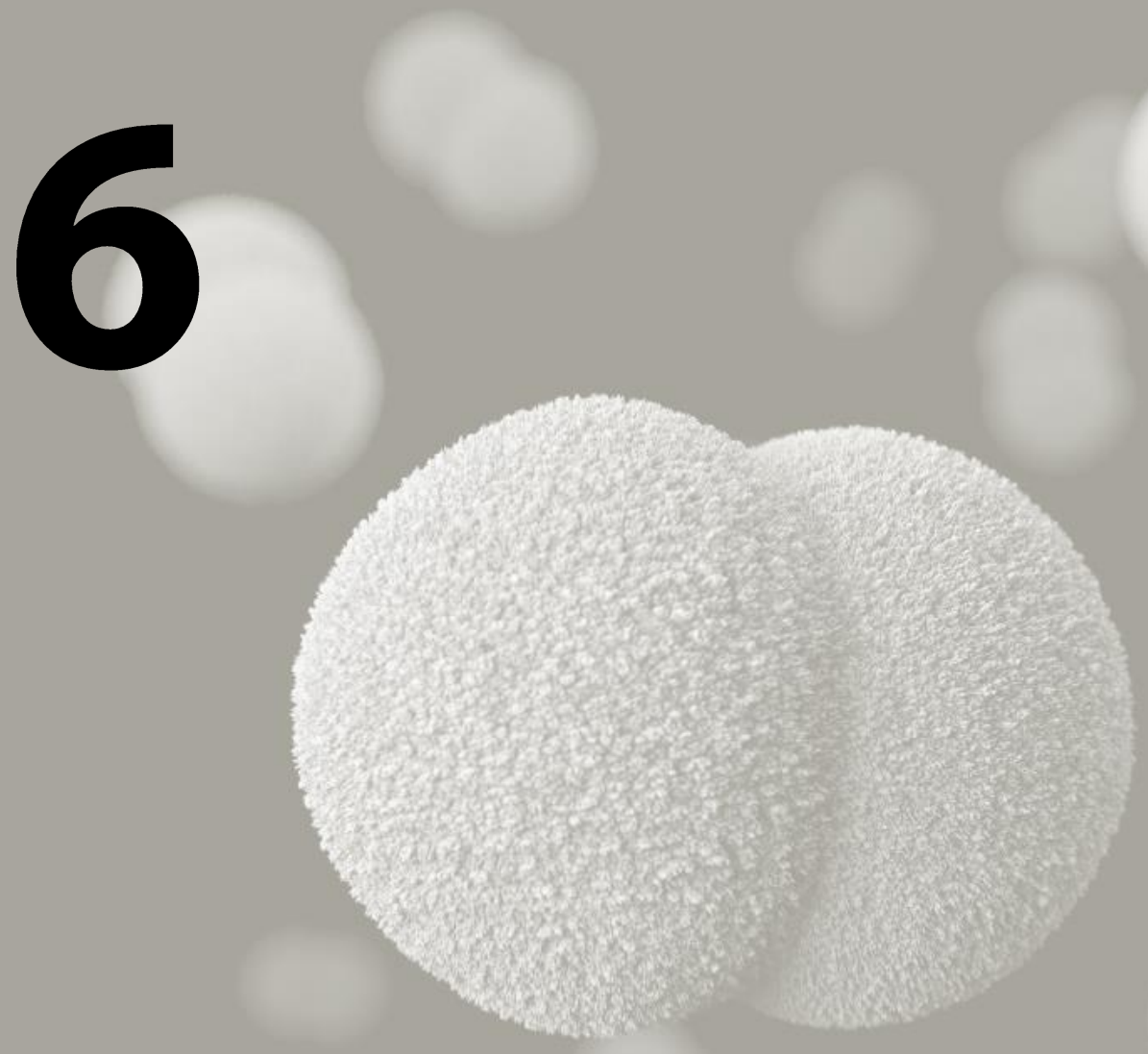




\section{Culture-free Genotyping Improves Surveillance of Neisseria gonorrhoeae, Especially in Oropharyngeal Samples, the Netherlands, 2017 to 2018}

Michiel H.C. Slaats, Brian M.J.W. van der Veer, Lieke B. van Alphen, Christian J.P.A. Hoebe, Nicole H.T.M. Dukers-Muijrers, Petra F.G. Wolffs 


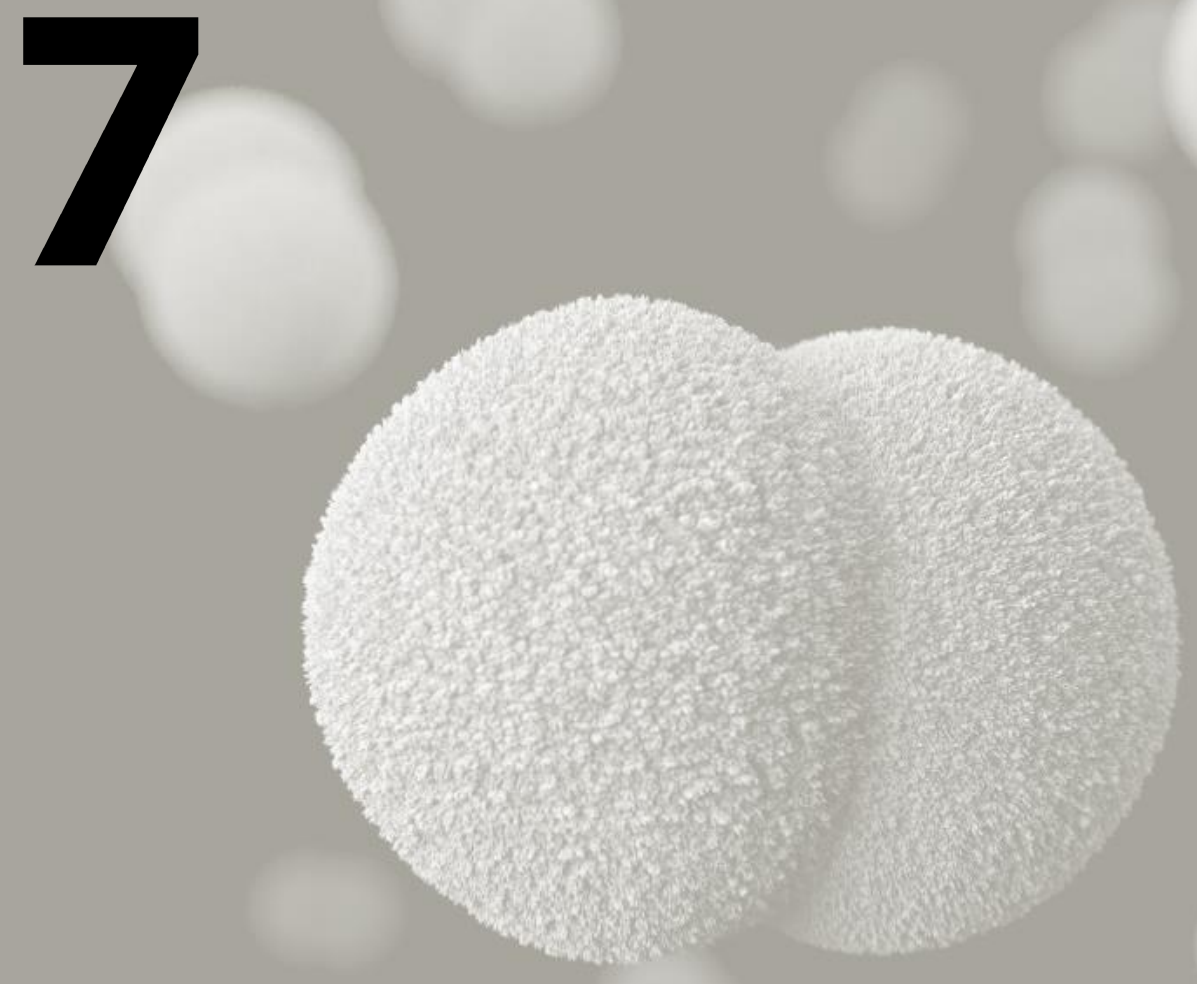




\section{Potential Neisseria gonorrhoeae outbreak in young heterosexuals $(<25)$; culture-independent genotyping revealed three clusters, the Netherlands, October 2017 to March 2019}

Brian M.J.W. van der Veer, Petra F.G. Wolffs, Christian J.P.A. Hoebe, Juliën N.A.P. Wijers, Gènevieve A.F.S. van Liere, Marita I.L.S. Werner, Amanja Verhaegh, Nicole H.T.M. Duker-Muijrers, Lieke B. van Alphen 


\section{Abstract}

In the last quarter of 2017 , seven young $(<25)$ women were diagnosed with genital Neisseria gonorrhoeae (NG) at the STI clinic in South-Limburg (the Netherlands), compared to only five young women previously in the year 2017. As triage and testing had not been changed this could indicate a potential outbreak. Further outbreak investigation demonstrated that 81 cases of young heterosexuals were observed between October 2017 to March 2019 (4.5 per month) compared to 30 young heterosexuals between January 2016 to September 2017 (1.4 per month) which was considered as baseline. Culture-independent genotyping of NG was performed to assess the genetic relatedness as only 21 outbreak cases were culture confirmed. This analysis revealed three independent outbreak clusters G2 ( $n=18), G 13113(n=11)$, and G NewST $(n=24)$. None of the clusters were geographically linked. Number of sex partners reported by men and Chlamydia trachomatis co-infection were associated with cluster G2 and G NewST respectively. No indication of bridging from MSM networks was found as possible introduction of NG in the young heterosexual population. Targeted interventions were difficult as information on sex partners was limited. Therefore, prospective culture-independent typing and an additional questionnaire were implemented to inform further control measures. 


\section{Background}

Neisseria gonorrhoeae (NG) is a common bacterial sexually transmitted infection (STI) that can infect genital, anorectal, oropharyngeal, and ocular mucosa [1-3]. Many NG infections, especially anorectal and oropharyngeal infections, are asymptomatic which could lead to unnoticed transmission [4, 5]. Therefore, it is a major public health challenge as the incidence rate remains high despite improved diagnostics $[1,6]$. NG is commonly transmitted during unprotected sexual activity but can also be transmitted from mother to child during birth $[2,3,7]$. Another potential route of transmission is through contact with saliva $[8,9]$. Key populations with a higher risk to acquire NG such as men who have sex with men (MSM) have been identified [4]. This is reflected in a higher positivity rate among MSM (11.0\%) compared to women (1.6\%) and heterosexual men (1.8\%) in the Netherlands [10].

Molecular epidemiology can have an important contribution in the elucidation of sexual networks, in addition to collected epidemiological data [11]. NG multi-antigen sequence typing (NG-MAST) and whole-genome sequencing have been applied to elucidate transmission networks and outbreaks of NG [12-19]. These studies revealed sustained transmission of some lineages in both MSM and heterosexuals resulting in strain clusters $[12,13,15-17,19]$. Also, bridging between MSM and heterosexual sexual networks has been demonstrated previously [16]. Furthermore, some studies reveal outbreaks of NG in distinct sexual networks or geographical areas $[12,13,18]$.

Strain clusters of NG in heterosexuals have been described previously in the UK $[12,13]$. Significant differences in behaviour and demographics of individuals between strain clusters were found [12]. Therefore, the authors hypothesize that strain clusters could represent localised transmission by distinct sexual networks. Some of the strain clusters identified by Risley et al. were associated with a geographical area [13]. The strain clusters that were not associated with a geographical area had a longer mean duration in the population and therefore might represent endemic strains. 


\section{Outbreak detection}

We noticed a potential outbreak of NG in the young heterosexual population visiting our STI clinic after we recognized an increasing number of young women with genital NG compared with an increasing number of young heterosexual men, while triage and the number of tests did not change since 2015 [20]. In the last quarter of 2017 seven young $(<25)$ women were diagnosed with genital NG visiting our STI clinic while in the previous three quarters of 2017, only five young women were diagnosed with genital NG. Here, we report the main findings of molecular outbreak investigation and demonstrate the added value of culture-independent genotyping and discuss challenges associated with NG outbreak detection and management.

\section{Methods}

\section{Case definition and data collection}

Confirmed cases related to this outbreak were defined as young $(<25)$ heterosexuals (women and men) living in the region South-Limburg in the Netherlands with a genital NG infection from October 2017 till March 2019. Extra-genital samples were not included as heterosexuals are not routinely tested at those anatomical sites as recommended by the Dutch STI guidelines [20]. NG and Chlamydia trachomatis (CT) infections were diagnosed in routine diagnostics using the Cobas $^{\oplus} 4800$ CT/NG test using a selfcollected vaginal swab (women) or first-void urine sample (men). As part of routine diagnostic procedures, a standardised questionnaire regarding the medical and sexual history of the last six months was collected by trained nurses. Data on sexual risk group, symptoms, number of sex partners, educational level, residence (four-digit postcode), and ethnicity were used in this study. The following were considered as urogenital symptoms: genital discharge, bleeding, itching, swelling, pain, burning sensation and more frequent urination. Educational level was defined as low/medium (no education, primary school or vocational) and high (higher education (secondary school) or university). Ethnicity was categorised as western and non-western background according to the Dutch consultation manual [20]. All missing data were handled as unknown and were not included in the analyses. 


\section{Outbreak investigation}

There were 81 confirmed cases ( 57 women and 24 men) in the timeframe of October 2017 - March 2019 compared to 30 young heterosexuals (17 women and 13 men) with genital NG from January 2016 to September 2017 which was considered as baseline. This resulted in a threefold higher number of genital NG-positive young heterosexuals per month during the outbreak (4.5 per month) compared to baseline (1.4 per month). Three outbreak cases were excluded due to lack of residual material for genotyping, leaving 78 cases (56 women and 22 men), of which 21 (26.9\%) were culture confirmed, available for analysis. Residual samples of routine diagnostics were genotyped using culture-free NGMAST to assess the genetic relatedness of the NG strains [21]. For comparison, residual NG-positive genital material of young heterosexuals at baseline was genotyped to determine the occurrence of clustering in this population. In addition, residual NGpositive genital material of MSM, including bisexual men, in 2018 ( $n=47)$ were genotyped to check for potential introduction of NG in the heterosexual population via bridging.

\section{Culture-independent genotyping}

Culture-free NG-MAST was performed as previously described, with minor modifications [21]. PCR protocols were modified as follows to improve sensitivity. The porB PCR protocol: $5 \mathrm{~min}$ at $95^{\circ} \mathrm{C}, 12$ cycles of $30 \mathrm{~s}$ at $95^{\circ} \mathrm{C}, 3 \mathrm{~min}$ at $68^{\circ} \mathrm{C}$ (decreasing by $0.5^{\circ} \mathrm{C}$ per cycle), 30 cycles of $30 \mathrm{~s}$ at $95^{\circ} \mathrm{C}, 1 \mathrm{~min}$ at $58^{\circ} \mathrm{C}, 2.5 \mathrm{~min}$ at $68^{\circ} \mathrm{C}$, and a final extension of 10 $\min$ at $68^{\circ} \mathrm{C}$. The tbpB PCR protocol: $5 \mathrm{~min}$ at $95^{\circ} \mathrm{C}, 12$ cycles of $30 \mathrm{~s}$ at $95^{\circ} \mathrm{C}, 3 \mathrm{~min}$ at $72^{\circ} \mathrm{C}$ (decreasing by $0.5^{\circ} \mathrm{C}$ per cycle), 30 cycles of $30 \mathrm{~s}$ at $95^{\circ} \mathrm{C}, 1 \mathrm{~min}$ at $65^{\circ} \mathrm{C}, 2.5 \mathrm{~min}$ at $68^{\circ} \mathrm{C}$, and a final extension of $10 \mathrm{~min}$ at $68^{\circ} \mathrm{C}$.

\section{Data analysis}

The Sanger sequence data analysis was performed as described previously [21]. Genogroups were assigned if $\geq 10$ cases had a shared identical allele and the other $\geq 99 \%$ similarity and were considered as a cluster [22]. Genogroups were used to ensure robust statistical analysis as proposed by Chisholm et al. and because some known sexual contacts do not have identical NG-MAST sequence types [22, 23]. 
Geospatial coordinates (residence) of outbreak cases were extracted using the R-package "nlgeocoder" version 0.1.3. We visualized residence areas of patients, based on their 4 digit postcode, per outbreak cluster in a geographical map using Qgis 2.18.28 [24]. In addition, the coordinates were used to test whether outbreak clusters, based on NG-MAST, were associated with a geographical area using SatScan (version 9.6) using the multinomial model [25].

Differences in epidemiological characteristics (sex, CT co-infection, educational level, notified for an STI, symptoms, and ethnicity) between strain clusters were pair-wise compared with the fisher-exact test. Student's T-test was used to test associations with number of sex-partners between strain clusters and between women and men within a strain cluster. These analyses were performed using $R$ statistical software version 3.6.2 ( $R$ Foundation for Statistical Computing, Vienna, Austria).

\section{Ethical statement}

The data in this study were obtained from medical records from routine care of our STI clinic in a de-identified manner. None of the researchers had access to patient identifying information. The STI clinic uses the 'no objection' informed consent and persons who refused use of data or samples are not included in this study. The study protocol was exempt from formal medical-ethical approval under prevailing laws in the Netherlands as it concerns only an retrospective observational study using anonymous data only (as stated by the National Central Committee for Human Studies: www.ccmo.nl and in the conduct of good behaviour in research www.federa.org).

\section{Results}

\section{Descriptive epidemiology}

A total of 81 cases were identified (57 women and 24 men) in the timeframe of October 2017 - March 2019 (figure 1A). As the increase in genital NG cases in young women was notified in the last quartile of 2017, all young heterosexuals with genital NG from January 2016 till September 2017 were considered baseline $(n=30,17$ women and 13 men)(figure 1A). Of the potential outbreak cases three were excluded due to lack of residual material for genotyping but none of the baseline. 
A

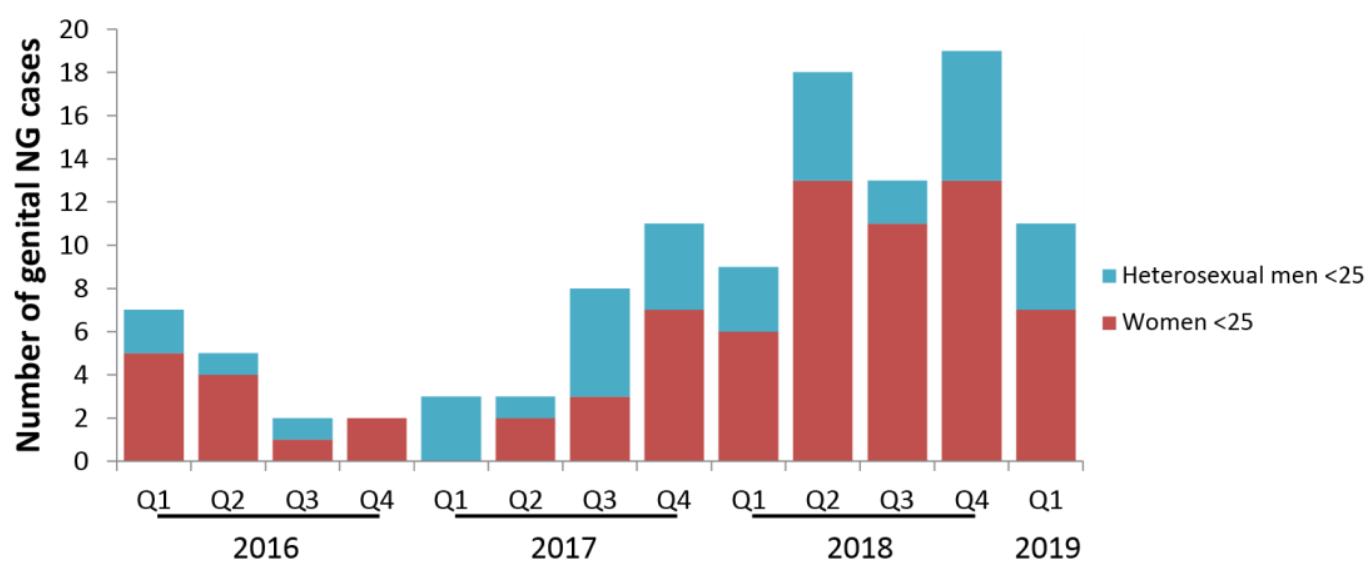

B

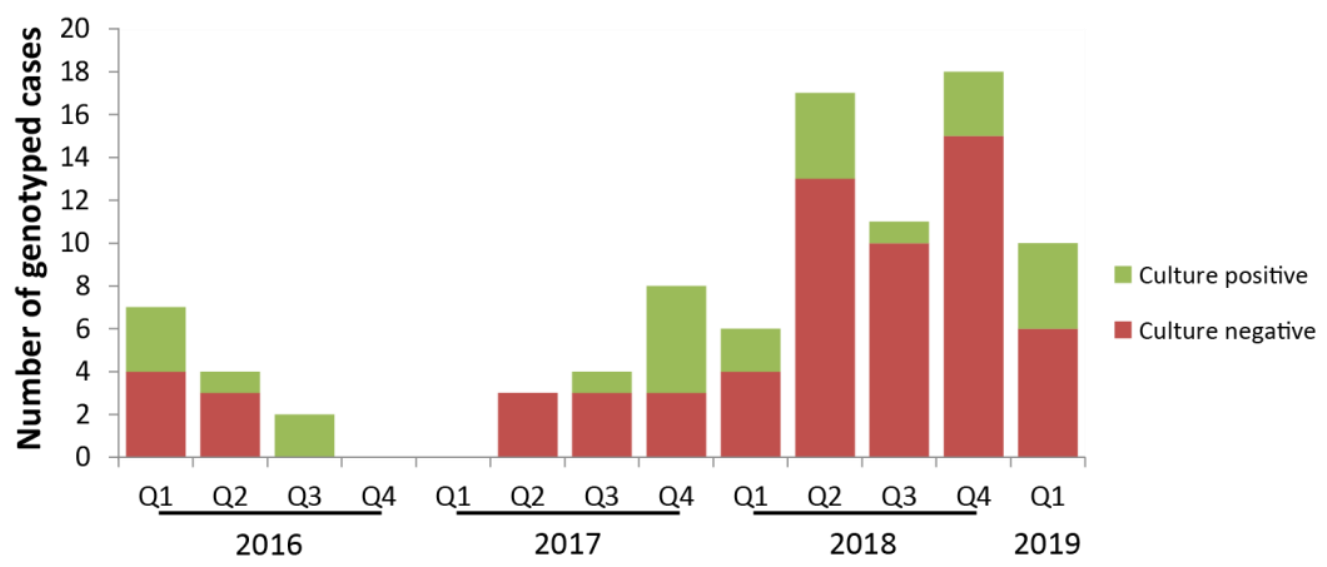

Figure $1 \mathrm{~A}$. Epidemiological curve of genital NG cases in young heterosexuals in South-Limburg the Netherlands. B. Epidemiological curve of genotyped samples of young heterosexuals in SouthLimburg the Netherlands. Bars are coloured according to the availability of cultured isolate. 


\section{Three independent outbreak clusters}

In total, 128/155 (82.6\%) of genital NG-positive samples were successfully genotyped with culture-free NG-MAST, 20/30 (66.7\%) baseline, 70/78 (89.7\%) of confirmed outbreak cases, and 38/47 (80.9\%) of MSM. Of the genotyped samples only 57/128 (44.5\%) were culture positive. Of the 27 samples that failed, 5 showed potential mixed strain infections (4 culture-positive) and 8 had a low bacterial load $\left(<2.8 \log _{10} \mathrm{CFU} / \mathrm{mL}\right.$, no culturepositives). The remaining 14 samples ( 2 culture-positive) could have failed due to PCR inhibition. The majority of the genotyped samples at baseline and during outbreak were not culture confirmed and thus could only be typed by culture independent methods (figure 1B). Figure 2 presents the dissimilarity of concatenated por $B$ and tbp $B$ sequences revealing three independent outbreak clusters. Namely G2 $(n=18), G 13113(n=11)$, and G NewST $(n=24)$. Of these outbreak clusters only $5 / 18,2 / 11$, and $6 / 24$ of cases had a cultured isolate and therefore would not have met the outbreak cluster criteria if a culture-dependent method was used. All genogroups, G2 $(n=1), G 13113(n=1)$ and G NewST $(n=3)$, were already present in the young heterosexual population before the increase of genital NG, in January 2016 (G13113) and between May and September 2017 (G2 and G NewST) respectively (figure 3). No genogroups were seen in young heterosexual cases pre-outbreak and MSM cases (figure 2). 


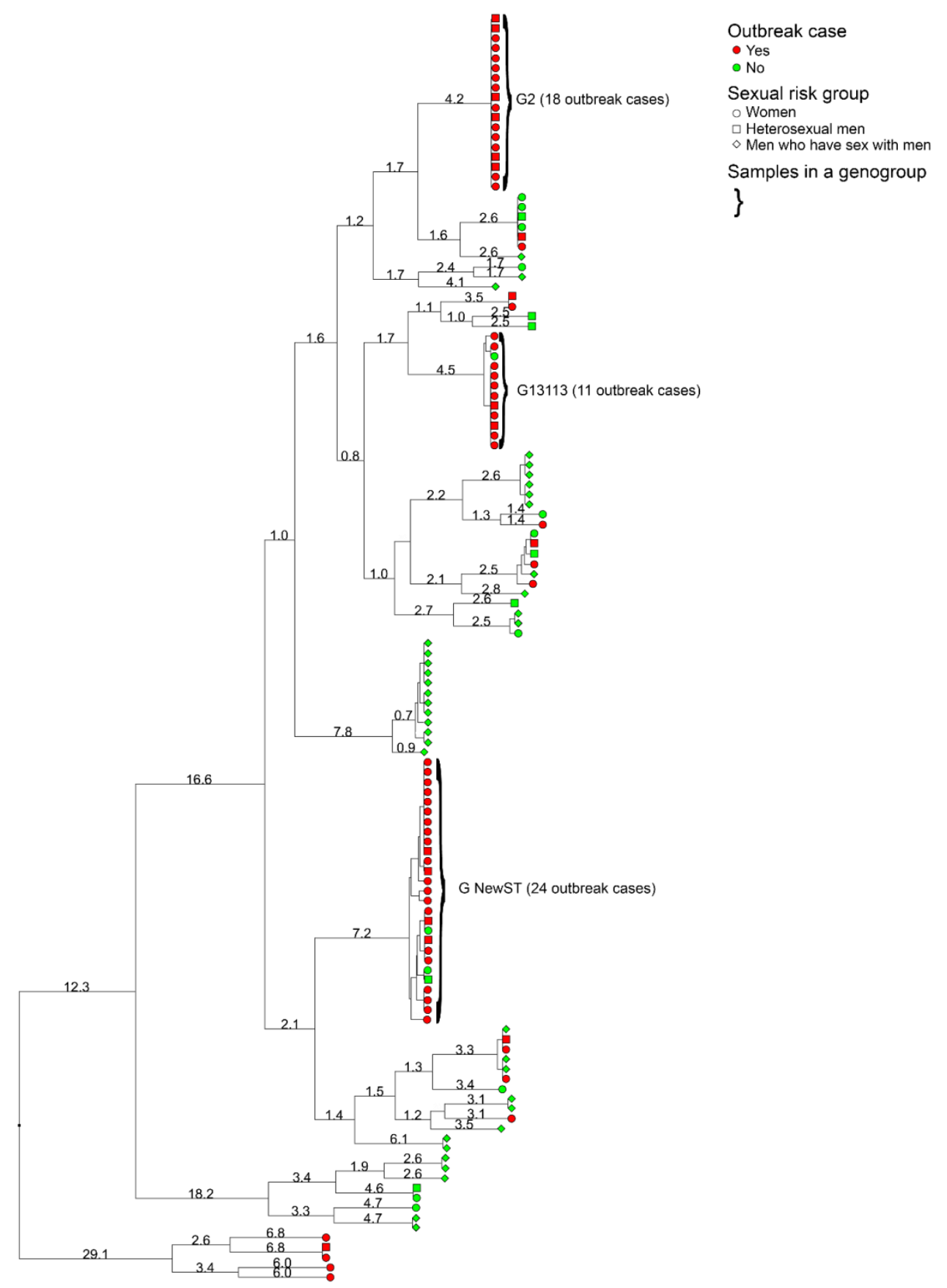

Figure 2 Dendogram of concatenated por $B$ and $t b p B$ sequences of outbreak (red) and reference (green) cases. Brackets indicate a genogroup and are named by the most abundant sequence type. The branch length depicts the percentage of dissimilarity. 


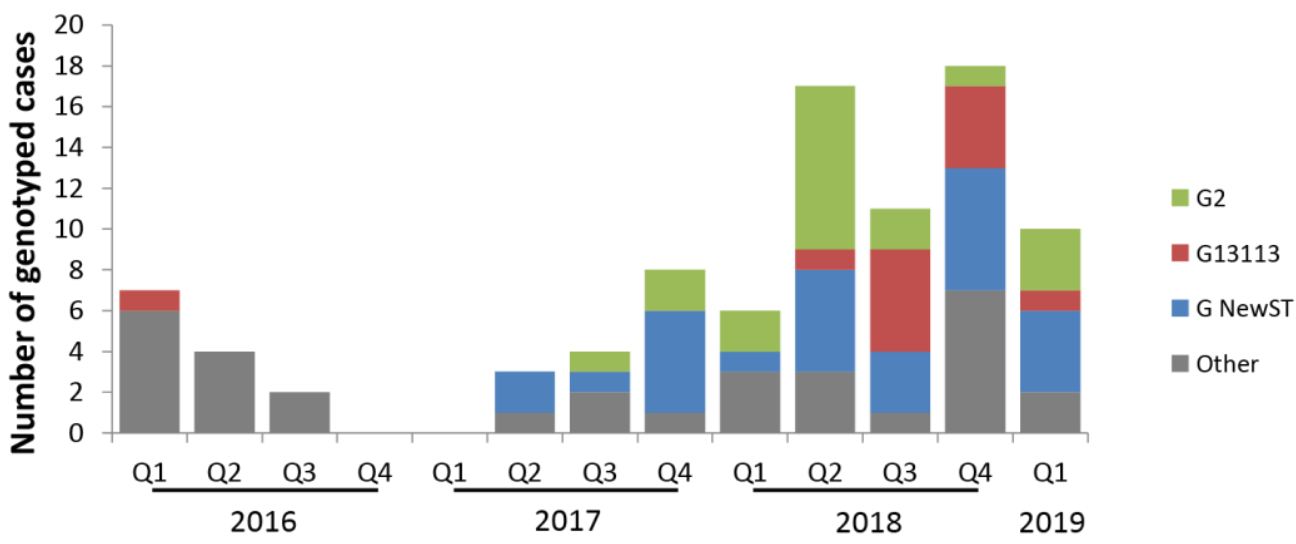

Figure 3 Epidemiological curve of genotyped samples of young heterosexuals in South-Limburg the Netherlands. Bars are coloured according to genogroup.

\section{Geographical mapping}

The four-digit postcode of patients per outbreak cluster were used to explore possibilities for targeted interventions. Figure 4 shows that the cases per outbreak cluster are quite scattered in South-Limburg. Despite the small numbers, geographical clustering analysis was performed using SatScan but no significant geographical clustering was observed (Supplemental table). 
A

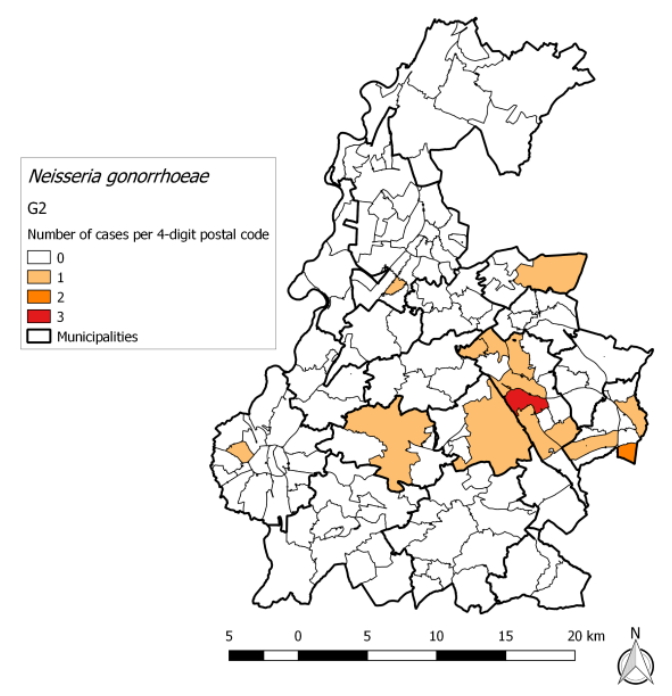

B

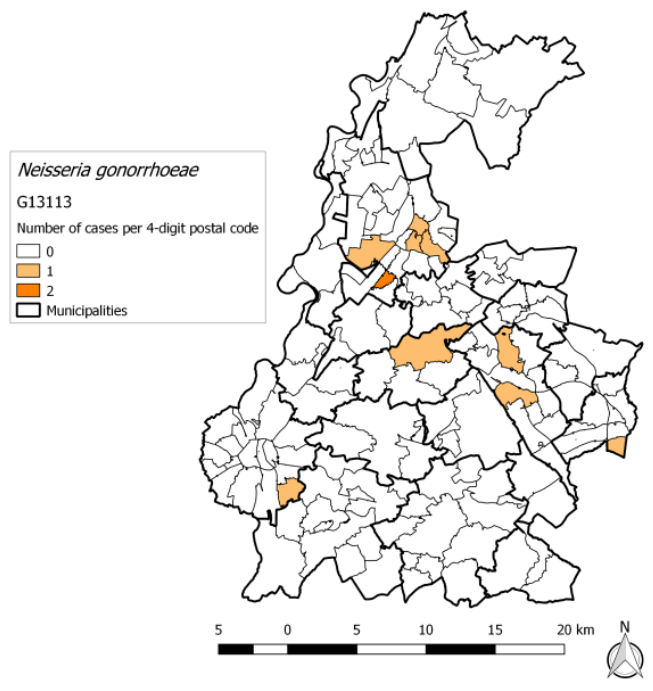

C

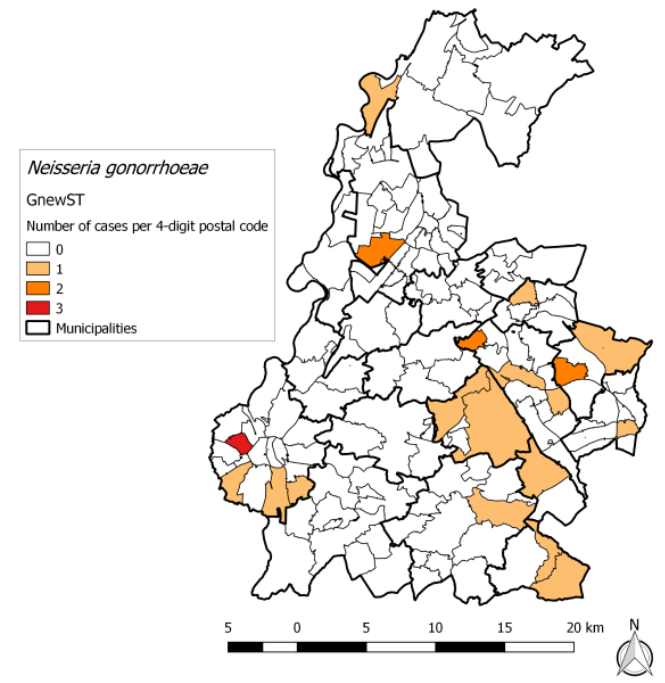

Figure 4 Geographical maps of cases per outbreak cluster.

\section{Association epidemiological characteristics with outbreak clusters}

As no clear geographical clustering was observed, other explanatory variables could provide insight in the epidemiology of the outbreak. Therefore, we analysed whether a significant association with sex, sex partner, CT co-infection, educational level, notified for an STI, symptoms, and ethnicity could be assessed. Fisher-exact tests showed that CT 
co-infection was significantly associated with cluster G NewST $(92 \%, p<0.03)$ (table 1). Higher number of sex partners was reported by heterosexual men in cluster G2 compared to the other clusters ( $p=0.03$, table 1$)$. In addition, women in cluster $G 2$ and G13113 report lower number of sex partners compared to heterosexual men in the respective cluster $(p=0.03$ and $p=0.02)$.

Table 1 Characteristics of the outbreak clusters. Data are reported as percentages (number of cases) except sex partners as mean (standard deviation).

\begin{tabular}{|c|c|c|c|c|}
\hline & & $\begin{array}{c}\text { G2 } \\
(n=18)\end{array}$ & $\begin{array}{l}G 13113 \\
(n=11) \\
\end{array}$ & $\begin{array}{l}\text { G NewST } \\
(\mathrm{n}=\mathbf{2 4})\end{array}$ \\
\hline \multirow[t]{2}{*}{ Sex } & Women & $66.7(12)$ & $81.8(9)$ & $83.3(20)$ \\
\hline & Men & $33.3(6)$ & $18.2(2)$ & $16.7(4)$ \\
\hline Sex partners reported by women & & $2.3( \pm 1.2)$ & $2.1( \pm 1.3)$ & $2.6( \pm 2.1)$ \\
\hline Sex partners reported by men & & $11.3( \pm 7.3)^{* a}$ & $5.5( \pm 0.7)^{b}$ & $2.8( \pm 2.4)$ \\
\hline \multirow[t]{2}{*}{ CT co-infection } & CT negative & $38.9(7)$ & $54.5(6)$ & $8.3(2)$ \\
\hline & CT positive & $61.1(11)$ & $45.5(5)$ & $91.7(22) *$ \\
\hline \multirow[t]{3}{*}{ Educational level } & Low/medium & $66.7(12)$ & $90.9(10)$ & $66.7(16)$ \\
\hline & High & $33.3(6)$ & $9.1(1)$ & $20.8(5)$ \\
\hline & Unknown & $0(0)$ & $0(0)$ & $12.5(3)$ \\
\hline \multirow[t]{3}{*}{ Notified for an STI } & Not notified & $77.8(14)$ & $45.5(5)$ & $62.5(15)$ \\
\hline & Notified & $22.2(4)$ & $45.5(5)$ & $37.5(9)$ \\
\hline & Unknown & $0.0(0)$ & $9.1(1)$ & $0.0(0)$ \\
\hline \multirow[t]{2}{*}{ Symptoms } & Asymptomatic & $16.7(3)$ & $27.3(3)$ & $45.8(11)$ \\
\hline & Symptomatic & $83.3(15)$ & $72.7(8)$ & $54.2(13)$ \\
\hline \multirow[t]{2}{*}{ Ethnicity } & Western & $88.9(16)$ & $81.8(9)$ & $95.8(23)$ \\
\hline & Not-western & $11.1(2)$ & $18.2(2)$ & $4.2(1)$ \\
\hline
\end{tabular}

$*=p<0.05$

${ }^{a}=$ Men report more sex partners than women $(p=0.03)$

${ }^{b}=$ Men report more sex partners than women $(p=0.02)$

\section{No introduction of NG by bridging}

The relatively high number of NG cases in the young heterosexual population could be the result of acquisition of NG by bridging with a high-risk population. As MSM, including bisexual men, are a risk group for acquiring a NG infection, bisexual men could introduce NG strains in the young heterosexual population. None of the cases of MSM, 
including bisexual men, clustered together with any of the three independent outbreaks (figure 2).

\section{Outbreak control measures}

Implementation of control measures to stop further transmission of NG within this population proved difficult, due to limited information on sexual partners and no obvious association with a geographical area. A prospective approach was taken in which an additional questionnaire is implemented, which will be collected upon return to the clinic for treatment or via telephone when empirically treated. This questionnaire covers detailed information of all sex partners, sexual practices per sex partner, and where and when did they meet their sex partner(s). Furthermore, prospective cultureindependent genotyping of NG-positive heterosexuals was implemented as it proved essential for outbreak clusters identification. This prospectively collected data of the additional questionnaire and genotyping can be used to perform network analyses for targeted interventions to control future NG outbreaks.

\section{Discussion}

Between October 2017 - March 2019 a three-fold higher number of genital NG-positive young heterosexuals per month was seen (4.5 per month) compared to January 2016 September 2017 (1.4 per month, considered as baseline), indicating an potential outbreak of NG among young heterosexuals while NG is mostly diagnosed in MSM. Therefore, culture-independent genotyping was performed and revealed three independent outbreak clusters of only heterosexual individuals. Using cultureindependent genotyping was essential to identify the three outbreak clusters as only 5/18 (G2), 2/11 (G13113), and 6/24 (G NewST) of cases had a cultured isolate available for typical culture-dependent genotyping. Analysis of these clusters showed a link with number of sex partners reported by men for cluster G2 and cluster G NewST with CT coinfection. The three independent strain clusters were not introduced by bridging with MSM sexual networks as these strains were not identified in samples of MSM. 
The three independent outbreak clusters (genogroups) were only found in heterosexual individuals. As these genogroups were not identified in samples of MSM, including bisexual men, our results make introduction of NG in the young heterosexual population by bridging from other sexual networks unlikely. However, bridging and mixed sexual risk groups have been described previously in strain clusters $[12,16]$. Some strains in each genogroup of this study were also found in a study by Choudhury et al. and were also associated with heterosexuals likely confirming the observed clusters in this study [12]. Choudhury et al. described significant differences in behaviour and demographics of individuals between strain clusters that could indicate localised transmission clusters. In this study number of sex partners reported by men and CT co-infection were significantly different between the clusters. Geographical mapping in this study did not reveal clear localised clusters possibly due to low absolute numbers. In contrast, Risley et al. did observe strain clusters to be associated with a geographical area and considered these as localised outbreaks and persisted shorter compared to strains that were not associated with a geographical area [13]. In this study only one strain (G13113) was observed in 2016 (considered pre-outbreak), while the other genogroups (G2 and G NewST) were not observed in 2016 (figure 3). Therefore, it is possible that the three clusters represent localised outbreaks in separate sexual networks but with similar epidemiological characteristics. For instance, the network could have an origin in dating app usage or attending the same school rather than location of residence.

Culture-independent molecular typing was essential for epidemiological analyses as in total 63/155 samples had a cultured isolate available. The outbreak clusters might not have been identified without this method, as only 5/18 (G2), 2/11 (G13113), and 6/24 (G NewST) of cases had a cultured isolate available for typical culture-dependent genotyping. Aside from the low sensitivity of NG culture, nucleic acid amplification tests (NAAT), which use non-culturable samples, are generally used for NG detection in routine diagnostics and are not always confirmed by culture [3,6]. This could lead to underestimation of linked individuals and delayed identification of outbreaks. Cultureindependent molecular typing on residual NAAT samples could provide links between individuals rapidly to inform STI clinics. 
Even though three independent outbreak clusters were identified, targeted control measures were difficult to setup because partner information from regular partner notification to identify transmission networks of the clusters was limited. Prospective genotyping in combination with intensive contact tracing could provide the necessary data to prevent transmission in sexual networks and control NG outbreaks. However, the highly feasible and cost-effective prospective genotyping could be implemented in many places and is available for future prospective studies.

Our study does have limitations. First, the outbreak cases were analysed and genotyped retrospectively, and therefore we were hampered in gathering additional information. Second, only genital NG cases of clients attending the STI clinic in South-Limburg were genotyped but not clients of general practitioners thereby potentially underestimating the magnitude of the outbreak clusters. However, groups at risk to acquire a NG infection are mainly tested at STI-clinics [10].

In conclusion, within the strong increase of NG cases in a short period of time, three independent outbreak clusters of closely related NG strains were identified using culture-free NG-MAST. This highlights the potential added value of culture-independent genotyping for outbreak investigations. As detailed partner information was limited and we did not observe clear associations within a geographical area, additional control measures were difficult to setup. Prospective culture-independent genotyping in combination with detailed epidemiological data could provide the data needed for additional control measures to halt NG transmission and control NG outbreaks. 


\section{References}

1. Newman L, Rowley J, Vander Hoorn S, Wijesooriya NS, Unemo M, Low N, et al. Global Estimates of the Prevalence and Incidence of Four Curable Sexually Transmitted Infections in 2012 Based on Systematic Review and Global Reporting. PLoS One. 2015;10(12):e0143304.

2. Quillin SJ, Seifert HS. Neisseria gonorrhoeae host adaptation and pathogenesis. Nat Rev Microbiol. 2018.

3. Bignell C, Unemo M, European STIGEB. 2012 European guideline on the diagnosis and treatment of gonorrhoea in adults. Int J STD AIDS. 2013;24(2):85-92.

4. World Health Organisation. Global action plan to control the spread and impact of antimicrobial resistance in Neisseria gonorrhoeae. 2012.

5. World Health Organisation. WHO guidelines for the Treatment of Neisseria gonorrhoeae. 2016.

6. Centers for Disease Control and Prevention. Recommendations for the laboratory-based detection of Chlamydia trachomatis and Neisseria gonorrhoeae--2014. MMWR Recomm Rep. 2014;63(RR-02):119.

7. Workowski KA, Bolan GA. Sexually transmitted diseases treatment guidelines, 2015. MMWR Recomm Rep. 2015;64(RR-03):1-137.

8. Chow EPF, Cornelisse VJ, Williamson DA, Priest D, Hocking JS, Bradshaw CS, et al. Kissing may be an important and neglected risk factor for oropharyngeal gonorrhoea: a cross-sectional study in men who have sex with men. Sex Transm Infect. 2019;95(7):516-21.

9. Chow EP, Tabrizi SN, Phillips S, Lee D, Bradshaw CS, Chen MY, et al. Neisseria gonorrhoeae Bacterial DNA Load in the Pharynges and Saliva of Men Who Have Sex with Men. J Clin Microbiol. 2016;54(10):2485-90.

10. Slurink IAL vAF, Heijne JCM, Op de Coul ELM, van Wees DA, Hoenderboom BM, Visser M, den Daas C, Woestenberg PJ, Göt HM, Nielen M, van Sighem Al, van Benthem BHB. Sexually transmitted infections in the Netherlands in 2018. National Institute for Public Health and Environment. 2019.

11. Town K, Bolt H, Croxford S, Cole M, Harris S, Field N, et al. Neisseria gonorrhoeae molecular typing for understanding sexual networks and antimicrobial resistance transmission: A systematic review. J Infect. 2018;76(6):507-14.

12. Choudhury B, Risley CL, Ghani AC, Bishop CJ, Ward H, Fenton KA, et al. Identification of individuals with gonorrhoea within sexual networks: a population-based study. Lancet. 2006;368(9530):139-46.

13. Risley CL, Ward H, Choudhury B, Bishop CJ, Fenton KA, Spratt BG, et al. Geographical and demographic clustering of gonorrhoea in London. Sex Transm Infect. 2007;83(6):481-7.

14. De Silva D, Peters J, Cole K, Cole MJ, Cresswell F, Dean G, et al. Whole-genome sequencing to determine transmission of Neisseria gonorrhoeae: an observational study. Lancet Infect Dis. 2016;16(11):1295-303.

15. Fifer H, Cole M, Hughes G, Padfield S, Smolarchuk C, Woodford N, et al. Sustained transmission of high-level azithromycin-resistant Neisseria gonorrhoeae in England: an observational study. Lancet Infect Dis. 2018.

16. Smolarchuk C, Wensley A, Padfield S, Fifer H, Lee A, Hughes G. Persistence of an outbreak of gonorrhoea with high-level resistance to azithromycin in England, November 2014May 2018. Euro Surveill. 2018;23(23).

17. Didelot X, Dordel J, Whittles LK, Collins C, Bilek N, Bishop CJ, et al. Genomic Analysis and Comparison of Two Gonorrhea Outbreaks. MBio. 2016;7(3).

18. Chisholm SA, Wilson J, Alexander S, Tripodo F, Al-Shahib A, Schaefer U, et al. An outbreak of highlevel azithromycin resistant Neisseria gonorrhoeae in England. Sex Transm Infect. 2016;92(5):365-7.

19. Cole MJ, Thomas DR, Chisholm SA, Abdullah AN, Birley H, Hosein I, et al. Molecular epidemiology of gonorrhoea in Wales (UK). Sex Transm Infect. 2013;89(3):267-72.

20. National Institute for Public Health and the Environment. Draaiboek Consult seksuele gezondheid. 2018.

21. van der Veer BM, Wolffs PF, Hoebe CJ, Dukers-Muijrers NH, van Alphen LB. Culture-free genotyping of Neisseria gonorrhoeae revealed distinct strains at different anatomical sites in a quarter of patients, the Netherlands, 2012 to 2016. Euro Surveill. 2018;23(50).

22. Chisholm SA, Unemo M, Quaye N, Johansson E, Cole MJ, Ison CA, et al. Molecular epidemiological typing within the European Gonococcal Antimicrobial Resistance Surveillance Programme reveals predominance of a multidrug-resistant clone. Euro Surveill. 2013;18(3). 
Potential Neisseria gonorrhoeae outbreak in young heterosexuals $(<25)$; culture-independent genotyping revealed three clusters, the Netherlands, October 2017 to March 2019

23. Bilek N, Martin IM, Bell G, Kinghorn GR, Ison CA, Spratt BG. Concordance between Neisseria gonorrhoeae genotypes recovered from known sexual contacts. J Clin Microbiol. 2007;45(11):35647.

24. QGIS Development Team 2019 QGIS Geographic Information System. Open Source Geospatial Foundation Project.

25. Jung I, Kulldorff M, Richard OJ. A spatial scan statistic for multinomial data. Stat Med. 2010;29(18):1910-8. 


\section{Supplemental file}

Supplemental table Geographical cluster detection analysis results using the multinomial model in SatScan.

\begin{tabular}{lllll}
\hline & Radius (km) & $\boldsymbol{n}$ & $\begin{array}{l}\text { RR each genogroup } \\
\text { (G2, G13113, G NewST) }\end{array}$ & $\boldsymbol{p}$-value \\
\hline $\begin{array}{l}\text { Cluster 1 } \\
\text { (Sittard-Geleen) }\end{array}$ & 7.88 & 10 & $(0.29,7.35,0.40)$ & 0.07 \\
$\begin{array}{l}\text { Cluster 2 } \\
\text { (Heerlen) }\end{array}$ & 4.89 & 5 & $(0.00,0.00,2.61)$ & 0.69 \\
$\begin{array}{l}\text { Cluster 3 } \\
\text { (Maastricht) }\end{array}$ & 2.73 & 6 & $(0.45,0.00,2.13)$ & 0.99 \\
$\begin{array}{l}\text { Cluster 4 } \\
\text { (Kerkrade) }\end{array}$ & 3.91 & 7 & $(2.47,0.64,0.29)$ & 1.00 \\
\hline $\begin{array}{l}n \text { is number of cases. RR is relative risk of the genogroup being inside the geographical area versus } \\
\text { outside. }\end{array}$ & & &
\end{tabular}





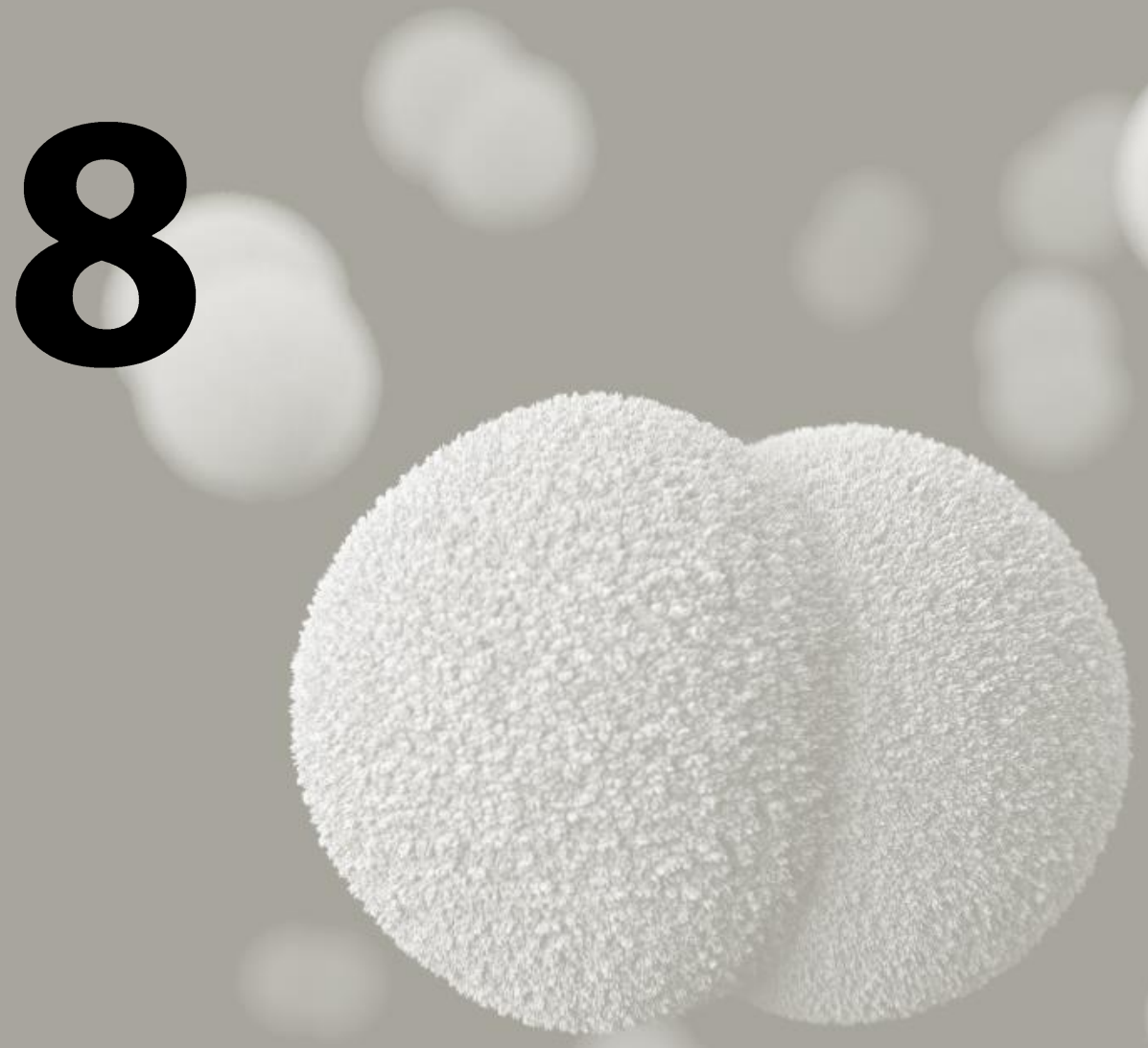




\section{Despite no ceftriaxone resistance in the Netherlands many Neisseria gonorrhoeae isolates have a mosaic penA gene}

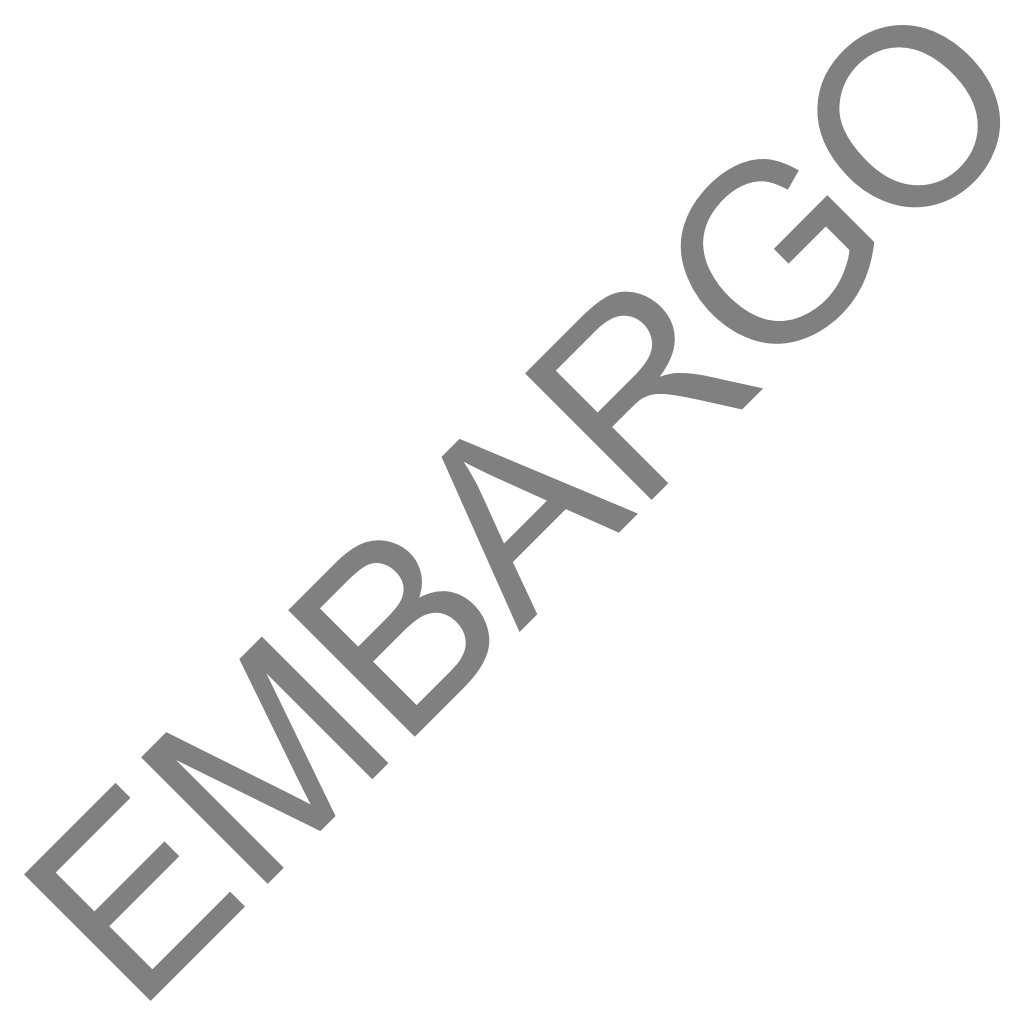

Brian M.J.W. van der Veer, Christian J.P.A. Hoebe, Nicole H.T.M. Duker-Muijrers,

Lieke B. van Alphen, Petra F.G. Wolffs 


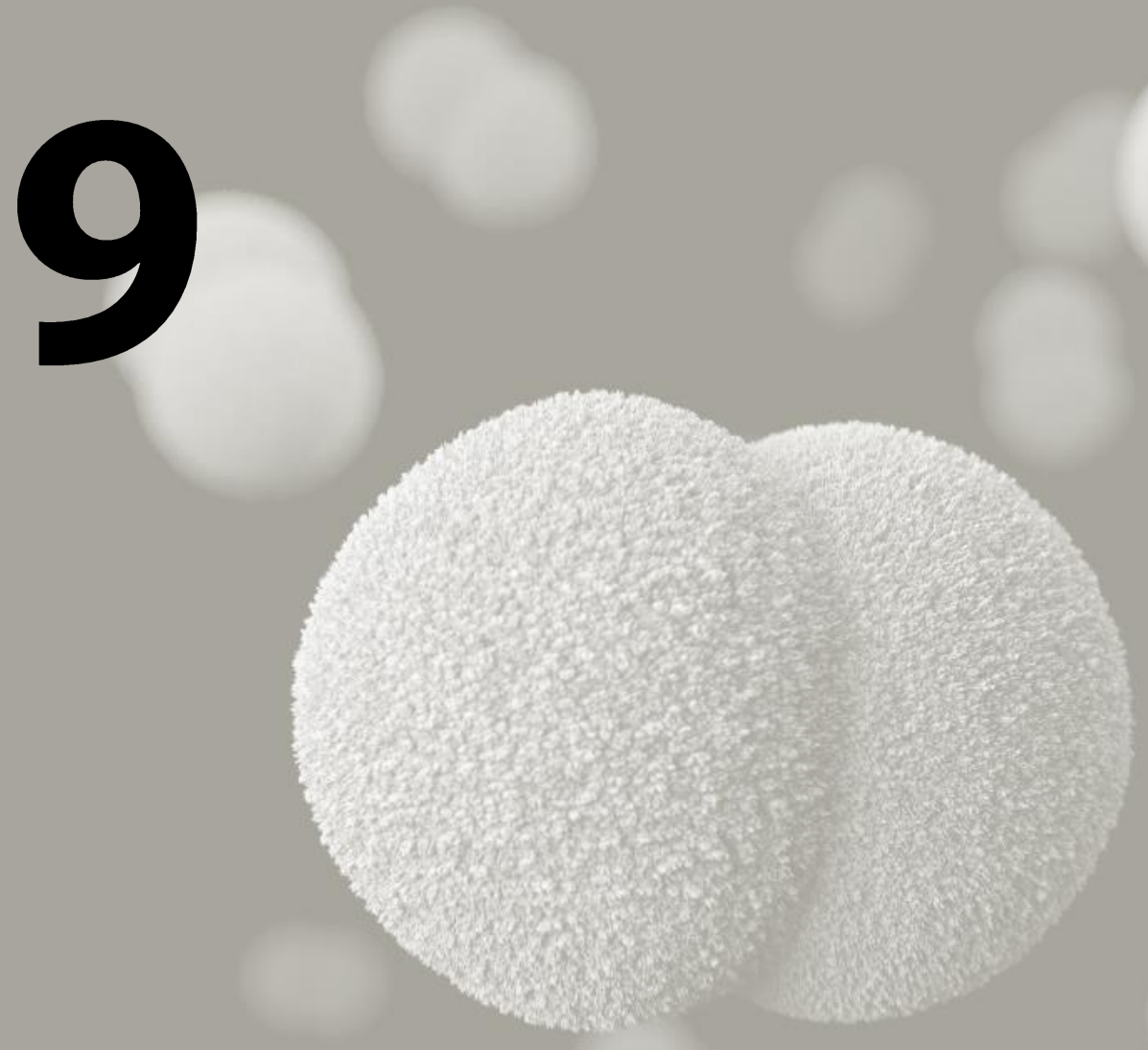


Summary and general discussion 
Neisseria gonorrhoeae (NG) is one of the most commonly diagnosed bacterial sexually transmitted infections (STI) worldwide [1]. The introduction of nucleic acid amplification tests (NAATs) revolutionized the ability to diagnose NG infections as non-invasive samples can be used [2]. Modern NAATs are highly sensitive and specific for NG detection and are automated. Hence, NAATs have replaced NG culture for routine screening diagnostics. However, current NAATs cannot determine the antimicrobial resistance (AMR) profile of a NG isolate [2]. As NG has developed resistance towards all classes of antibiotics, surveillance is critical to avoid untreatable NG [3,4]. Current surveillance requires a cultured NG isolate to monitor transmission and $\operatorname{AMR}[2,5,6]$. However, NG culture is complicated as it requires logistics and technical expertise because NG requires demanding nutritional and environmental conditions for growth [2]. Despite the improved diagnostics and screening strategies, the incidence of NG remains high $[1-3,7]$. Therefore, surveillance needs to be improved, and transmission dynamics and AMR acquisition of NG better understood. This in particular for extragenital sites of infection, especially the oropharynx, as they are considered a reservoir of AMR genes for NG $[8,9]$. This is because commensal Neisseria species are commonly present and easily exchange DNA with NG.

Several reports of high-level resistance in NG isolates prompted the World Health Organization (WHO) to develop a global action plan with recommendations to stop AMR and transmission of NG [3]. Strategies suggested included: effective diagnosis and NG control, systemic monitoring of treatment failures, strengthening of AMR surveillance, research into new molecular methods for surveillance, and new treatment options. With the studies in this thesis we aimed to gain further knowledge on effective diagnosis, transmission, improving surveillance, and further understanding of AMR in NG, including the potential pivotal role of the oropharynx and commensal Neisseria species as reservoir for resistance. In this final chapter, the main conclusions of the studies in this thesis are discussed in the light of existing literature. Furthermore, recommendations for future research are provided. 


\section{The clinical relevance of extra-genital NG infections}

The need for extra-genital testing is recognized for men who have sex with men (MSM) but not for women and heterosexual men $[2,5,10]$. Aside from MSM, extra-genital testing is only recommended for patients with an indication, such as a history of direct exposure by anal or oral sex or symptoms. This is linked to the difference in prevalence of extra-genital NG in MSM (3\% to $21 \%$ ) and heterosexual men and women (0\% to $3 \%$ ) $[11,12]$. Recent epidemiological studies demonstrate that oropharyngeal NG is common in MSM and women [13, 14]. Prevalence of oropharyngeal NG was $5.5 \%$ in MSM and $1.4 \%$ in women when routine universal testing was performed and shows that half of the oropharyngeal NG infections were oropharyngeal-only infections. An additional issue with extra-genital NG infections is the ongoing discussion of correct diagnoses of these infections [2, 15-17]. In first-generation NAATs false-positive results were the underlying issue. This has been solved in currently used modern NAATs, however these are not approved for extra-genital testing. Nevertheless, current laboratory standards recommend NAATs for the detection of extra-genital NG if a laboratory has established specifications for performance characteristics in accordance to present regulations [2]. Previous studies showed that the cobas $4800 \mathrm{CT} / \mathrm{NG}$ test could be used for anorectal swab testing and is highly specific $[15,18]$. Based on these results we argued that the cobas $4800 \mathrm{CT} / \mathrm{NG}$ test would perform well in oropharyngeal samples. In chapter $\mathbf{2}$, we assessed the validity of the cobas $4800 \mathrm{CT} / \mathrm{NG}$ test for detection of $C$. trachomatis (CT) and NG in oropharyngeal swabs. The cobas 4800 demonstrated very good performance for NG detection. The limit of detection, defined as $95 \%$ detection, was $6.65 \times 10^{2} \mathrm{CFU} / \mathrm{mL}$, but more importantly no cross-reactivity and high specificity (97.1\%) were observed. These results are in line with previous studies and demonstrate that NG is correctly diagnosed in extra-genital samples, including the oropharynx $[15,18]$. However, our study also raises a potential important issue as in theory up to $25 \%$ of oropharyngeal NG infections could be missed due to an average low NG bacterial load at this anatomical site. As there are no indications that the cobas $4800 \mathrm{CT} / \mathrm{NG}$ test is underperforming compared to other NAATs, this could be a common problem, likely across multiple 
platforms [19-21]. Therefore, more sensitive NAATs should be developed to avoid missed oropharyngeal NG infections that might otherwise be transmitted unnoticed.

The transmissibility of vaginal and extra-genital (anorectal and oropharyngeal) NG is not completely understood [9]. The load of a pathogen has been linked to transmission and clinical outcome in other STIs $[22,23]$. Therefore, the NG bacterial load could be linked to transmissibility and clinical outcome like symptoms. Previous studies have shown higher NG load in urethral infections of symptomatic men and anorectal infections of symptomatic MSM [24, 25]. Lower NG load was observed in oropharyngeal swabs of MSM compared to urethral and anorectal swabs. However, these studies were relatively small and no data exist of NG load in women in relation to symptoms. The NG load in a diverse population was determined in chapter 3, including genital and extra-genital samples. Loads were compared between sample material and sex, and associations of NG load with symptoms per sexual risk group to acquire an STI and sample material were tested. Our data demonstrate that men and women have a similar NG load in genital and extra-genital sites. This would imply that a NG infection is equally relevant in women and MSM. Our results also suggest that all anatomical sites may have a role in transmission because of the substantial NG load. Oropharyngeal NG infections might have a smaller role as the mean load is lower (16 to 25 -fold). Still, oropharyngeal NG infections are important as they are frequently single infections, mostly asymptomatic, and could be involved in AMR development of NG [4]. Hence, systematical extra-genital testing in routine diagnostics might reduce the incidence of NG. However, because of costs, systematical extra-genital testing will not be implemented in heterosexual men and women, as the prevalence is low $[11,12,26,27]$. In low-income countries this would be even less applicable. In many of those countries a syndromic approach based on symptoms linked with a NG infection is used $[2,28,29]$. As we demonstrate in chapter 3 that asymptomatic infections have sufficient load for transmission, these asymptomatic infections can cause more sequelae. For example, NG infections are linked with increased risk to acquire HIV [9]. 


\section{The need for improved NG surveillance}

Despite improved diagnostics and prevention efforts the incidence rate of NG infections remains high [1-3, 9]. Some key populations at risk to acquire a NG infection have been identified such as MSM, young people, and sex workers [3, 5, 10, 27]. Especially in these key populations, surveillance is essential to stop transmission and detect potential outbreaks [3]. Current surveillance relies on cultured isolates for genotyping to assess the genetic relatedness $[5,6]$. However, NG culture often fails as NG requires demanding nutritional and environmental conditions, leading to a low percentage of cultureconfirmed cases [30]. Hence, the WHO advocates in their global action plan to increase culture capacity and new molecular methods for surveillance [3]. Therefore, we believe that there is a need for culture-independent genotyping methods to overcome the issue with NG culture, thereby improving NG surveillance. We have developed a cultureindependent method based on the widely used NG multi-antigen sequence typing (NGMAST) in chapter 4, called "culture-free NG-MAST" [31-33]. Crucially, this method did not cross-react with any of the tested non-gonococcal Neisseria species. Therefore, the culture-free NG-MAST method can readily be used to genotype NG in clinical samples including extra-genital samples. In contrast, the culture-dependent NG-MAST cannot be used in uncultured extra-genital samples as it cross-reacts with commensal Neisseria species leading to wrongly assigned sequence types (ST) [34]. Furthermore, culture-free NG-MAST demonstrated a higher typing rate (94\%) compared to the culture-dependent method (62\%) in samples sent in for culture. Thus, the ability of including non-culturable and extra-genital samples, combined with the increased typing rate, can improved NG surveillance. However, one should note that (culture-free) NG-MAST is mainly applicable to identify transmission clusters [32]. Even though many NG-MAST STs are linked to resistance profiles in NG, this does not necessarily imply that the strain is phenotypically resistant [32, 35]. To date culture remains the 'golden-standard' for AMR surveillance [2]. 
In chapters 6 and 7, the potential added value of culture-free NG-MAST in surveillance and outbreak management was studied. In chapter 6, all samples $(n=196)$ subjected to routine culture between January 2017 till September 2018 from our STI clinic (South Limburg Public Health Service) were genotyped with culture-free NG-MAST. In total, $152 / 196(77 \%)$ were successfully genotyped and of the 44 samples that failed, 11 showed a possible mixed infection. Of the 196 samples, 115 were culture-positive which reflects an overall typing rate of $59 \%$. This is higher than the national average, likely as we only advise culture in case of high bacterial load [30]. Nevertheless, culture-free NGMAST demonstrates an overall higher typing rate (77\% versus $59 \%)$ and this rate is similar in culture-positive (80\%) versus negative samples (74\%). When stratified by sample material, the typing rate of urine samples did not differ between methods. However, culture-free NG-MAST vastly improved the typability of the other sample materials. In particular, the oropharyngeal site as only $6.3 \%(1 / 16)$ could be typed with culture-based methods, while with culture-free NG-MAST $81 \%(13 / 16)$ was genotyped. This improved typability demonstrated to be essential in a NG outbreak investigation (chapter 7). We noticed a potential outbreak of NG in the young $(<25)$ heterosexual population visiting our STI clinic between October 2017 till March 2019. A three-fold higher number of genital NG-positive young heterosexuals per month was seen (4.5 per month) compared to January 2016 - September 2017 (1.4 per month, considered as baseline). Using culture-free NG-MAST, three independent outbreak clusters were identified and this would not have been observed using culture-based methods. Still, implementation of control measures to stop further transmission of NG within the clusters proved difficult. This was due to limited information on sexual partners and no obvious association with a geographical area (residence). It could be possible that residence is not a good predictor because the individuals within a cluster could attend the same school or use the same dating app. Previous studies also show that molecular epidemiology, using NG-MAST, can help in identifying large clusters and newly emerging strains which we have also seen in both chapters 6 and 7 [36, 37]. Hence, we believe that culture-free NG-MAST has an added value in NG surveillance, which will be discussed in future directions. 


\section{Impact of multiple NG isolates in a patient}

As typically one isolate of a single anatomical site is characterized in surveillance, NG infections with multiple isolates like mixed infections are missed $[2,5,6]$. There are two types of multiple isolate infections in a patient; 1 ) distinct strains at separate anatomical sites, and 2) multiple isolates in one anatomical site (mixed infection). Both of these types were observed in previous studies but the frequency varies greatly between studies [31, 34, 38-44]. As many of these studies rely on culture, used less discriminatory methods than NG-MAST, or could not detect low abundant isolates $(<10 \%)$, we developed a targeted metagenomic method that can detect low abundant isolates and is highly discriminatory. We employed this to gain further knowledge on the frequency of multiple NG isolates in a patient. This metagenomic method is based on our highly discriminatory culture-free NG-MAST method [32]. In chapter 4 we included 81 MSM and 17 women with paired NG-positive samples from separate anatomical sites. In total, paired samples of 80/98 patients were genotyped in which distinct concurrent NG isolates at separate anatomical sites was observed in a quarter (20/80) of patients. This proportion is higher than most previous studies, likely because of study size, higher discriminatory method used, and inclusion of culture-negative samples [31, 34, 38, 39]. As the proportion of distinct NG isolates at separate anatomical sites was high, this needed further investigation to understand the high occurrence. The distinct NG strains at separate anatomical sites could be the result of mixed infections but only the dominant strain of an anatomical site was characterized. Our hypothesis is that also additional NG strains, previously undetected, could be present because of mixed infections. Therefore, we performed a follow-up study in chapter $\mathbf{5}$ of the samples of MSM with distinct NG strains at separate anatomical sites $(n=33)$ of chapter 4 . In chapter 5 a metagenomic genotyping method was developed by combining culturefree NG-MAST with next-generation sequencing and an analysis pipeline. Despite the selected MSM with distinct NG strains at separate anatomical sites, most samples (79\%, 25/33) show a single NG infection. Our study provides further evidence that mixed NG infections do occur but likely at low prevalence [40-44]. Possible explanations of the 
apparent low occurrence of mixed NG infections and implications of multiple NG isolates for surveillance are discussed below.

Based on our research in chapters $\mathbf{4}$ and $\mathbf{5}$ it appears that mixed NG infections are less frequent than distinct NG isolates at separate anatomical sites. There are several explanations like competition between NG strains, separate introduction of NG, and tissue tropism. In an murine infection model, fitness and competitive co-culture were studied of ceftriaxone resistant NG strains by Vincent et al. (2018) [45]. The authors report significant differences in fitness and in some competitive co-cultures with wildtype NG, no ceftriaxone resistant NG bacteria were recovered. This overgrowth of a more fit, in this case the wild-type NG, increased over time. As asymptomatic patients are tested at earliest 14 days after potential exposure, as described in the Dutch STI guidelines, this could explain a part of the low frequency of mixed NG infections [27]. The MSM in chapter 5 reported a median of 10 (6-18, inter quartile range) sex partners in the previous six months. Hence, they could be exposed to NG at different points in time. Combined with competition between NG isolates, an established infection of NG might prevent colonization of a newly introduced NG isolate. However, reporting multiple sex partners does not reflect multiple potential exposures of NG at an anatomical site. Studies that assessed sexual behavior show that sexual practices per sex partner can vary greatly, including condom use and type of sexual practice per sex partner [46, 47]. Lastly, tissue tropism might contribute to the low occurrence of mixed infections as some isolates might infect certain anatomical sites poorly. However, many cell adherence proteins can undergo phase variation to mediate and adept adherence to various human cells [48-50]. Also, NG can adept to different environmental conditions by an array of responsive transcriptional factors [51]. Therefore, NG isolates should be able to adjust to an anatomical site and likely does not explain the low frequency of mixed infections. Still, these mixed infections do occur and could be relevant in treatment as a potential resistant NG isolate in a mixed infection might lead to treatment failure. Also, mixed infections could be relevant for AMR development as AMR genes can be exchanged between NG isolates [9]. As discussed before, typically one isolate of a single anatomical site is characterized in surveillance $[2,5,6]$. Therefore, infections with 
multiple NG isolates would be missed in the current surveillance strategies possibly allowing dissemination of resistant NG strains. Hence, distinct NG isolates at separate anatomical sites should be included in surveillance as these infections occur frequently.

\section{The oropharyngeal site as a reservoir for antimicrobial resistance}

Mosaic genes can occur in the genome of NG and lead to resistance towards various antimicrobials $[4,8]$. As ceftriaxone is the current first-line empirical treatment of NG and not many treatment options are left, AMR research is essential to avoid untreatable NG $[3,28]$. Mosaic penA genes can results in ceftriaxone resistance $[4,9]$. These mosaic genes are considered to have emerged by DNA recombination with DNA of commensal Neisseria species that commonly reside the oropharynx. Hence, the oropharynx is considered to be a reservoir for AMR genes of NG $[52,53]$. In a study by lgawa et al. (2018), in vitro recombination of NG with N. cinerea DNA has been demonstrated [54]. However, this does not necessarily reflect the process in vivo as for example the regulation of DNA donation is unclear [55]. Mostly N. cinerea and N. subflava group are involved in mosaic penA genes in NG. Therefore, the presence of $N$. cinerea and $N$. subflava in the oropharynx during a NG infection would be a prerequisite for recombination. To study the presence of Neisseria species and potential recombination in NG during an oropharyngeal infection, we developed a metagenomics method that uses nanopore sequencing of amplicons to characterize the penA genes and their flanking regions in chapter $\mathbf{8}$. The flanking regions of the $p e n A$ gene were used to identify the species. Hence, we could compare penA genes and identify potential recombination events. In this study 64 NG-positive oropharyngeal samples of MSM were characterized of which 61 were penA PCR positive. In almost half of these samples $(28 / 61)$ a NG contig was observed and $8 / 28$ were mosaic. All except one had amino acid substitutions 1312M and V316T linked to ceftriaxone resistance [4]. We demonstrate that recombination during a NG infection could be possible as in all samples (61/61) N. subflava and in more than half of the oropharyngeal samples (35/61) N. cinerea were present. In addition, in all samples with a mosaic penA gene in NG, the mosaic part of NG was similar to either $N$. cinerea or $N$. subflava was seen. Though resistance is low in the Netherlands, in $29 \%(8 / 28)$ of NG contigs a mosaic penA was observed and this is in line 
with the recent study of de Laat et al. (2019) [30,56]. Recent epidemiological studies in other countries report mosaic penA gene in $2.3 \%$ to $27 \%$ of tested isolates [57-61]. Worrisome is that the proportion of strains with mosaic penA appears to be increasing $[59,61]$. Also, A501 mutations in non-mosaic penA, linked to ceftriaxone resistance, is commonly reported [56, 57, 59]. In our study from January 2017 to June 2018, only one NG isolate has the mutation A501V and is non-mosaic. To summarize this part, it is clear that commensal Neisseria species commonly reside the oropharynx also during a NG infection. As Neisseria species readily exchange DNA and the mosaic part of NG was similar to commensal Neisseria species, recombination in vivo could have a pivotal role in AMR development in NG $[62,63]$. Still, there are many unknowns like efficiency in DNA donation and uptake, fitness cost of recombination, AMR in commensal Neisseria species, and potential killing of NG by commensal Neisseria species DNA [64, 65].

\section{Concluding remarks and future perspectives}

Despite many efforts to detect and treat NG infections the incidence remains high. Guidelines recognize the need for extra-genital testing in MSM and specific key populations $[2,5,10]$. In this thesis we observed many single oropharyngeal NG infections which are mostly asymptomatic. Though the cobas $4800 \mathrm{CT} / \mathrm{NG}$ test demonstrates very good performance to detect NG in oropharyngeal samples, in theory, up to $25 \%$ could be missed. Because there is no indication that the cobas 4800 underperforms compared to other NAATs, more sensitive tests are needed. Future studies should address the impact of this potential issue. For example, repeated testing of NG-negative oropharyngeal could be performed to estimate the initially missed infections.

One of the goals of this thesis was to improve surveillance of NG. We demonstrated that culture-independent typing was essential to identify NG outbreak clusters. As mentioned several times, diagnostics and surveillance has vastly improved in the past decades but NG remains a global issue. We believe that a prospective typing in combination with intensive contact tracing could aid to limit NG transmission and provide early detection of outbreaks. With this data, sexual networks should readily be 
identified for a targeted intervention like intensified testing and counseling. With unlimited resources, ideally, all NG positive samples should be typed to have the highest resolution to identify clusters. Together with metadata from the public health services an interactive NG infection monitoring tool for clinicians, epidemiologists, and microbiologist could be created. In this tool a warning system could be implemented to highlight regions or populations that might need additional attention. For instance, a potential NG outbreak could be early identified to take action to stop transmission. Unfortunately, resources are limited and the estimated costs for typing are $€ 40$ using culture-free NG-MAST, including a technician [27]. A pilot study using the proposed approach should be conducted that (hopefully) shows clusters to allow interventions that result in a decrease in incidence of NG.

Gonorrhea is a major public health challenge today, not only because of the high incidence but also because of increasing resistance. Based on previous results and the results in chapter $\mathbf{8}$ commensal Neisseria species appear to have a pivotal role in AMR development of NG. However, data on commensal Neisseria species is limited. Hence, future research should focus more on the commensal Neisseria rather than only target NG. For instance, little is known about the minimal inhibitory concentration (MIC) values in these species. This is particularly relevant for AMR in NG because commensal Neisseria could become resistant after multiple exposures to antibiotics and could exchange the AMR genes with NG. Therefore, it is important to better understand the dynamics of the commensal Neisseria. For example, a longitudinal study in which individuals are sampled regularly could provide further knowledge. Thereby addressing, but not limited to, the composition of the oropharyngeal Neisseria species, mosaic genes, MIC values, and whole-genome sequences of isolates.

Though there is a lack of novel antibiotics for treatment of NG, we could improve care and limit ceftriaxone usage to potentially limit AMR development. By using point-of-care tests or new NAATs that can also identify NG isolates that are still susceptible to previously used antibiotics like spectinomycin or ciprofloxacin, and treat the patient with one of these antibiotics. 
In summary, with the introduction of NAATs the diagnoses of an infection with NG became more sensitive and with antibiotics the treatment became easier. However, NG proved to be a highly adaptive pathogen that is increasingly resistant to all classes of antibiotics. Therefore, surveillance programs were established to monitor and prevent resistant NG. Still, much remains to be understood like the role of commensal Neisseria species and the persistent high incidence of NG globally. The research presented in this thesis elucidates a part of the puzzle to understand transmission dynamic but also provide tools to improve NG surveillance. 


\section{References}

1. Rowley J, Vander Hoorn S, Korenromp E, Low N, Unemo M, Abu-Raddad LJ, et al. Chlamydia, gonorrhoea, trichomoniasis and syphilis: global prevalence and incidence estimates, 2016. B World Health Organ. 2019;97(8):548-+.

2. Centers for Disease Control and Prevention. Recommendations for the laboratory-based detection of Chlamydia trachomatis and Neisseria gonorrhoeae--2014. MMWR Recomm Rep. 2014;63(RR-02):119.

3. World Health Organisation. Global action plan to control the spread and impact of antimicrobial resistance in Neisseria gonorrhoeae. 2012.

4. Unemo M, Golparian D, Eyre DW. Antimicrobial Resistance in Neisseria gonorrhoeae and Treatment of Gonorrhea. Methods Mol Biol. 2019;1997:37-58.

5. Workowski KA, Bolan GA. Sexually transmitted diseases treatment guidelines, 2015. MMWR Recomm Rep. 2015;64(RR-03):1-137.

6. European Centre for Disease Prevention and Control (ECDC). Gonococcal antimicrobial susceptibility surveillance in Europe. 2015.

7. Centers for Disease Control and Prevention. Sexually Transmitted Disease Surveillance 2014. 2014.

8. Unemo M, Shafer WM. Antimicrobial resistance in Neisseria gonorrhoeae in the 21st century: past, evolution, and future. Clin Microbiol Rev. 2014;27(3):587-613.

9. Unemo M, Seifert HS, Hook EW, 3rd, Hawkes S, Ndowa F, Dillon JR. Gonorrhoea. Nat Rev Dis Primers. 2019;5(1):79.

10. LeFevre ML. Screening for Chlamydia and gonorrhea: U.S. Preventive Services Task Force recommendation statement. Ann Intern Med. 2014;161(12):902-10.

11. Dukers-Muijrers NH, Schachter J, van Liere GA, Wolffs PF, Hoebe CJ. What is needed to guide testing for anorectal and pharyngeal Chlamydia trachomatis and Neisseria gonorrhoeae in women and men? Evidence and opinion. BMC Infect Dis. 2015;15:533.

12. Garner AL, Schembri G, Cullen T, Lee V. Should we screen heterosexuals for extra-genital chlamydial and gonococcal infections? Int J STD AIDS. 2015;26(7):462-6.

13. van Liere G, Dukers-Muijrers N, Kuizenga-Wessel S, Gotz HM, Hoebe C. What Is the Optimal Testing Strategy for Oropharyngeal Neisseria gonorrhoeae in Men Who Have Sex With Men? Comparing Selective Testing Versus Routine Universal Testing From Dutch Sexually Transmitted Infection Clinic Data (2008-2017). Clin Infect Dis. 2020;71(4):944-51.

14. Van Liere GAFS D-MN, Kuizenga Wessel S, Wolffs PF, Hoebe CJPA. Routine universal testing versus selective or incidental testing for oropharyngeal Neisseria gonorrhoeae in women in the Netherlands: a retrospective cohort study. Lancet Infect Dis. 2020 in press.

15. Tabrizi SN, Unemo M, Limnios AE, Hogan TR, Hjelmevoll SO, Garland SM, et al. Evaluation of six commercial nucleic acid amplification tests for detection of Neisseria gonorrhoeae and other Neisseria species. J Clin Microbiol. 2011;49(10):3610-5.

16. Marangoni A, Foschi C, Nardini P, Compri M, Cevenini R. Evaluation of the Versant CT/GC DNA 1.0 assay (KPCR) for the detection of extra-genital Chlamydia trachomatis and Neisseria gonorrhoeae infections. PLoS One. 2015;10(3):e0120979.

17. Palmer HM, Mallinson H, Wood RL, Herring AJ. Evaluation of the specificities of five DNA amplification methods for the detection of Neisseria gonorrhoeae. J Clin Microbiol. 2003;41(2):835-7.

18. Geelen TH, Rossen JW, Beerens AM, Poort L, Morre SA, Ritmeester WS, et al. Performance of cobas(R) 4800 and m2000 real-time assays for detection of Chlamydia trachomatis and Neisseria gonorrhoeae in rectal and self-collected vaginal specimen. Diagn Microbiol Infect Dis. 2013;77(2):101-5.

19. Taylor SN, Liesenfeld O, Lillis RA, Body BA, Nye M, Williams J, et al. Evaluation of the Roche cobas(R) CT/NG test for detection of Chlamydia trachomatis and Neisseria gonorrhoeae in male urine. Sex Transm Dis. 2012;39(7):543-9.

20. Van Der Pol B, Liesenfeld O, Williams JA, Taylor SN, Lillis RA, Body BA, et al. Performance of the cobas CT/NG test compared to the Aptima AC2 and Viper CTQ/GCQ assays for detection of Chlamydia trachomatis and Neisseria gonorrhoeae. J Clin Microbiol. 2012;50(7):2244-9. 
21. Chernesky M, Jang D, Gilchrist J, Hatchette T, Poirier A, Flandin JF, et al. Head-to-head comparison of second-generation nucleic acid amplification tests for detection of Chlamydia trachomatis and Neisseria gonorrhoeae on urine samples from female subjects and self-collected vaginal swabs. J Clin Microbiol. 2014;52(7):2305-10.

22. Pedraza MA, del Romero J, Roldan F, Garcia S, Ayerbe MC, Noriega AR, et al. Heterosexual transmission of HIV-1 is associated with high plasma viral load levels and a positive viral isolation in the infected partner. J Acquir Immune Defic Syndr. 1999;21(2):120-5.

23. Mavilia MG, Wu GY. Mechanisms and Prevention of Vertical Transmission in Chronic Viral Hepatitis. J Clin Transl Hepato. 2017;5(2):119-29.

24. Priest D, Ong JJ, Chow EP, Tabrizi S, Phillips S, Bissessor M, et al. Neisseria gonorrhoeae DNA bacterial load in men with symptomatic and asymptomatic gonococcal urethritis. Sex Transm Infect. 2017.

25. Bissessor M, Tabrizi SN, Fairley CK, Danielewski J, Whitton B, Bird S, et al. Differing Neisseria gonorrhoeae bacterial loads in the pharynx and rectum in men who have sex with men: implications for gonococcal detection, transmission, and control. J Clin Microbiol. 2011;49(12):4304-6.

26. Koedijk FD, van Bergen JE, Dukers-Muijrers NH, van Leeuwen AP, Hoebe CJ, van der Sande MA, et al. The value of testing multiple anatomic sites for gonorrhoea and chlamydia in sexually transmitted infection centres in the Netherlands, 2006-2010. Int J STD AIDS. 2012;23(9):626-31.

27. National Institute for Public Health and the Environment. Draaiboek Consult seksuele gezondheid. 2018.

28. World Health Organisation. WHO guidelines for the Treatment of Neisseria gonorrhoeae. 2016.

29. Ndowa FJ, Francis JM, Machiha A, Faye-Kette H, Fonkoua MC. Gonococcal antimicrobial resistance: perspectives from the African region. Sex Transm Infect. 2013;89 Suppl 4:iv11-5.

30. Staritsky LE vAF, Visser M, Op de Coul ELM, Heijne JCM, Götz HM, Nielen M, van Sighem Al, van Benthem BHB. Sexually transmitted infections in the Netherlands in 2019. National Institute for Public Health and Environment. 2020.

31. Carannante A, Ghisetti V, Dal Conte I, Gregori G, Stella ML, Vacca P, et al. Molecular characterization of Neisseria gonorrhoeae on non-cultured specimens from multiple anatomic sites. Ann Ist Super Sanita. 2017;53(3):213-7.

32. Martin IM, Ison CA, Aanensen DM, Fenton KA, Spratt BG. Rapid sequence-based identification of gonococcal transmission clusters in a large metropolitan area. J Infect Dis. 2004;189(8):1497-505.

33. Unemo M, Dillon JA. Review and international recommendation of methods for typing neisseria gonorrhoeae isolates and their implications for improved knowledge of gonococcal epidemiology, treatment, and biology. Clin Microbiol Rev. 2011;24(3):447-58.

34. Whiley DM, Goire N, Ray ES, Limnios A, Lambert SB, Nissen MD, et al. Neisseria gonorrhoeae multiantigen sequence typing using non-cultured clinical specimens. Sexually Transmitted Infections. 2010;86(1):51-5.

35. Chisholm SA, Unemo M, Quaye N, Johansson E, Cole MJ, Ison CA, et al. Molecular epidemiological typing within the European Gonococcal Antimicrobial Resistance Surveillance Programme reveals predominance of a multidrug-resistant clone. Euro Surveill. 2013;18(3).

36. Risley CL, Ward H, Choudhury B, Bishop CJ, Fenton KA, Spratt BG, et al. Geographical and demographic clustering of gonorrhoea in London. Sex Transm Infect. 2007;83(6):481-7.

37. Choudhury B, Risley CL, Ghani AC, Bishop CJ, Ward H, Fenton KA, et al. Identification of individuals with gonorrhoea within sexual networks: a population-based study. Lancet. 2006;368(9530):139-46.

38. Pond MJ, Hall CL, Miari VF, Cole M, Laing KG, Jagatia H, et al. Accurate detection of Neisseria gonorrhoeae ciprofloxacin susceptibility directly from genital and extragenital clinical samples: towards genotype-guided antimicrobial therapy. J Antimicrob Chemother. 2016;71(4):897-902.

39. De Silva D, Peters J, Cole K, Cole MJ, Cresswell F, Dean G, et al. Whole-genome sequencing to determine transmission of Neisseria gonorrhoeae: an observational study. Lancet Infect Dis. 2016;16(11):1295-303.

40. Kolader ME, Dukers NH, van der Bij AK, Dierdorp M, Fennema JS, Coutinho RA, et al. Molecular epidemiology of Neisseria gonorrhoeae in Amsterdam, The Netherlands, shows distinct heterosexual and homosexual networks. J Clin Microbiol. 2006;44(8):2689-97.

41. Lynn F, Hobbs MM, Zenilman JM, Behets FM, Van Damme K, Rasamindrakotroka A, et al. Genetic typing of the porin protein of Neisseria gonorrhoeae from clinical noncultured samples for strain characterization and identification of mixed gonococcal infections. J Clin Microbiol. 2005;43(1):36875. 
42. Martin IM, Ison CA. Detection of mixed infection of Neisseria gonorrhoeae. Sex Transm Infect. 2003;79(1):56-8.

43. Trembizki E, Doyle C, Buckley C, Jennison A, Smith H, Bates J, et al. Estimating the prevalence of mixed-type gonococcal infections in Queensland, Australia. Sex Health. 2015;12(5):439-44.

44. Goire N, Kundu R, Trembizki E, Buckley C, Hogan TR, Lewis DA, et al. Mixed gonococcal infections in a high-risk population, Sydney, Australia 2015: implications for antimicrobial resistance surveillance? J Antimicrob Chemother. 2017;72(2):407-9.

45. Vincent LR, Kerr SR, Tan Y, Tomberg J, Raterman EL, Hotopp JCD, et al. In Vivo-Selected Compensatory Mutations Restore the Fitness Cost of Mosaic penA Alleles That Confer Ceftriaxone Resistance in Neisseria gonorrhoeae. Mbio. 2018;9(2).

46. Arnold MP, Struthers H, McIntyre J, Lane T. Contextual correlates of per partner unprotected anal intercourse rates among MSM in Soweto, South Africa. AIDS Behav. 2013;17 Suppl 1:S4-11.

47. Chow EPF, Cornelisse VJ, Williamson DA, Priest D, Hocking JS, Bradshaw CS, et al. Kissing may be an important and neglected risk factor for oropharyngeal gonorrhoea: a cross-sectional study in men who have sex with men. Sex Transm Infect. 2019;95(7):516-21.

48. Jonsson AB, Ilver D, Falk P, Pepose J, Normark S. Sequence changes in the pilus subunit lead to tropism variation of Neisseria gonorrhoeae to human tissue. Mol Microbiol. 1994;13(3):403-16.

49. Edwards JL, Butler EK. The Pathobiology of Neisseria gonorrhoeae Lower Female Genital Tract Infection. Front Microbiol. 2011;2:102.

50. Hill SA, Masters TL, Wachter J. Gonorrhea - an evolving disease of the new millennium. Microb Cell. 2016;3(9):371-89.

51. Quillin SJ, Seifert HS. Neisseria gonorrhoeae host adaptation and pathogenesis. Nat Rev Microbiol. 2018.

52. Ameyama S, Onodera S, Takahata M, Minami S, Maki N, Endo K, et al. Mosaic-like structure of penicillin-binding protein 2 Gene (penA) in clinical isolates of Neisseria gonorrhoeae with reduced susceptibility to cefixime. Antimicrob Agents Chemother. 2002;46(12):3744-9.

53. Ito M, Deguchi T, Mizutani KS, Yasuda M, Yokoi S, Ito S, et al. Emergence and spread of Neisseria gonorrhoeae clinical isolates harboring mosaic-like structure of penicillin-binding protein 2 in Central Japan. Antimicrob Agents Chemother. 2005;49(1):137-43.

54. Igawa G, Yamagishi Y, Lee KI, Dorin M, Shimuta K, Suematsu H, et al. Neisseria cinerea with High Ceftriaxone MIC Is a Source of Ceftriaxone and Cefixime Resistance-Mediating penA Sequences in Neisseria gonorrhoeae. Antimicrob Agents Chemother. 2018;62(3).

55. Hamilton HL, Dominguez NM, Schwartz KJ, Hackett KT, Dillard JP. Neisseria gonorrhoeae secretes chromosomal DNA via a novel type IV secretion system. Mol Microbiol. 2005;55(6):1704-21.

56. de Laat MM, Wind CM, Bruisten SM, Dierdorp M, de Vries HJC, Schim van der Loeff MF, et al. Ceftriaxone Reduced Susceptible Neisseria gonorrhoeae in the Netherlands, 2009 to 2017: From PenA Mosaicism to A501T/V Nonmosaicism. Sex Transm Dis. 2019;46(9):594-601.

57. Golparian D, Bazzo ML, Golfetto L, Gaspar PC, Schorner MA, Schwartz Benzaken A, et al. Genomic epidemiology of Neisseria gonorrhoeae elucidating the gonococcal antimicrobial resistance and lineages/sublineages across Brazil, 2015-16. J Antimicrob Chemother. 2020.

58. Boiko I, Golparian D, Jacobsson S, Krynytska I, Frankenberg A, Shevchenko T, et al. Genomic epidemiology and antimicrobial resistance determinants of Neisseria gonorrhoeae isolates from Ukraine, 2013-2018. APMIS. 2020;128(7):465-75.

59. Lan PT, Golparian D, Ringlander J, Van Hung L, Van Thuong N, Unemo M. Genomic analysis and antimicrobial resistance of Neisseria gonorrhoeae isolates from Vietnam in 2011 and 2015-16. J Antimicrob Chemother. 2020;75(6):1432-8.

60. Attram N, Agbodzi B, Dela H, Behene E, Nyarko EO, Kyei NNA, et al. Antimicrobial resistance (AMR) and molecular characterization of Neisseria gonorrhoeae in Ghana, 2012-2015. PLoS One. 2019;14(10):e0223598.

61. Lee H, Suh YH, Lee S, Kim YK, Han MS, Bae HG, et al. Emergence and Spread of CephalosporinResistant Neisseria gonorrhoeae with Mosaic penA Alleles, South Korea, 2012-2017. Emerg Infect Dis. 2019;25(3):416-24.

62. Saika T, Nishiyama T, Kanayama A, Kobayashi I, Nakayama H, Tanaka M, et al. Comparison of Neisseria gonorrhoeae isolates from the genital tract and pharynx of two gonorrhea patients. J Infect Chemother. 2001;7(3):175-9. 


\section{Chapter 9}

63. Eyre DW, Sanderson ND, Lord E, Regisford-Reimmer N, Chau K, Barker L, et al. Gonorrhoea treatment failure caused by a Neisseria gonorrhoeae strain with combined ceftriaxone and highlevel azithromycin resistance, England, February 2018. Euro Surveill. 2018;23(27).

64. So M, Rendon MA. Tribal warfare: Commensal Neisseria kill pathogen Neisseria gonorrhoeae using its DNA. Microb Cell. 2019;6(12):544-6.

65. Kim WJ, Higashi D, Goytia M, Rendon MA, Pilligua-Lucas M, Bronnimann M, et al. Commensal Neisseria Kill Neisseria gonorrhoeae through a DNA-Dependent Mechanism. Cell Host Microbe. 2019;26(2):228-39 e8. 



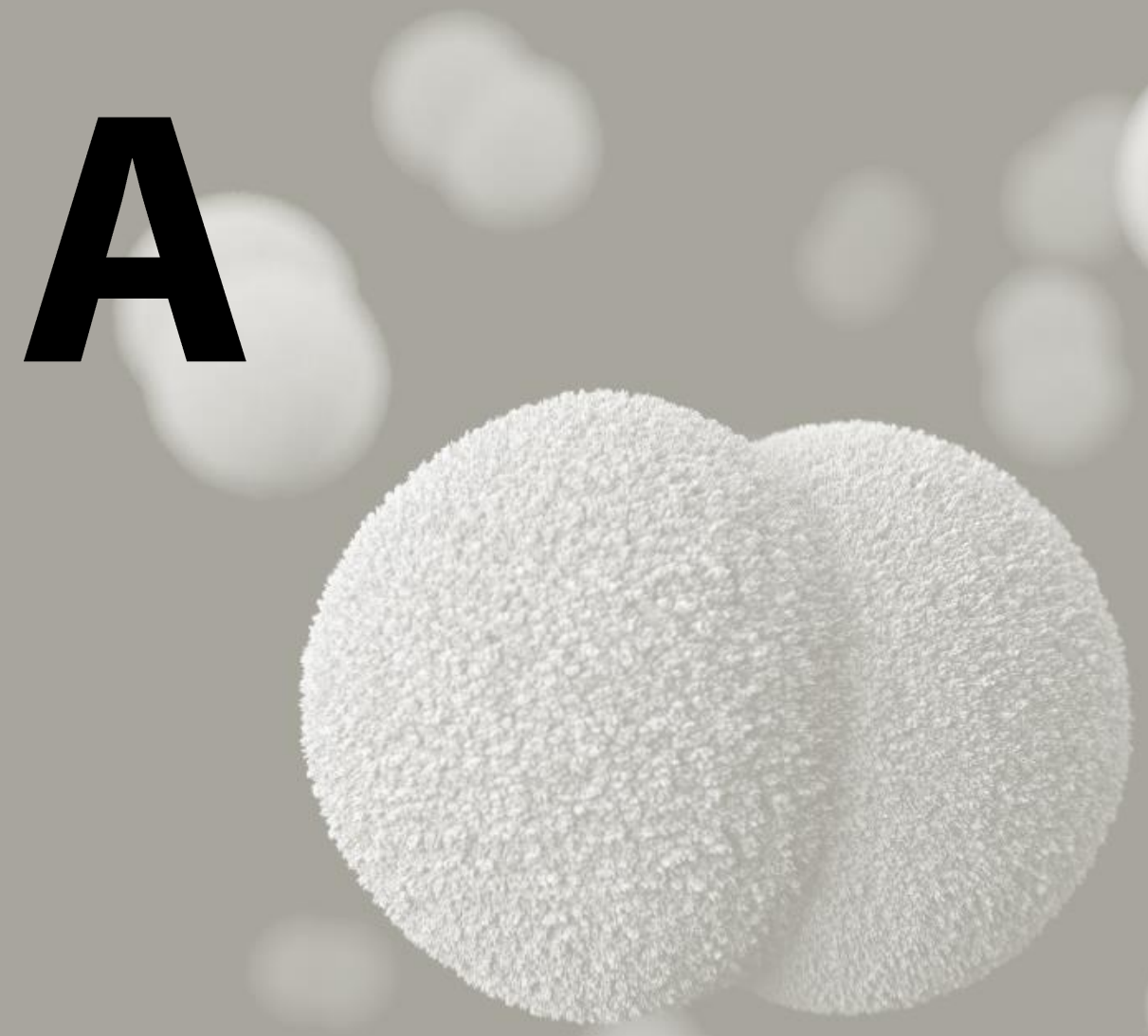




\section{Addendum}

Samenvatting en algemene discussie

Impact paragraph

List of publications

Curriculum vitae

Dankwoord 


\section{Samenvatting en algemene discussie}

Neisseria gonorrhoeae (NG) is een van de meest voorkomende bacteriële seksueel overdraagbare aandoeningen (SOA) wereldwijd. De introductie van nucleinezuur amplificatietests (NAATs) zorgde voor een revolutie waarop NG infecties gediagnosticeerd kunnen worden, vanwege de mogelijkheid om niet invasieve samples te gebruiken. Moderne NAAT's zijn zeer gevoelig en specifiek voor NG detectie en zijn geautomatiseerd. Hierdoor hebben NAAT's de NG kweek vervangen in routine screeningsdiagnostiek. De huidige NAAT's kunnen het antimicrobiële resistentie (AMR) profiel van een NG-isolaat echter niet bepalen. Aangezien NG resistentie heeft ontwikkeld tegen alle antibioticaklassen, is surveillance cruciaal om onbehandelbare NG te voorkomen. Huidige surveillance vereist een gekweekte NG isolaat om transmissie en AMR te kunnen monitoren. De kweek van NG is echter complex wat logistieke en technische expertise vereist, vanwege de veeleisende voedings- en omgevingsomstandigheden voor groei van NG. Ondanks de verbeterde diagnostiek en screeningsstrategieën blijft de incidentie van NG hoog. Daarom moet surveillance verbeterd worden en is het noodzakelijk om de transmissiedynamiek en AMR-acquisitie van NG beter te begrepen. Dit is met name belangrijk voor extragenitale infecties, vooral de orofarynx, aangezien deze worden beschouwd als een reservoir van AMR-genen voor NG. Dit komt doordat commensale Neisseria soorten vaak aanwezig zijn in de orofarynx en makkelijk DNA uitwisselen met NG.

De Wereldgezondheidsorganisatie (WHO) werd door verschillende meldingen van zeer resistente NG isolaten aangezet tot een zo gehete 'global action plan' om het ontstaan en verspreiding van resistente NG te voorkomen. De voorgestelde strategieën omvatten: effectieve diagnose en NG surveillance, systematische monitoring van behandelfalen, verbeteren van AMR surveillance, onderzoek naar nieuwe moleculaire surveillance methoden en nieuwe behandelingsopties. Met de onderzoeken in dit proefschrift wilden we meer kennis verwerven over effectieve diagnose, transmissie, verbeteren van surveillance en AMR beter te begrijpen bij NG. Hierbij bekeken we ook de potentiële centrale rol van de orofarynx en commensale Neisseria als reservoir voor resistentie. In dit hoofdstuk worden de belangrijkste conclusies van de studies in dit proefschrift 
besproken in perspectief van de bestaande literatuur. Daarnaast worden er aanbevelingen gedaan voor toekomstig onderzoek.

\section{De klinische relevantie van extragenitale NG infecties}

De noodzaak om extragenitaal te testen is erkend voor mannen die seks hebben met mannen (MSM), maar niet voor vrouwen en heteroseksuele mannen. Afgezien van MSM worden extragenitale testen alleen aanbevolen voor patiënten met een indicatie, zoals een voorgeschiedenis van directe blootstelling door anale en/of orale seks en/of symptomen. Dit is gelinkt aan het verschil in prevalentie van extragenitale NG bij MSM (3\% tot $21 \%)$ en heteroseksuele mannen en vrouwen ( $0 \%$ tot $3 \%)$. Recente epidemiologische studies tonen aan dat orofaryngeale NG veel voorkomt bij MSM en vrouwen. Wanneer er universeel routinematig werd getest, was de prevalentie van orofaryngeale NG 5,5\% bij MSM en 1,4\% bij vrouwen, waarbij de helft van de patiënten alleen een orofaryngeale infectie was. Een discussie rondom extragenitale NG infecties betreft de juistheid van de diagnose van deze infecties. Dit komt met name doordat de eerste generatie NAAT's vals-positieve resultaten konden geven door kruisreactiviteit met commensale Neisseria. Dit is opgelost in de momenteel gebruikte moderne NAAT's, maar deze zijn niet goedgekeurd voor extragenitale testen. Desondanks worden NAAT's aanbevolen voor de detectie van extragenitale NG mits het laboratorium specificaties voor prestatiekenmerken heeft opgesteld en deze overeenkomen met de huidige regelgeving. In eerdere studies is aangetoond dat de cobas 4800 CT/NG test zeer specifiek is en deze gebruikt kan worden voor anorectale swabs. Gebaseerd op deze resultaten veronderstellen wij dat de cobas 4800 CT/NG test ook goed zou presteren met orofaryngeale swabs. In hoofdstuk 2 hebben we de validiteit van de cobas 4800 CT/NG test voor detectie van C. trachomatis (CT) en NG in orofaryngeale swabs onderzocht. De cobas 4800 presteerde zeer goed om NG in orofaryngeale swabs te detecteren. De detectielimiet, gedefinieerd als $95 \%$ detectie, was 6,65×10² CFU/ml, maar belangrijker was de hoge specificiteit $(97,1 \%)$ en dat er geen kruisreactiviteit is waargenomen. Deze resultaten zijn in lijn met eerdere onderzoeken en tonen aan dat NG correct wordt gediagnosticeerd in extragenitale samples, waaronder de orofaryngeale swab. Echter, in onze studie wordt ook een potentieel belangrijk 
probleem gevonden, omdat er in theorie tot 25\% van de orofaryngeale NG infecties gemist zouden kunnen worden door een gemiddeld lage NG bacteriële load. Aangezien er geen aanwijzingen zijn dat de cobas 4800 CT/NG test ondermaats presteert ten opzichte van andere NAAT's, zou dit een veelvoorkomend probleem kunnen zijn, waarschijnlijk bij meerdere platformen. Vandaar dat er gevoeligere NAAT's ontwikkeld zouden moeten worden om gemiste orofaryngeale NG infecties te voorkomen die anders onopgemerkt zouden kunnen verspreiden.

De overdraagbaarheid van vaginale en extragenitale (anorectale en orofaryngeale) NG is nog niet geheel duidelijk. De load (hoeveelheid) van een pathogeen is gelinkt aan de overdraagbaarheid en klinische uitkomst bij andere SOA's. Vandaar dat NG bacteriële load gelinkt zou kunnen worden aan klinische uitkomst zoals symptomen. Voorgaande studies hebben een hogere NG load geobserveerd in urethrale infecties bij symptomatische mannen en anorectale infecties bij symptomatische MSM. Een lagere NG load werd waargenomen in orofaryngeale swabs van MSM ten opzichte van urethrale en anorectale swabs. Deze onderzoeken waren echter relatief klein en er is geen data over NG load bij vrouwen in relatie tot symptomen. Daarom is de NG load van genitale en extragenitale samples van een diverse populatie bepaald in hoofdstuk $\mathbf{3}$. In deze studie werden loads vergeleken tussen samplemateriaal en geslacht. Daarnaast werden er ook associaties tussen NG load en symptomen getest per seksuele risicogroep om een SOA te krijgen en sample materiaal. Uit onze data blijkt dat mannen en vrouwen een vergelijkbare NG load hebben op zowel genitale als extragenitale anatomische locaties. Dit impliceert dat een NG infectie even relevant zou zijn bij vrouwen als bij MSM. Onze data suggereren ook dat alle anatomische locaties een rol kunnen hebben in transmissie vanwege de aanzienlijke NG load. Wel zouden orofaryngeale NG infecties mogelijk een kleinere rol hebben gezien de lagere gemiddele NG load (16 tot 25-voud). Desondanks zijn orofaryngeale NG infecties zeer belangrijk, omdat het vaak enkele infecties betreft, merendeel asymptomatisch is en waarschijnlijk betrokken bij AMR ontwikkeling van NG. Daarom zou systematisch extragenitaal testen in routine diagnostiek de incidentie van NG kunnen verlagen. Echter, vanwege de kosten zal dit niet geïmplementeerd worden voor heteroseksuele mannen en vrouwen, omdat de 
prevalentie lager is. In lage-inkomenslanden zou dit zelfs nog minder toepasbaar zijn. In veel lage-inkomenslanden wordt een syndromale aanpak toegepast op basis van symptomen die gelinkt zijn aan een NG infectie. Omdat we in hoofdstuk 3 hebben aangetoond dat asymptomatische infecties voldoende load hebben voor transmissie, kunnen deze asymptomatische infecties meer schade veroorzaken. NG infecties zijn bijvoorbeeld gelinkt aan een verhoogd risico om geïnfecteerd te worden met HIV.

\section{De noodzaak om NG surveillance te verbeteren}

Ondanks verbeterde diagnostiek en preventie blijft de incidentie van NG infecties hoog. Enkele belangrijke populatiegroepen die risico lopen om geïnfecteerd te worden met NG zijn geïdentificeerd, zoals MSM, jongeren en sekswerkers. Vooral bij deze belangrijke populaties is surveillance essentieel om transmissie te stoppen en mogelijke uitbraken op te sporen. De huidige surveillance is afhankelijke van gekweekte isolaten om te kunnen genotypering waarmee genetische verwantschap kan worden vastgesteld. De kweek van NG mislukt echter vaak vanwege de veeleisende voedings- en omgevingsomstandigheden, wat leidt tot een laag percentage kweek bevestigde casussen. Daarom pleit de WHO in hun 'global action plan' om de kweekcapaciteit te vergroten en nieuwe moleculaire methoden voor surveillance te ontwikkelen. Hierdoor denken wij dat er behoefte is aan kweekonafhankelijke genotyperingsmethoden om het probleem met NG-kweek te vermeiden, waardoor de surveillance van NG wordt verbeterd. We hebben een kweekonafhankelijke methode ontwikkeld op basis van de veelgebruikte NG multi-antigen sequentietypering (NG-MAST) in hoofdstuk 4, genaamd "culture-free NG-MAST". Onze methode vertoonde geen kruisreactiviteit met niet-gonokokken Neisseria isolaten. Hierdoor kan de culture-free NG-MAST methode gebruikt worden om NG te genotyperen in klinische samples, inclusief extragenitale samples. Daarentegen kan de kweekafhankelijke NG-MAST niet worden gebruikt voor niet gekweekte extragenitale samples, omdat deze methode wel kruisreageert met commensale Neisseria, wat kan leiden tot verkeerde sequentietypen (ST). Bovendien heeft culture-free NG-MAST een hogere typeerbaarheid van $94 \%$ ten opzichte van de kweekafhankelijke methode met $62 \%$, in samples die ingezonden werden voor kweek. Doordat niet-kweekbare en extragenitale samples getypeerd kunnen worden in 
combinatie met de verhoogde typeerbaarheid zou de NG surveillance verbeterd kunnen worden. Wel moet men rekening houden dat (culture-free) NG-MAST vooral toepasbaar is om transmissieclusters te identificeren. Desondanks worden wel veel NG-MAST STs gekoppeld aan resistentie in NG, maar dit betekent niet perse dat de desbetreffende stam ook fenotypisch resistent is. Tot op heden blijft NG-kweek de 'gouden standaard' voor AMR-surveillance.

In de hoofdstukken 6 en 7 werd de potentiële meerwaarde van culture-free NG-MAST voor surveillance en uitbraakmanagement onderzocht. In hoofdstuk $\mathbf{6}$ zijn alle samples ( $n=196)$ die tussen januari 2017 en september 2018 routinematig gekweekt werden van onze SOA poli (GGD Zuid-Limburg), gegenotypeerd met culture-free NG-MAST. In totaal werden 152/196 (77\%) succesvol gegenotypeerd en van de 44 samples die mislukte, vertoonden er 11 een mogelijke gemixte infectie ( $\geq 2$ NG isolaten). Van de 196 samples waren er 115 kweekpositief, wat overeenkomt met een typeerbaarheid van $59 \%$. Dit is hoger dan het landelijk gemiddelde, waarschijnlijk doordat we alleen kweek adviseren bij een hoge bacteriële load. Desondanks heeft culture-free NG-MAST een hogere typeerbaarheid (77\% versus 59\%) en dit percentage is vergelijkbaar in kweekpositieve (80\%) versus kweeknegatieve samples (74\%). Wanneer we stratificeren op samplemateriaal was er geen verschil in typeerbaarheid van urinesamples tussen de methoden. Echter, zagen we een sterk verbeterde typeerbaarheid bij de andere samplematerialen wanneer culture-free NG-MAST werd gebruikt. In het bijzonder de orofaryngeale site, aangezien slechts $6,3 \%(1 / 16)$ kon worden getypeerd met kweek gebaseerde methoden, terwijl met culture-free NG-MAST 81\% (13/16) werd gegenotypeerd. Deze verbeterde typeerbaarheid bleek essentieel te zijn in een NGuitbraakonderzoek (hoofdstuk 7). Hierdoor identificeerde we een mogelijke uitbraak van NG in de jonge $(<25)$ heteroseksuele populatie welke onze SOA poli bezocht tussen oktober 2017 en maart 2019. We zagen een driemaal hoger aantal genitale NG infecties bij jonge heteroseksuelen per maand (4,5 per maand) ten opzichte van januari 2016 september 2017 (1,4 per maand, beschouwd als normale incidentie). Met behulp van culture-free NG-MAST werden drie onafhankelijke uitbraakclusters geïdentificeerd wat niet gedetecteerd zou zijn met kweek gebaseerde methoden. Ondanks de identificatie 
bleek implementatie van maatregelen om verdere verspreiding van NG te voorkomen moeilijk te zijn. Dit was te wijten aan beperkte informatie over seksuele partners en geen duidelijke associatie met een geografisch gebied (woonplaats). Het kan zijn dat de woonplaats geen goede voorspeller is, omdat de individuen in een cluster naar dezelfde school gaan of bijvoorbeeld dezelfde datingapp gebruiken. Eerdere studies tonen ook aan dat moleculaire epidemiologie, met behulp van NG-MAST, kan helpen bij het identificeren van grote clusters en nieuwe opkomende stammen, wat we ook hebben gezien in hoofdstukken 6 en 7. Daarom vinden wij dat culture-free NG-MAST een meerwaarde heeft voor NG surveillance, dit zal in meer detail toegelicht worden in de toekomst visie.

\section{De gevolgen van meerdere NG isolaten bij een patiënt}

Normaal gesproken wordt slechts één isolaat van een enkele anatomische locatie gekarakeriseerd in surveillance, waardoor NG infecties met meerdere isolaten, zoals gemixte infecties, over het hoofd worden gezien. Er zijn twee soorten infecties met meerdere NG isolaten; 1 ) verschillende stammen op afzonderlijke anatomische locaties, en 2) meerdere isolaten in één anatomische locatie (gemixte infectie). Beide typen infecties met meerdere NG isolaten werden waargenomen in eerdere onderzoeken, maar de frequentie van dit soort infecties varieert sterk tussen onderzoeken. Omdat veel van deze studies afhankelijk zijn van kweek, minder onderscheidende methoden gebruikten dan NG-MAST, of weinig voorkomende isolaten niet konden detecteren $(<10 \%$ in een mix), hebben we een targeted metagenomische methode ontwikkeld die weinig voorkomende isolaten kan detecteren en zeer onderscheidend is. We hebben deze methode gebruikt om meer inzicht te krijgen in de frequentie van meerdere NG isolaten bij een patiënt. Deze metagenomische methode is gebaseerd op onze zeer onderscheidende culture-free NG-MAST methode. In hoofdstuk 4 hebben we 81 MSM en 17 vrouwen met gepaarde NG positieve samples van afzonderlijke anatomische locaties geïncludeerd in onze studie. In totaal werden van 80/98 patiënten de gepaarde samples gegenotypeerd waarbij een kwart van de patiënten (20/80) geïnfecteerd waren met verschillende NG isolaten op afzonderlijke anatomische locaties op hetzelfde tijdstip. De geobserveerde proportie is hoger dan bij de meeste voorgaande 
onderzoeken mogelijk vanwege de omvang van het onderzoek, door de gebruikte meer discriminatoire methode en de inclusie van kweeknegatieve samples. Omdat het aandeel van verschillende NG-isolaten op afzonderlijke anatomische locaties hoog was, moest dit verder onderzocht worden om dit beter te begrijpen. De verschillende NGstammen op afzonderlijke anatomische plaatsen zouden het resultaat kunnen zijn van gemixte infecties, maar alleen de dominante stam van een anatomische locatie werd gekarakteriseerd in hoofdstuk 4. Onze hypothese is dat er ook additionele NGstammen, die voorheen niet werden ontdekt, aanwezig zouden kunnen zijn vanwege gemixte infecties. Daarom hebben we een vervolgstudie uitgevoerd in hoofdstuk $\mathbf{5}$ van de samples van MSM met verschillende NG-stammen op afzonderlijke anatomische locaties ( $\mathrm{n}=33$ ) van hoofdstuk 4. In hoofdstuk 5 werd een metagenomische genotyperingsmethode ontwikkeld door culture-free NG-MAST te combineren met next-generation sequencing en een analysepijplijn. Ondanks de geselecteerde MSM met verschillende NG-stammen op afzonderlijke anatomische locaties, laten de meeste samples (79\%, 26/33) een enkele NG-infectie zien. Onze studie levert additioneel bewijs dat gemixte NG-infecties voorkomen, maar waarschijnlijk bij een lage prevalentie. Mogelijke verklaringen voor het schijnbaar lage voorkomen van gemengde NG-infecties en de implicaties van meerdere NG-isolaten voor surveillance worden hieronder besproken.

Op basis van ons onderzoek in de hoofdstukken $\mathbf{4}$ en $\mathbf{5}$ blijkt dat gemixte NG-infecties minder vaak voorkomen dan verschillende NG-isolaten op afzonderlijke anatomische locaties. Er zijn verschillende verklaringen mogelijk zoals concurrentie tussen NGstammen, afzonderlijke introductie van NG en weefseltropisme. In een muisinfectiemodel werden fitheid en competitieve co-kweek van ceftriaxon-resistente NG-stammen bestudeerd door Vincent et al. (2018). De auteurs rapporteren significante verschillen in fitheid en in sommige competitieve co-kweken met wild-type NG werden geen ceftriaxon-resistente NG-bacteriën teruggevonden. Deze overgroei van een fittere, in dit geval de wild-type NG, nam in de loop van de tijd toe. Aangezien asymptomatische patiënten op zijn vroegst 14 dagen na mogelijke blootstelling worden getest, zoals beschreven in de Nederlandse SOA-richtlijnen, zou dit een deel van de lage 
frequentie van gemixte NG-infecties kunnen verklaren. De MSM in hoofdstuk 5 rapporteerden een mediaan van 10 (6-18, interkwartielbereik) sekspartners in de voorgaande zes maanden. Hierdoor is het mogelijk dat ze op verschillende tijdstippen aan NG zijn blootgesteld. Gecombineerd met competitie tussen NG-isolaten, zou een gevestigde NG-infectie van een nieuw geïntroduceerd NG-isolaat kunnen voorkomen. Het rapporteren van meerdere sekspartners weerspiegelt echter niet meerdere mogelijke blootstellingen van NG op een anatomische locatie. Uit onderzoek naar seksueel gedrag blijkt dat seksuele handelingen per sekspartner sterk kunnen variëren, inclusief condoomgebruik en soort seksuele handelingen per sekspartner. Ten slotte kan weefseltropisme bijdragen aan het lage voorkomen van gemixte infecties, aangezien sommige isolaten bepaalde anatomische plaatsen mogelijk slecht zouden kunnen infecteren. Echter ondergaan veel eiwitten fasevariatie die betrokken zijn bij de aanhechting van cellen. Dit resulteerd in adapatie om de adhesie aan verschillende menselijke cellen mogelijk te maken. Daarnaast kan NG zich aanpassen aan verschillende omgevingsomstandigheden door een reeks transcriptiefactoren. Hierdoor veronderstellen we dat NG-isolaten zich aan kunnen passen aan een anatomische locatie en is weefseltropisme waarschijnlijk geen verklaring voor de lage frequentie van gemixte infecties. Toch komen deze gemixte infecties voor en ze kunnen relevant zijn voor de behandeling, omdat een potentieel resistente NG-isolaat bij een gemixte infectie kan resulteren in behandelfalen. Daarnaast kunnen gemixte infecties relevant zijn voor de ontwikkeling van AMR, aangezien AMR-genen kunnen worden uitgewisseld tussen NG-isolaten. Zoals eerder besproken, wordt normaal gesproken één isolaat van een enkele anatomische locatie gekarakteriseerd in surveillance. Hierdoor zouden infecties met meerdere NG-isolaten worden gemist in de huidige surveillancestrategieën die potentieël de verspreiding van resistente NG-stammen mogelijk maken. Vandaar dat verschillende NG-isolaten op afzonderlijke anatomische locaties in de surveillance opgenomen zouden moeten worden, aangezien deze infecties vaak voorkomen. 


\section{De orofarynx als een reservoir voor antimicrobiële resistentie}

Mozaïekgenen kunnen voorkomen in het genoom van NG en leiden tot resistentie tegen verschillende antibiotica. Aangezien ceftriaxon de huidige eerstelijns empirische behandeling van NG is en er niet veel behandelingsopties over zijn, is AMR-onderzoek essentieel om onbehandelbare NG te voorkomen. Mozaïek penA-genen kunnen resulteren in ceftriaxonresistentie. Er wordt verondersteld dat deze mozaïek genen ontstaan door DNA-recombinatie met DNA van commensale Neisseria-soorten die doorgaans in de oropharynx voorkomen. Hierdoor wordt de orofarynx beschouwd als een reservoir van AMR-genen voor NG. In een studie van Igawa et al. (2018), is in vitro recombinatie van NG met $N$. cinerea DNA aangetoond. Dit weerspiegelt echter niet per definitie het proces in vivo, omdat bijvoorbeeld de regulatie van DNA-donatie onduidelijk is. Meestal zijn N. cinerea en N. subflava-groep betrokken bij mozaïek penAgenen in NG. Daarom zou de aanwezigheid van N. cinerea en N. subflava in de orofarynx tijdens een NG-infectie een voorwaarde zijn voor recombinatie. Om de aanwezigheid van Neisseria-soorten en mogelijke recombinatie in NG tijdens een orofaryngeale infectie te bestuderen, hebben we een metagenomische-methode ontwikkeld waarbij gebruik wordt gemaakt van nanopore-sequencing om amplicons van penA-genen en de flankerende regio's te karakteriseren (hoofdstuk 8). De flankerende regio's van het penA-gen werden gebruikt om de soort te kunnen identificeren. Hierdoor konden we penA-genen vergelijken en mogelijke recombinatiegebeurtenissen identificeren. In deze studie werden 64 NG-positieve orofaryngeale samples van MSM gekarakteriseerd, waarvan 61 penA PCR-positief waren. In bijna de helft van deze samples (28/61) werd een NG-contig waargenomen en 8/28 waren mozaïek. Op één na hadden alle mozaïek penA-genen aminozuursubstituties $1312 \mathrm{M}$ en V316T die gelinkt zijn aan ceftriaxonresistentie. In deze studie tonen we aan dat recombinatie tijdens een NGinfectie mogelijk zou kunnen zijn, omdat in alle samples (61/61) N. subflava en in meer dan de helft van de orofaryngeale samples (35/61) N. cinerea aanwezig waren. Bovendien was in alle samples met een mozaïek penA-gen in NG het mozaïekgedeelte van NG vergelijkbaar met $N$. cinerea of $N$. subflava. Hoewel de resistentie in Nederland laag is, werd bij $29 \%(8 / 28)$ van NG contigs een mozaïek penA waargenomen en dit is in 
lijn met de recente studie van de Laat et al. (2019). Recente epidemiologische studies in andere landen rapporteren het mozaïek penA-gen in 2,3\% tot $27 \%$ van de geteste isolaten. Zorgwekkend is dat de proportie stammen met mozaïek penA lijkt toe te nemen. Daarnaast worden A501-mutaties in niet-mozaïek penA vaak gerapporteerd die gelinkt zijn aan ceftriaxonresistentie. In onze studie van januari 2017 tot juni 2018 heeft slechts één NG-isolaat de mutatie A501V en is niet-mozaïek. Samengevat is het duidelijk dat commensale Neisseria-soorten die doorgaans voorkomen in de orofarynx ook tijdens een NG-infectie aanwezig zijn. Omdat Neisseria-soorten gemakkelijk DNA uitwisselen en het mozaïekgedeelte van NG vergelijkbaar was met commensale Neisseria-soorten, zou recombinatie in vivo een centrale rol kunnen spelen in de ontwikkeling van AMR in NG. Toch zijn er veel onbekende factoren, zoals de efficiëntie in DNA-donatie en opname, fitnesskosten van recombinatie, AMR in commensale Neisseria en mogelijke doding van NG door commensaal DNA.

\section{Afsluitende opmerkingen en toekomstperspectieven}

Ondanks vele inspanningen om NG-infecties op te sporen en te behandelen, blijft de incidentie hoog. Richtlijnen erkennen de noodzaak van extragenitale tests bij MSM en specifieke populaties. In dit proefschrift hebben we veel enkele orofaryngeale NGinfecties waargenomen die meestal asymptomatisch zijn. Hoewel de cobas 4800 CT/NGtest zeer goede prestaties vertoont om NG in orofaryngeale samples te detecteren, zou in theorie tot $25 \%$ kunnen worden gemist. Omdat er geen aanwijzingen zijn dat de cobas 4800 ondermaats presteert in vergelijking met andere NAAT's, zijn er gevoeligere testen nodig. Toekomstige studies moeten de impact van dit potentiële probleem bekijken. Herhaaldelijk testen van NG-negatieve orofarynx kan bijvoorbeeld worden uitgevoerd om de aanvankelijk gemiste infecties in te schatten.

Een van de doelen van dit proefschrift was om de surveillance van NG te verbeteren. We hebben aangetoond dat kweekonafhankelijke typering essentieel was om NGuitbraakclusters te identificeren. Zoals verschillende keren vermeld, zijn diagnostiek en surveillance de afgelopen decennia enorm verbeterd, maar NG blijft een wereldwijd probleem. Wij zijn van mening dat een prospectieve typering in combinatie met 
intensief bron en contact onderzoek zou kunnen helpen om NG-transmissie te beperken en uitbraken vroegtijdig op te sporen. Met deze gegevens zouden seksuele netwerken makkelijker geïdentificeerd kunnen worden om gerichte interventies zoals intensiever testen. Met onbeperkte middelen zouden idealiter alle NG-positieve samples moeten worden getypeerd om de hoogste resolutie te verkrijgen waarmee clusters geïdentificeerd kunnen worden. Samen met metadata van de GGD zou een interactieve monitoringtool voor NG-infecties voor clinici, epidemiologen en microbiologen kunnen worden gemaakt. In deze tool kan een waarschuwingssysteem worden geïmplementeerd om regio's of populaties te markeren die mogelijk extra aandacht nodig hebben. Zo kan bijvoorbeeld een mogelijke NG-uitbraak vroegtijdig worden geïdentificeerd om de transmissie proberen te stoppen. Helaas zijn de middelen beperkt en worden de kosten voor het typeren geschat op $€ 40$ bij gebruik van cultuur-free NGMAST, inclusief een analist. Een pilotstudie met de voorgestelde aanpak zou moeten worden uitgevoerd waar (hopelijk) clusters worden gezien om interventies mogelijk te maken wat uiteindelijk resulteerd in een afname van de incidentie van NG.

Gonorroe is tegenwoordig een grote uitdaging voor de volksgezondheid, niet alleen vanwege de hoge incidentie, maar ook vanwege de toenemende resistentie. Gebaseerd op eerdere resultaten en de resultaten in hoofdstuk 8 lijken commensale Neisseriasoorten een cruciale rol te spelen in de ontwikkeling van AMR van NG. De data over commensale Neisseria-soorten zijn echter beperkt. Daarom zou toekomstig onderzoek zich meer moeten richten op de commensale Neisseria dan alleen op NG. Zo is er weinig bekend over de minimale inhiberende concentratie (MIC) waarden bij deze soorten. Dit is met name relevant voor AMR in NG, omdat commensale Neisseria resistent zou kunnen worden na meerdere blootstellingen aan antibiotica en zouden dan AMR-genen kunnen uitwisselen met NG. Daarom is het belangrijk om de dynamiek van de commensale Neisseria beter te begrijpen. Zo zou een longitudinaal onderzoek waarbij individuen regelmatig worden gesampled, meer kennis op kunnen leveren. Daarbij wordt ingegaan op, maar niet beperkt tot, de samenstelling van de orofaryngeale Neisseria-soorten, mozaïekgenen, MIC-waarden en sequenties van het hele genoom van isolaten. 
Hoewel er een gebrek is aan nieuwe antibiotica voor de behandeling van NG, kunnen we de zorg verbeteren en het gebruik van ceftriaxon beperken om de ontwikkeling van AMR mogelijk vertragen. Door point-of-care-testen of nieuwe NAAT's te gebruiken die ook NG-isolaten kunnen identificeren die nog steeds behandeld kunnen worden met eerder gebruikte antibiotica zoals spectinomycine of ciprofloxacine. Hierdoor kan de patiënt behandeld worden met een van deze antibiotica in plaats van met ceftriaxon.

Samenvattend, met de introductie van NAATs werd de diagnose van een infectie met NG gevoeliger en met antibiotica werd de behandeling makkelijker. NG bleek echter een zeer adaptieve pathogeen te zijn die steeds resistenter wordt tegen alle antibiotica klassen. Daarom zijn er surveillanceprogramma's opgezet om resistente NG te monitoren en te voorkomen. Toch is er nog steeds veel onduidelijk, zoals de rol van commensale Neisseria en de aanhoudend hoge incidentie van NG wereldwijd. Het onderzoek dat in dit proefschrift wordt gepresenteerd, verduidelijkt een deel van de puzzel om de transmissiedynamiek te begrijpen, maar biedt ook tools om NGsurveillance te verbeteren. 


\section{Impact paragraph}

The World Health Organization estimates that 87 million new cases of Neisseria gonorrhoeae (NG) occur annually worldwide. An infection with NG can cause reproductive tract complications like pelvic inflammatory disease, infertility, ectopic pregnancy, and maternal death in women and urethritis and dysuria in men. Hence, preventing spread of sexually transmitted NG is a global health priority. Another major issue is that NG is increasingly resistant to all classes of antibiotics. Therefore, the main goals of this thesis were to improve surveillance, and to gain further knowledge on transmission dynamics and antimicrobial resistance acquisition in NG.

Bacterial load, the amount of bacteria, could be an indication for the transmissibility of a pathogen, in this case NG. We observed that all tested anatomical sites (genital, anorectal, and oropharynx) have sufficient bacterial load for transmission of NG. The observed bacterial loads were similar between men and women indicating that an infection with NG is equally relevant.

Current surveillance of NG characterizes only one isolate of one anatomical site, though many individuals can be infected concurrently with NG in separate anatomical sites. Studies presented in this thesis showed that in a quarter of patients distinct NG isolates were identified in separate anatomical sites. This would be missed with the current surveillance strategy and might have severe consequences. For example, this possibly allows dissemination of antimicrobial resistant (AMR) NG.

AMR is a global issue in NG that can lead to treatment failure and ultimately in untreatable NG. AMR can occur if DNA is exchanges by bacteria. In this exchange AMR genes can be donated by resistant NG isolates to susceptible ones. Another possibility is DNA donation by commensal Neisseria species that commonly reside in the oropharynx. A study in this thesis demonstrated that during all tested oropharyngeal infections with NG, commensal Neisseria were present. As these commensal Neisseria species readily exchange DNA with NG, the oropharynx is considered to be a reservoir for AMR development in NG. 
The studies presented in this thesis provide further knowledge on transmission dynamics and surveillance of NG. Some results can be readily applied to improve NG surveillance. Current surveillance of NG relies on culture that frequently fails because NG requires demanding nutritional and environmental conditions. In this thesis a surveillance method was developed that does not rely on culture. Studies presented in this thesis demonstrate the improved surveillance using our method and show that this method was essential to detect a potential outbreak of NG in young heterosexual in South Limburg, the Netherlands. The improved surveillance of this method could aid in unraveling of sexual networks to prevent transmission of NG. Potentially the improved detection of transmission and outbreaks might ultimately result in a lower incidence of $\mathrm{NG}$, thereby reducing sequelae and costs.

The results presented in this thesis provide insights for, but not limited to, microbiologists, epidemiologists, and policy makers. As discussed above, transmission dynamics of NG was investigated with the use of NG bacterial load. Surveillance methods were developed in this thesis and demonstrate the potential added value in surveillance. Also, the occurrence and impact of multiple distinct NG isolates in an individual is investigated in detail. Hence, our results can be used for modeling studies of transmission of NG and to improve surveillance guidelines.

Sharing study results and interdisciplinary collaborations helps to understand all aspects of a pathogen. Therefore, all studies presented in this thesis will be published in international journals, including datasets. Also, preliminary results were presented at national and international conferences to share knowledge and potentially start new collaborations. The studies presented in this thesis were performed in collaboration with local epidemiologists of the Public Health Service of South Limburg. Based on our results new studies are designed but also the culture-independent surveillance is being implemented. 
To conclude, this thesis provides further understanding of transmission dynamics and AMR acquisition of NG, and offers tools to improve surveillance. The cultureindependent surveillance method can be readily implemented in surveillance, thereby aiding in NG transmission prevention and outbreak detection. 



\section{List of publications}

In this thesis

Brian M. J. W. van der Veer, Petra F. G. Wolffs, Christian J. P. A. Hoebe, Juliën N. A. P. Wijers, Gènevieve A. F. S. Van Liere, Marita I. L. S. Werner, Amanja Verhaegh, Nicole H. T. M. Dukers-Muijrers, Lieke B. Alphen, Culture-independent genotyping revealed three strain clusters in a potential Neisseria gonorrhoeae outbreak in young heterosexuals (<25), the Netherlands, October 2017 to March 2019, Journal of Sexually Transmitted Diseases. 2021 Jan 12 Published ahead of print.

Julius M. Van Niekerk*, Brian M. J. W. van der Veer*, Christian J. P. A. Hoebe, Jeroen van de Bovenkamp, Christel van Herk, Inge H. M. van Loo, Lieke B. Alphen, Petra F. G. Wolffs, Despite Excellent Test Characteristics of the cobas 4800 CT/NG Assay, Detection of Oropharyngeal Chlamydia trachomatis and Neisseria gonorrhoeae Remains Challenging, Journal of Clinical Microbiology. 2021 Jan 21;58(2):e02137-20.

* contributed equally

Brian M. J. W. van der Veer, Christian J. P. A. Hoebe, Nicole H. T. M. Dukers-Muijrers, Lieke B. Alphen, Petra F. G. Wolffs, Men and Women Have Similar Neisseria gonorrhoeae Bacterial Loads: a Comparison of Three Anatomical Sites, Journal of Clinical Microbiology. 2020 Oct 21;58(11):e01171-20.

Brian M. J. W. van der Veer, Petra F. G. Wolffs, Christian J. P. A. Hoebe, Nicole H. T. M. Dukers-Muijrers, Lieke B. Alphen, Culture-free genotyping of Neisseria gonorrhoeae revealed distinct strains at different anatomical sites in a quarter of patients, the Netherlands, 2012 to 2016, Eurosurveillance. 2018 Dec;23(50).

Brian M. J. W. van der Veer, Petra F. G. Wolffs, Christian J. P. A. Hoebe, Nicole H. T. M. Dukers-Muijrers, Lieke B. Alphen, Metagenomic analysis shows distinct Neisseria gonorrhoeae strains at separate anatomical sites occur more commly than mixed strain infections: implications for surveillance, Submitted

Michiel H. C. Slaats, Brian M. J. W. van der Veer, Lieke B. Alphen, Christian J. P. A. Hoebe, Nicole H. T. M. Dukers-Muijrers, Petra F. G. Wolffs, Culture-free genotyping improves surveillance of Neisseria gonorrhoeae, especially in oropharyngeal samples, the Netherlands, 2017 to 2018, Submitted 
Brian M. J. W. van der Veer, Christian J. P. A. Hoebe, Nicole H. T. M. Dukers-Muijrers, Lieke B. Alphen, Petra F. G. Wolffs, Despite no ceftriaxone resistance in the Netherlands many Neisseria gonorrhoeae isolates have a mosaic penA gene, Manuscript in preparation

\section{Additional publications}

Koen M.F. Gorgels, Jozef Dingemans, Brian M. J. W. van der Veer, Volker Hackert, Audrey Y.J. Hensels, Casper D.J. Den Heijer, Lieke B. van Alphen, Paul H.M. Savelkoul Christian J.P.A. Hoebe, Linked nosocomial outbreak of COVID-19 in three facilities for people with intellectual and developmental disabilities due to SARS-CoV-2 variant B1.1.519 with spike mutation T478K in the Netherlands, Submitted

Michiel H. C. Slaats, Inge H. M. van Loo, Astrid M. L. Oude Lashof, Brian M. J. W. van der Veer, Limmie Y. L. Liu, Rein Posthuma, Suzanne van Santen, Fabienne Magdelijns, Antibody response of hospitalized patients with COVID-19, a follow-up study, Submitted

Liteboho D. Maduna, Marleen M. Kock, Brian M. J. W. van der Veer, Oscar Radebe, James Mclntyre, Lieke B. van Alphen, Remco P. H. Peters, Antimicrobial Resistance of Neisseria gonorrhoeae Isolates from High-Risk Men in Johannesburg, South Africa, Antimicrobial Agents and Chemotherapy. 2020 Oct 20; 64(11):e00906-20.

Shih-Chin Cheng, Jessica Quintin, Robert A. Cramer, Kelly M. Shepardson, Sadia Saeed, Vinod Kumar, Evangelos J Giamarellos-Bourboulis, Joost H.A. Martens, Nagesha Appukudige Rao, Ali Aghajanirefah, Ganesh R. Manjeri, Yang Li, Daniela C. Ifrim, Rob J.W. Arts, Brian M.J.W. van der Veer, Peter M.T. Deen, Colin Logie, Luke A. O'Neill, Peter Willems, Frank L. van de Veerdonk, Jos W.M. van der Meer, Aylwin Ng, Leo A.B. Joosten, Cisca Wijmenga, Hendrik G. Stunnenberg, Ramnik J. Xavier, and Mihai G. Netea, mTOR- and HIF-1a-mediated aerobic glycolysis as metabolic basis for trained immunity, Science. 2014 Sep 26;345(6204):1250684. 


\section{Curriculum vitae}

Brian van der Veer werd geboren op 15 juni 1991 te Boxmeer. Hij voltooide de HAVO in 2008 aan de scholengemeenschap Stevensbeek. Daarna werd de Bachelor opleiding 'Hoger Laboratorium Opleiding Biochemie' aan Hogeschool van Arnhem en Nijmegen succesvol afgerond in 2013, hierbij verkreeg hij de titel Bachelor of Applied Sciences. De titel Bachelor of Science werd in hetzelfde jaar verkregen aan de Radboud Universiteit. Hierop volgde de research Master 'Medical Biology' aan de Radboud Universiteit waar de titel Master of Science bene meritum werd behaald in 2015. Zijn promotieonderzoek ging van start in juli 2016 bij de afdeling Medische Microbiologie van het Maastricht Universitair Medisch Centrum+ (MUMC+) in samenwerking met GGD Zuid Limburg, onder leiding van Prof. dr. Christian Hoebe, Dr. Lieke van Alphen en Dr. Petra Wolffs. Per november 2020 is hij werkzaam als bioinformaticus bij dezelfde afdeling van het MUMC+, waar hij onderzoek verricht naar uitbraken en het voorkomen van (nieuwe) varianten van SARS-CoV-2. 



\section{Dankwoord}

$\mathrm{Na}$ veel te hebben geleerd en geschreven ben ik aangekomen bij de laatste zinnen van mijn proefschrift. De afgelopen jaren heb ik als zeer leerzaam, intensief en soms pittig ervaren. Tijdens mijn promotieonderzoek heb ik met veel mensen fijn samengewerkt wat ongetwijfelt dit proefschrift beter heeft gemaakt, hiervoor wil ik jullie graag bedanken.

Als eerste wil ik graag mijn promotieteam bedanken: Prof. dr. Christian Hoebe, Dr. Lieke van Alphen en Dr. ir. Petra Wolffs. Ontzettend bedankt voor de kans die jullie mij hebben gegeven met dit promotietraject. Ik heb heel veel van jullie kunnen leren door jullie diverse expertises en enthousiasme. Door de vele en soms pittige discussie en mij, zoals Petra altijd zegt, "onder te dompelen in SOA's" hebben jullie mij klaargestoomd tot onderzoeker. Ondanks jullie drukke agenda's kon ik rekenen op jullie goede feedback. Dit was zeker in de laatste maanden erg uitdagend. Naast onderzoek hebben we ook leuke congressen gehad naar Malta en Canada. Daar plande we toch altijd op zijn minst 1 dag in om de omgeving en stad te verkennen. De NVMM was ook altijd vaste prik waar je op de feestavond niet onder een dansje uitkwam. Lieke en Petra zijn dan ook als één van de eerste te vinden op dansvloer, waar wij als promovendi toch vaak eerst een paar biertjes voor nodig hebben.

De leden van de beoordelingscommissie: Prof. dr. Paul Savelkoul, Prof. dr. Jochen Cals, Dr. Sylvia Bruisten en Dr. Janneke Heijne, hartelijk dank voor het beoordelen van dit proefschrift. Daarnaast wil ik ook alle leden van de corona (beetje gek om dit in deze tijd te zeggen) bedanken voor jullie tijd en deelname aan de oppositie.

Ook wil ik mijn paranimfen bedanken. Gianluca, I would like to thank you for the many spontaneous and sometimes philosophical talks also in difficult times. I appreciate your honesty and your hospitality. You invited us many times for diner where you shared your cooking tips. Ivon and I loved our trip to Sicily where you showed us around and had nice food and drinks. Mayk, bedankt voor alle hulp in het lab waar we sinds kort het 
leuke maar soms ook frustrerende onderzoek naar Neisseria delen. Jij bent naast een goede analist ook super creatief wat onder andere te zien is aan de cover van dit proefschrift. Daarnaast konden we elkaar ook altijd helpen met tips voor vanallerlij klusjes in en rondom het huis.

Alle collega's van de afdeling Medische Microbiologie bedankt voor de fijne werksfeer en leuke afdelingsuitjes en borrels. Erik en Christel, voor vragen in het lab en sparren om de methodes net iets beter te krijgen. Zonder ons wonder polymerase "AccuPrime" waren veel van mijn studies niet zo goed gelukt. Natuurlijk ook medeauteurs Amanja, Christel, Gènevieve, Inge, Jeroen, Juliën, Julius, Nicole, Marita en Michiel voor jullie kritische blik en bijdrage aan de verschillende publicaties. Daarnaast wil ik de SOA onderzoeksgroep bedanken voor de vele ideeën en inzichten om het gonorroe onderzoek te sturen. I would also like to thank the microbiome and antimicrobial resistance research group for the technical discussions and combining insights of their field with my STI research. My fellow researchers Birke, Carla, Casper, Charlotte, Chris, Christel, David, Erik, Giang, Gianluca, Heike, Judith, Julius, Kevin, Lars, Liene, Matthew, Mayk, Melissa, Nader, Tessa, Wesley for your help but also for the nice work environment, coffee-breaks, drinks and BBQs. Kevin, zoals jij in je boekje ook al schreef zijn wij als een co-infectie van chlamydia en gonorroe. Wij versterkte elkaar in onderzoek, maar ook tijdens de pauzes met sterke verhalen en onzin. My roomies Gianluca, Liene and Niels, we had a great time making jokes, talking about trips, complain about coffee but also help each other with research and personal problems like a broken car.

Ook wil ik de collega's van de Moleculaire Diagnostiek bedanken voor het meedenken en jullie flexibiliteit, zodat ik van jullie apparatuur gebruik kon maken. Daarnaast wil ik Peter Terporten bedanken voor het aanleveren van veel data en het leren van SQL, zodat ik zelf de queries kon doen om de data te verzamelen. Dit heeft mij ontzettend veel tijd bespaard tijdens mijn onderzoek. De mede onderzoekers van de afdeling Seksuele Gezondheid van de GGD wil ik ook graag bedanken. Het was fijn en leerzaam om 
onderdeel te zijn van ons multidisciplinair onderzoeksteam. Dankzij deze samenwerking worden onze studies naar een hoger niveau getrokken. In het bijzonder wil ik Gènevieve, Juliën, Nicole en Ymke bedanken voor het aanleveren van ontzettend veel data, goede feedback en hulp bij de statistische analyses.

Mijn familie en vrienden wil ik bedanken voor de interesse en zorgen over mijn onderzoek en de lange ritten naar Maastricht, ondanks dat het nog altijd lastig te begrijpen is wat ik nu precies doe. Ik kon op jullie rekenen voor feestjes en bordspellen wat hielp om af te schakelen, zodat ik weer fris kon gaan werken. Ivon, ik wil jou bedanken dat je dit mogelijk hebt gemaakt voor mij, want als ik weer eens heel laat thuis was stond het eten klaar en had je zelfs op me gewacht. Daarnaast had je soms al het hele huis gepoetst ook al mocht je dat niet van mij, maar hierdoor kon ik wel focussen op mijn onderzoek. Ook's avonds en in de weekenden hielp je me als ik doorwerkte met een kop koffie en wat lekkers. Als het toch echt tijd was om te stoppen kwam je met een speciaal biertje aan om een bordspel te doen of wat op Netflix te kijken. Jij werkt ook in het lab dus snapte nog wel iets van wat ik aan het doen was. Vooral de tegenslagen en frustraties in het lab wanneer iets niet werkt zoals je verwacht kunnen we altijd delen met elkaar. Dennis, ik kan me nog goed herinneren dat je mij van mijn scriptie af haalde om nou toch echt eens te ontspannen, want je zag dat ik niet meer vooruit kwam en gefrustreerd werd. Dit advies heb ik hierna nog vaak opgevolgd door toch even te gamen of met jou een biertje te drinken. Pap en mam, bedankt voor alle kansen en steun die jullie ons hebben gegeven. Onbezorgt heb ik mijn studies kunnen doen en tijdens mijn promotieonderzoek vroegen jullie ook vaak hoe het ging. Soms probeerde ons pap, ja ben een Brabander, te begrijpen wat ik deed en probeerde zelfs mijn eerste artikel te lezen. Je zei dan dat je het snapte, maar ik prikte daar al snel doorheen en vroeg dan door. Je moest dan toch toegeven dat je het eigenlijk niet helemaal begreep. Kortom, dat ik dit allemaal heb kunnen bereiken heb ik aan jullie te danken. 